\section{PRISM (Polarized Radiation Imaging and Spectroscopy Mission): an extended white paper}

To cite this article: Philippe André et al JCAP02(2014)006

View the article online for updates and enhancements.

\section{Related content}

- Gravitational-wave mission selected for
$\frac{\text { launch }}{\text { Sarah Tesh }}$
- $\frac{\text { How well can future CMB missions }}{\text { constrain cosmic inflation? }}$
Jérôme Martin, Christophe Ringeval and
Vincent Vennin
- Orthogonal non-Gaussianity in DBI
galileon: prospect for Planck polarization
and post-Planck experiments
Kazuya Koyama, Guido Walter Pettinari,
Shuntaro Mizuno et al.

\section{Recent citations}

- Primordial black holes as dark matter and
$\frac{\text { gravitational waves from bumpy axion }}{\text { inflation }}$
Ogan Özsoy and Zygmunt Lalak
- Can dark neutrino interactions phase out
$\frac{\text { the Hubble tension? }}{\text { Subhajit Ghosh et al }}$
- Imprints of axion superradiance in the
$\frac{\text { CMB }}{\text { Diego Blas and Samuel J. Witte }}$




\title{
PRISM (Polarized Radiation Imaging and Spectroscopy Mission): an extended white paper
}

\section{The PRISM collaboration ${ }^{1}$}

E-mail: naselsky@nbi.dk

Received January 17, 2014

Accepted January 17, 2014

Published February 5, 2014

\begin{abstract}
PRISM (Polarized Radiation Imaging and Spectroscopy Mission) was proposed to ESA in May 2013 as a large-class mission for investigating within the framework of the ESA Cosmic Vision program a set of important scientific questions that require high resolution, high sensitivity, full-sky observations of the sky emission at wavelengths ranging from millimeter-wave to the far-infrared. PRISM's main objective is to explore the distant universe, probing cosmic history from very early times until now as well as the structures, distribution of matter, and velocity flows throughout our Hubble volume. PRISM will survey the full sky in a large number of frequency bands in both intensity and polarization and will measure the absolute spectrum of sky emission more than three orders of magnitude better than $C O B E$ FIRAS. The data obtained will allow us to precisely measure the absolute sky brightness and polarization of all the components of the sky emission in the observed frequency range, separating the primordial and extragalactic components cleanly from the galactic and zodiacal light emissions. The aim of this Extended White Paper is to provide a more detailed overview of the highlights of the new science that will be made possible by PRISM, which include: (1) the ultimate galaxy cluster survey using the Sunyaev-Zeldovich (SZ) effect, detecting approximately $10^{6}$ clusters extending to large redshift, including a characterization of the gas temperature of the brightest ones (through the relativistic corrections to the classic SZ template) as well as a peculiar velocity survey using the kinetic SZ effect that comprises our entire Hubble volume; (2) a detailed characterization of the properties and evolution of dusty galaxies, where the most of the star formation in the universe took place, the faintest population of which constitute the diffuse CIB (Cosmic Infrared Background); (3) a characterization of the $\mathrm{B}$ modes from primordial gravity waves generated during inflation and from gravitational lensing, as well as the ultimate search for primordial non-Gaussianity using CMB polarization, which is less contaminated by foregrounds on small scales than the

\footnotetext{
${ }^{1}$ www.prism-mission.org. This is an extended version of the White paper submitted to ESA in June 2013 (large) missions as part of the ESA Cosmic Vision Programme. The approximate launch dates announced for these two slots, known as L3 and L4, are 2028 and 2034, respectively.
} (astro-ph arXiv:1306.2259) as part of a competition to select the science themes for the next two ESA L-class
\end{abstract}


temperature anisotropies; (4) a search for distortions from a perfect blackbody spectrum, which include some nearly certain signals and others that are more speculative but more informative; and (5) a study of the role of the magnetic field in star formation and its interaction with other components of the interstellar medium of our Galaxy. These are but a few of the highlights presented here along with a description of the proposed instrument.

Keywords: CMBR detectors, CMBR experiments, cosmological parameters from CMBR 


\section{Contents}

1 Executive summary 1

1.1 Ultimate galaxy cluster survey and census of structures in our Hubble volume 2

1.2 Understanding the Cosmic Infrared Background and star formation across cosmic time

1.3 Detecting inflationary gravity waves and constraining inflationary scenarios $\quad 3$

1.4 Probing new physics through CMB spectral distortions

1.5 Probing Galactic astrophysics

\section{Legacy archive}

3 Probing the Universe with galaxy clusters

3.1 Characterizing the PRISM cluster catalog

3.2 Thermal SZ maps

3.3 Cluster counts as a cosmological probe

3.4 Cosmic velocity field

3.5 Patchy reionization

3.6 Relativistic and non-thermal effects

3.7 Diffuse SZ and the cosmic web

3.8 Polarized SZ effect

Extragalactic sources and the cosmic infrared background

4.1 Early evolution of galaxies

4.2 The cosmic infrared background

4.3 Radio sources

4.4 Extragalactic sources and the integrated Sachs-Wolfe effect

5 Inflation and CMB primordial B-modes

6 CMB at high resolution

6.1 Probing the dark universe with CMB lensing 21

6.2 Primordial non-Gaussianity 23

6.3 Parameters from high-resolution polarization spectra 26

7 CMB spectral distortions

$\begin{array}{lll}7.1 & \text { Reionization and structure formation } & 28\end{array}$

$\begin{array}{ll}7.2 & \text { Constraining the inflaton } \\ 7.3 & \text { Decaying }\end{array}$

$\begin{array}{lll}7.3 & \text { Decaying and annihilating relics } & 30\end{array}$

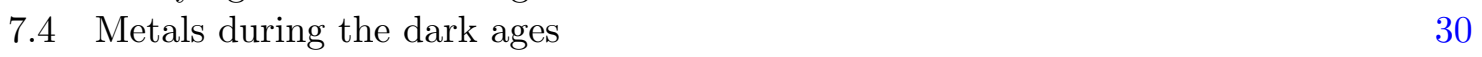

7.5 Cosmological recombination radiation 32

$\begin{array}{lll}7.6 & \text { Hydrogen line scattering } & 32\end{array}$

$\begin{array}{lll}7.7 & \text { Rayleigh scattering } & 33\end{array}$

7.8 Cooling of matter 33

7.9 Cosmic strings and primordial black holes $\quad 34$

7.10 PRISM's discovery potential using spectral distortion 34 
8 Structure of the dusty magnetized Galactic ISM $\quad 34$

8.1 Structure of interstellar medium 34

8.2 Galactic magnetic field and star formation 35

8.3 Nature of interstellar dust 36

9 Zodiacal light emission $\quad 36$

$\begin{array}{ll}10 \text { Strawman mission concept } & 37\end{array}$

10.1 Instruments 38

10.1.1 The polarimetric imager 38

10.1.2 The absolute spectrophotometer $\quad 40$

10.1.3 Synergy of the two instruments 41

10.2 Experimental challenges 41

10.2.1 Telescope 41

10.2.2 Cooling chain 43

10.2.3 Scan strategy 43

10.2.4 Polarization modulation 44

10.2.5 Detectors 44

10.2.6 Detector time constants 45

10.3 Ancillary spacecraft 45

11 Competition and complementarity with other observations 46

11.1 Ground-based and balloon-borne CMB B-mode experiments 46

11.2 Other space CMB projects 47

11.3 Galaxy clusters observations 48

11.4 Other sub-millimeter and far-infrared initiatives 49

$\begin{array}{ll}11.5 & \text { Other dark energy probes } \\ 11.6\end{array}$

$\begin{array}{ll}11.6 \text { SPICA } & 51\end{array}$

11.7 Square Kilometer Array (SKA) 51

$\begin{array}{ll}\text { The PRISM collaboration } & 65\end{array}$

The PRISM Science Case and White Paper Coordination 68

$\begin{array}{lc}\text { The PRISM Steering Committee } & 68\end{array}$

\section{Executive summary}

The Polarized Radiation Imaging and Spectroscopy Mission (PRISM) is a proposed largeclass mission designed to carry out the ultimate survey of the microwave to far-infrared sky in both intensity and polarization as well as to measure its absolute emission spectrum. PRISM will consist of two instruments:

1. A high angular resolution polarimetric imager with a $3.5 \mathrm{~m}$ usable diameter telescope, cooled to below $10 \mathrm{~K}$ in order to maximally reduce the photon noise due to the thermal emission of the mirrors. This instrument will map the intensity and polarization of the complete sky in 32 broad frequency bands between $30 \mathrm{GHz}(1 \mathrm{~cm})$ and $6 \mathrm{THz}(50$ microns) with unprecedented sensitivity and with an angular resolution ranging from about 17 arcminutes to about 6 arcseconds. 
2. A lower angular resolution $\left(1.4^{\circ}\right)$ spectrometer that will compare the sky frequency spectrum to a nearly perfect reference blackbody and measure the absolute sky emission over the same frequency range.

The data from these two instruments will enable PRISM to carry out breakthrough science by answering key questions in many diverse areas of astrophysics and fundamental science as well as providing the astronomical community with high quality, full sky observations of emission over a large frequency range.

Highlights of the new science from PRISM include:

\subsection{Ultimate galaxy cluster survey and census of structures in our Hubble volume}

Because of the tight correlation between integrated $y$-distortion and cluster mass as well as the redshift independence of the surface brightness, the Sunyaev-Zeldovich (SZ) effect offers the best method for assembling a catalog of clusters at high redshift to be used for cosmology. When PRISM is launched (foreseen for 2028 or later), all-sky cluster samples (e.g., from eROSITA, Euclid) will likely comprise some $10^{5}$ objects, mostly at $z<1$. PRISM will find 10 times more clusters, which importantly will extend to deeper redshifts, with many thousands beyond $z=2$. In fact, PRISM will detect all clusters of mass larger than $5 \times 10^{13} M_{\odot}$ in the universe. Owing to its broad spectral coverage, high angular resolution, and exquisite sensitivity, using the kinetic SZ effect, PRISM will measure the peculiar velocity of hundreds of thousands of clusters. PRISM thus will carry out a complete survey of the large-scale velocity field throughout our entire Hubble volume. PRISM will also measure the gas temperature of more than ten thousand massive galaxy clusters using the relativistic corrections to the classic SZ spectral distortion spectrum.

By cross-correlating cluster detections with measurements of gravitational lensing of the CMB, PRISM will measure cluster masses and calibrate the scaling laws crucial for cosmological studies. This will be possible not only for PRISM clusters, but for all cluster samples including X-ray and optically selected clusters, and even candidate high redshift proto-clusters detected in the far infrared. The PRISM cluster catalogue will provide an exceptionally powerful probe of dark energy, modified gravity, and structure formation throughout the Hubble volume.

PRISM will provide a comprehensive view of both the dark matter and the baryonic matter distributions, as well as their relation and its evolution over cosmic time. With ultra-precise measurement of gravitational lensing of the $\mathrm{CMB}$, maps of the dark matter distribution will be obtained, which can be correlated with various baryonic tracers. These include the hot gas observed through the thermal SZ effect, optical and IR galaxies, extragalactic radio sources catalogues, and the cosmic infrared background (CIB) arising from primordial galaxies reaching redshifts $z>5$. Cross-correlations with upcoming cosmic shear surveys such as Euclid will also be extremely interesting. In this way, invaluable insight into the transformation of baryons from primordial gas into stars within their dark matter halo hosts will be obtained as well as a multi-component view of the distribution of mass and its tracers in the entire observable universe. Because the CMB is the most distant source plane, PRISM maps the density contrasts at much higher redshifts than traditional gravitational lensing surveys. 


\subsection{Understanding the Cosmic Infrared Background and star formation across cosmic time}

Most star formation in the universe took place at high redshift. Hidden from optical observations by shrouds of dust in distant galaxies, star formation at large redshift is visible in the far infrared. Emission from these dusty galaxies constitutes the cosmic infrared background (CIB), which PRISM owing to its high sensitivity and angular resolution in the far infrared is uniquely situated to investigate. The survey will sharpen and extend to higher redshifts the determination of the bolometric luminosity function and the clustering properties of starforming galaxies. Tens of thousands of easily recognizable, bright, strongly lensed galaxies and hundreds of the very rare maximum starburst galaxies beyond $z>6$ will be detected, providing unique information on the history of star formation, the physics of the interstellar medium under a variety of conditions including the most extreme, and the growth of large-scale structure, including proto-clusters of star-forming galaxies. The survey will also probe the evolution of radio sources at (sub-)mm wavelengths and provide measurements of the spectral energy distribution (SED) of many thousands of radio sources over a poorly explored but crucial frequency range.

The broad frequency coverage and many frequency bands enable us to model the SEDs of individual distant sources as a superposition of emission from enshrouded star formation and emission powered by the active nucleus. PRISM will thus provide insight into the interplay between these two components and their claimed co-evolution. The measurement of the source SEDs provide photometric redshifts, enabling optimized follow-up with high resolution ground-based spectroscopic observations.

In addition, the many frequency bands of PRISM will enable us to decompose the CIB of unresolved galaxies into sub-components originating from different redshift shells and thus constrain models of structure and star formation throughout cosmic history. By measuring both the total absolute CIB emission and its fluctuations over the CIB emission spectrum bump as well as resolving many individual high redshift galaxies, PRISM will provide a comprehensive view of the far infrared background.

\subsection{Detecting inflationary gravity waves and constraining inflationary scenarios}

Present precision measurements of cosmic microwave background (CMB) temperature anisotropies lend considerable support to simple models of inflation. However the most spectacular prediction of inflation - the generation of gravitational waves with wavelengths as large as our present horizon - remains unconfirmed. Several initiatives from the ground and from stratospheric balloons are currently underway to attempt to detect these gravitational waves through the B-mode spectrum of the CMB polarization. However these initiatives suffer severe handicaps including limited frequency coverage due to atmospheric opacity and emission, unstable seeing conditions, and far sidelobe pickup of ground emission. Only from space can one reliably detect the very low- $\ell$ B-modes due to the re-ionization bump. Because of its broad frequency coverage and extreme stability, PRISM will be able to detect B-modes at $5 \sigma$ for $r=5 \times 10^{-4}$, even under pessimistic assumptions concerning the complexity of the astrophysical foreground emissions that must be reliably removed. Moreover, PRISM will be able to separate and filter out the majority of the lensing signal due to gravitational deflections. Hence PRISM will perform the best possible measurement of primordial CMB B-mode polarization, and hence the best possible measurement of the corresponding inflationary tensor perturbations achievable through CMB observations. 


\subsection{Probing new physics through CMB spectral distortions}

The excellent agreement between the microwave sky emission and the perfect blackbody observed by the COBE FIRAS instrument is rightfully highlighted as a crucial confirmation of Big Bang cosmology. However theory predicts that at higher sensitivity this agreement breaks down. Energy injection at any redshift $z \lesssim($ few $) \times 10^{6}$ superimposes spectral distortions relative to a perfect CMB blackbody spectrum. Some of the predicted deviations are nearly sure bets. Others provide powerful probes of possible new physics. The PRISM absolute spectrometer will measure the spectrum more than three orders of magnitude better than FIRAS. $y$-distortions from the re-ionized gas as well as from hot clusters constitute a certain detection. However $\mu$-distortions and more general spectral distortions have the potential to uncover decaying dark matter and to probe the primordial power spectrum on very small scales that cannot be measured by other means, being contaminated by the nonlinearity of gravitational clustering at late times.

\subsection{Probing Galactic astrophysics}

PRISM will have a major impact on Galactic astrophysics by providing a unique set of all-sky maps. The PRISM data will extend Herschel dust observations to the whole sky, map the absolute sky intensity and polarized emission with high sensitivity in more than 20 different frequency bands, and also map emission lines key to quantifying physical processes in the interstellar medium. The survey will have the sensitivity and angular resolution required to map dust polarization down to sub-arcminute resolution even at the Galactic poles. No other project provides a comparable perspective on interstellar components over such a wide range of scales.

The PRISM data will provide unique clues to study the interstellar medium, the Galactic magnetic field, and star formation, and will address three fundamental questions in Galactic astrophysics: what are the processes that structure the interstellar medium? What role does the magnetic field play in star formation? What are the processes that determine the composition and evolution of interstellar dust?

\section{Legacy archive}

The science themes outlined above sample but a few of the highlights of the rich and diverse physics and astrophysics that PRISM will be able to carry out. These are the main drivers for the mission definition. However a secondary but also important objective is the collection of high quality survey full-sky data for a large variety of applications by the astronomical community.

The hundreds of intensity and polarization maps of PRISM spanning over two decades in frequency will constitute a legacy archive useful for almost all branches of astronomy for decades to come. Combining low resolution spectrometer data and high resolution images from the imager, PRISM will deliver a well calibrated and fully characterized spectropolarimetric survey of the full sky from $50 \mu \mathrm{m}$ to $1 \mathrm{~cm}$. The spectral resolution will range from about $0.5 \mathrm{GHz}$ to $15 \mathrm{GHz}$ at $1.4^{\circ}$ angular resolution, and from $\delta \nu / \nu \approx 0.025$ to 0.25 at the diffraction limit of a $3.5 \mathrm{~m}$ telescope (from $\sim 6^{\prime \prime}$ to $17^{\prime}$ ). It is not possible to foresee all the ways in which this extraordinary data set can be exploited.

The PRISM collaboration will make public full-sky maps of the absolute emission of the sky (in intensity and polarization) in many frequency channels, as well as maps of absolute temperature of the CMB and its polarization (at a resolution of about 2 arcminutes with a 

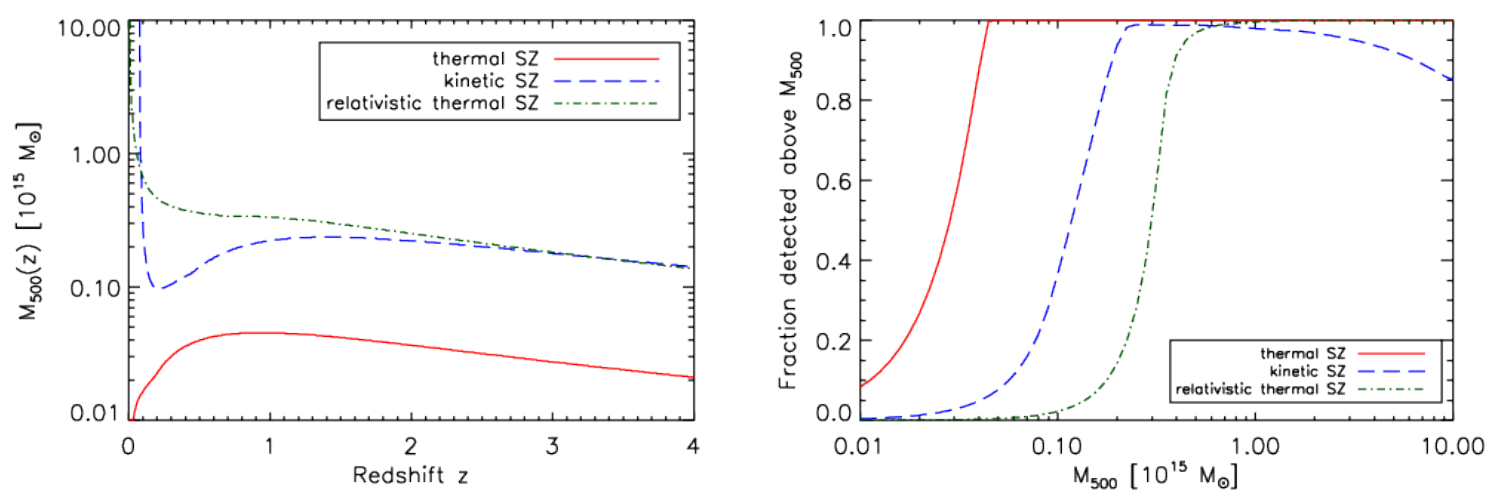

Figure 1. Left panel: lower mass limits for detection of the labeled SZ effects at signal-to-noise $S / N>5$ as a function of redshift. Right panel: the completeness of the detection for all three effects, as a function of mass. Objects more massive than $4 \times 10^{13} M_{\odot}$ are detected at more than $5 \sigma$ at all redshifts, a limit extending well down into the relatively unexplored group range (red line). PRISM is able to measure peculiar velocities over most of the cluster range, i.e., for $M>2 \times 10^{14} M_{\odot}$ (dashed blue line), and relativistic effects, giving access to cluster temperature, for the more massive systems (dot-dashed green line), both out to high redshift. Note that in practice, the detection depth will be lower close to the galactic plane, where a fraction of the clusters will inevitably be missed (although this should be only a very small fraction of the total).

sensitivity of order $1 \mu \mathrm{K}$ or better per resolution element). Similarly maps of the intensity and polarization of all main emission processes will be made available. These include (1) emission of all galactic components in absolute intensity and polarization (including main spectral lines such as lines of CO, C-I, C-II, N-II, O-II, HCN, HCO+), (2) maps of thermal (tSZ) and kinetic (kSZ) Sunyaev-Zel'dovich effect, (3) maps of CIB emission in various redshift shells, (4) maps of the lensing potential and of the matter density in different redshift shells, and (5) several catalogues of various galactic and extragalactic objects including a catalogue of about a million galaxy clusters and large groups up to redshift $z>3$, and (6) catalogues of thousands of very high redshift dusty galaxies, strongly lensed galaxies, and high redshift proto-clusters.

\section{Probing the Universe with galaxy clusters}

Galaxy clusters are an invaluable and proven cosmological tool. The SZ effect is playing an increasingly major role in the exploration of our Universe at large redshift [e.g., 3, 6, 14, 53, 86, 162, 182]. Using the thermal Sunyaev-Zeldovich effect, PRISM will produce the largest galaxy cluster catalog ever, with far more objects than any past or planned cluster survey and, more importantly, extending to higher redshifts. Cluster and group systems will be detected by PRISM throughout our Hubble volume from the moment they first emerge. With the kinetic SZ effect, PRISM will map the cosmic peculiar velocity field throughout the entire observable universe, an objective that cannot be attained by any other means and which will provide a new cosmological probe. PRISM will furthermore provide cluster mass determinations out to high redshift through gravitational lensing of the CMB in both temperature [192] and polarization [124]. PRISM's high angular resolution and frequency coverage in bands unreachable from the ground bring these objectives within reach. The Planck, ACT, and SPT experiments demonstrated the potential of the SZ effect for studying 
galaxy clusters and using them to constrain cosmological models. PRISM will transform SZ cluster studies into arguably our most powerful probe of cosmic large-scale structure and its evolution.

\subsection{Characterizing the PRISM cluster catalog}

We forecast the content of the PRISM SZ catalog by applying a multi-frequency matched filter [MMF, 138] to a simulated typical field at intermediate Galactic latitude. Our detection mass remains below $5 \times 10^{13} M_{\odot}$ at all redshifts (figure 1), and extrapolating from the observed Planck counts, we predict more than $10^{6}$ clusters, with many thousands at $z>2$. The primary source of uncertainty in this prediction lies in the adopted SZ signal-mass scaling relation, $Y-M$. Fortunately, this is more clear than prior to Planck. We now know from Planck SZ observations $[168,170]$ that the SZ signal scales with mass according to our adopted relation down to much smaller masses in the local universe, leaving as our main uncertainty the presently poor knowledge of its redshift dependence. PRISM will determine this evolution, through its mass measurement capabilities (see below), and constrain cosmology from the observed cluster evolution.

One of the notable strong points of PRISM is its ability to measure cluster masses at all redshifts through gravitational lensing of the CMB anisotropies. Using detailed lensing simulations, we estimate that a cluster mass of $2 \times 10^{14} M_{\odot}$ will be detected by PRISM at all redshifts with a signal-to-noise of unity (Melin \& Bartlett 2013, in preparation). We will therefore be able to measure individual masses for the more massive systems. More importantly however, we can measure the mean mass of clusters through binning as a function of SZ signal (or other observable) and redshift. This gives us the essential capability to determine the scaling relations with cluster mass necessary for cosmological interpretation of the cluster counts and for structure formations studies. It is important to note that PRISM is self-sufficient in this critical aspect of cluster science.

PRISM will surpass all current and planned cluster surveys, including eROSITA and Euclid - not just in total number, but most importantly in number of objects at $z>$ 1.5. Because optical/NIR cluster searches suffer from high contamination rates, especially at redshifts beyond unity, cluster identification and the construction of a precise selection function will be vastly more robust for PRISM than for Euclid. Only PRISM can find a significant number of clusters in the range $2<z<3$, the critical epoch that current observations identify as the emergence of the characteristic cluster galaxy population on the red sequence. PRISM will also enable us to explore the abundance of the intra-cluster medium (ICM) through the $Y-M$ relation and its relation to the galaxy population at these key redshifts.

At the time of operation, large imaging (e.g., DES, LSST, HSC) and spectroscopic surveys (e.g., 4MOST, PFS, WEAVE, BigBOSS/MS-DESI, SKA) will have covered the entire extragalactic sky. We can therefore easily obtain redshifts, spectroscopic or photometric, for all objects up to $z=2$, and the two micron cutoff of Euclid's IR photometric survey ( $\mathrm{H}$ band) will be sufficient to detect the $4000 \AA$ Abreak in brighter cluster galaxies at higher redshifts. Follow-up with ALMA or CCAT can provide additional information (e.g., redshift, structure, object population) of the most interesting objects.

\subsection{Thermal SZ maps}

The impressive performance of PRISM for cluster detection throughout our Hubble volume is illustrated by a realistic simulation of PRISM observations on a small patch of sky of 

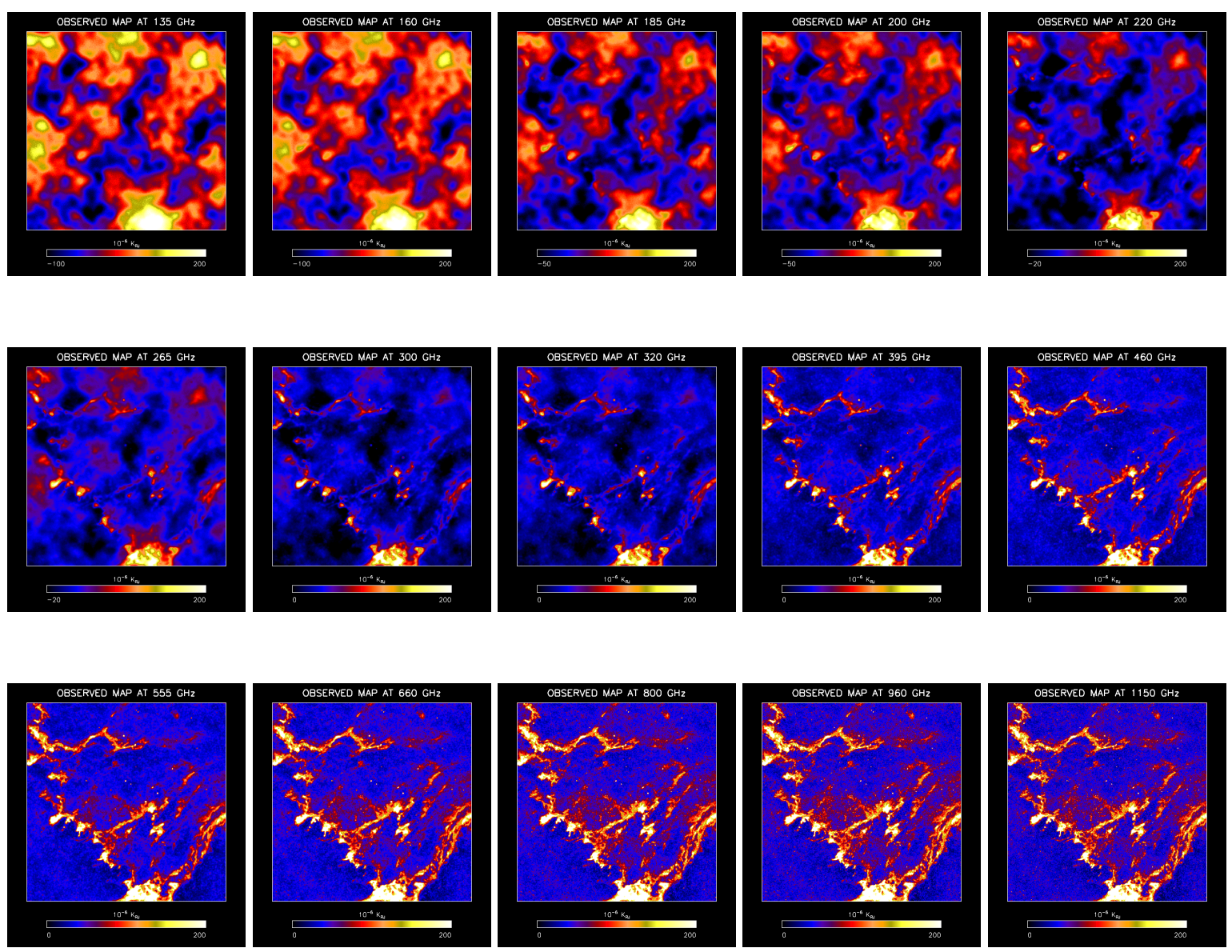

Figure 2. Simulated PRISM observations in 15 broadband channels from 135 to $1150 \mathrm{GHz}$. The top left map, at $135 \mathrm{GHz}$, is dominated by CMB temperature anisotropies, and is observed with 3.8' angular resolution. At $1150 \mathrm{GHz}$ (bottom right), the angular resolution is 27" and the emission is dominated by Galactic dust, nearby galaxies, and CIB emission. The thermal SZ emission is subdominant at all frequencies.

about 10 square degrees $(3.4 \times 3.4$ degrees $)$ through a region of low dust emission in the Draco constellation. Figure 2 shows simulated observations in 15 frequency channels ranging from 135 to $1150 \mathrm{GHz}$ in intensity only. The simulation comprises thermal dust and infrared sources scaled from Herschel observations using the most recent dust model being developed within the Planck collaboration, a model for the cosmic infrared background generated by a population of high redshift proto-spheroidal galaxies [27, 215], as implemented in the Planck Sky Model [60], thermal SZ emission from a population of clusters and large groups matching Planck number counts and scaling relations [162], kinematic SZ signal generated following the approach of Delabrouille, Melin and Bartlett [62], and CMB anisotropies matching the recent Planck power spectrum and cosmological constraints. Other Galactic foreground emission (synchrotron, free-free, spinning dust, and molecular lines) and radio sources will not contaminate SZ cluster detection significantly after being subtracted out using PRISM's lower frequency data and narrow band observations. They are therefore neglected for this simulation.

The SZ thermal emission signal (tSZ) is recovered from the maps using the so-called Internal Linear Combination (ILC) method over a set of spectral windows, which define a set 

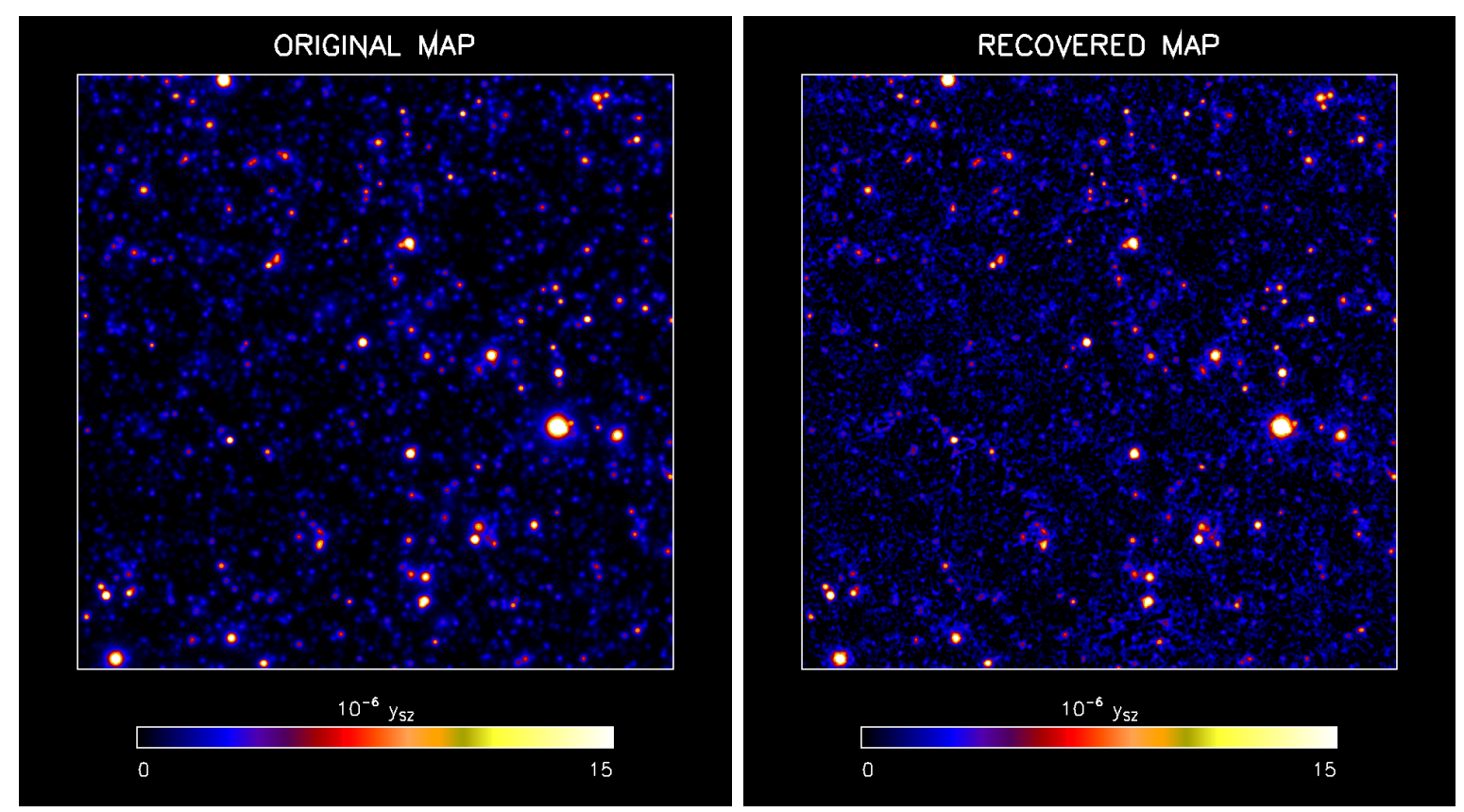

Figure 3. Extraction of thermal SZ emission from a set of simulated PRISM maps. Most of the clusters in the original map are clearly visible after separation of the tSZ component with a straightforward multiscale ILC. Hundreds of SZ clusters are detectable on this very small patch $(0.025 \%$ of sky).

of scales adjusted to match the resolution of the different observed maps. A thermal SZ map is first obtained at 3.8' resolution using all 15 bands, each smoothed to 3.8'. Fourteen maps at 3.2' resolution (corresponding to that of the $160 \mathrm{GHz}$ channel) are then computed from all maps at frequencies above $160 \mathrm{GHz}$. We subtract from these 14 maps the corresponding $3.8^{\prime}$ resolution maps, to obtain 14 maps with structure on scales ranging from 3.2 to 3.8 arcminutes. An ILC on these 14 maps is performed to produce a map of thermal SZ emission on scales 3.2-3.8 arc-minutes, which is added to the lower resolution tSZ map. The process is iterated using each time only observations in the highest frequency channels until a tSZ map at 1.3' resolution, corresponding to the beam of the $395 \mathrm{GHz}$ channel, is obtained. Considering that the process is localized in pixel space by being applied to a small patch of sky, this implements an ILC that is localized in both pixel space and across scales, similar to the needlet LC method successfully used for CMB extraction on WMAP data [8, 9, 61].

Figure 3 illustrates the outcome of this processing. Several hundred clusters matching the input simulation are visible in the recovered tSZ maps, demonstrating that a straightforward component separation procedure makes it possible to observe most of the original clusters. The number of clusters extracted from the recovered tSZ map in this simulation confirms the predicted cluster number counts obtained with the MMF method, supporting the estimated number of $10^{6}$ clusters or more observed by PRISM. In addition, the recovered map can also be exploited by stacking regions centered on various targets: very faint candidate optical clusters not individually detected, expected filaments of the cosmic web between pairs of detected clusters, very distant candidate massive proto-clusters detected by the infrared red emission of their dust emission, distant quasars, HI galaxies, etc. 

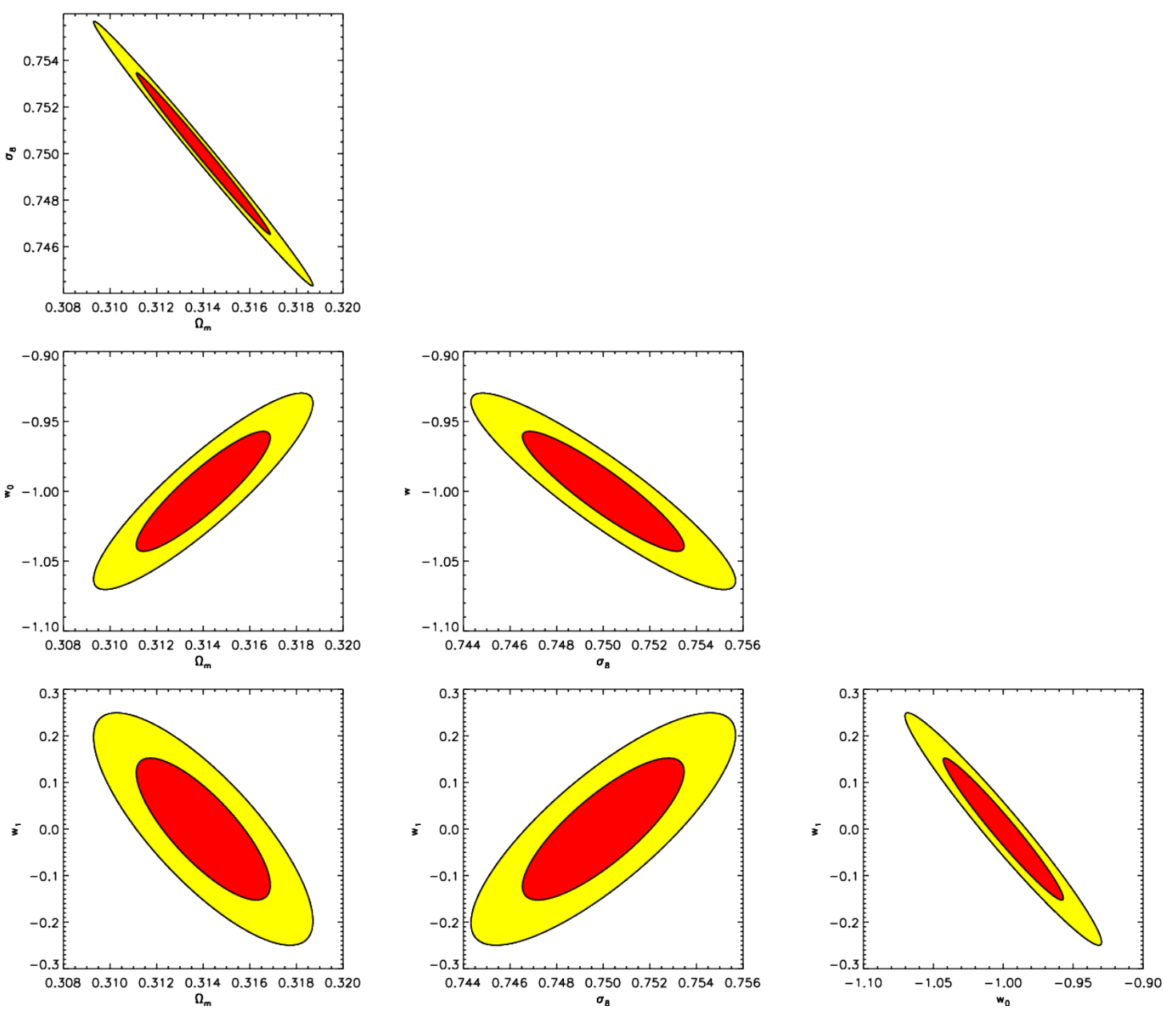

Figure 4. Cluster constraints on a four parameter fit with $\Omega_{\mathrm{m}}, \sigma_{8}$, and dark energy equation-ofstate parameters $w_{0}$ and $w_{1}$. Note in the top left panel the tight constraints in the $\Omega_{\mathrm{m}}-\sigma_{8}$ plane, marginalized over $w_{0}$ and $w_{1}$, and in the bottom right panel the constraints in the $w_{0}-w_{1}$ plane, marginalized over $\Omega_{\mathrm{m}}$ and $\sigma_{8}$, corresponding to a dark energy FoM of 992 .

\subsection{Cluster counts as a cosmological probe}

As an example of the cosmological constraints that can be obtained from the expected cluster catalog, we performed a standard Fisher analysis with the results shown in figure 4 . The bottom right panel, in particular, shows constraints on the dark energy equation-of-state parameters $w_{0}$ and $w_{1}$ for a four-parameter run, marginalized over $\Omega_{\mathrm{m}}$ and $\sigma_{8}$ in a standard flat $\Lambda$ CDM model. The one-dimensional constraints on the dark energy parameters are $w_{0}=-1 \pm 0.003$ and $w_{1}=0 \pm 0.1$. The figure-of-Merit (FoM) quantifying the constraining power on the dark energy equation-of-state parameters is $\left(\operatorname{Det}\left(\operatorname{Cov}\left[w_{0}, w_{1}\right]\right)\right)^{-1 / 2}=992$ from the cluster counts alone.

Structure evolution is sensitive to neutrino mass, which dampens growth at late times due to free-streaming and reduces the amplitude of matter perturbations as measured by $\sigma_{8}$. Cluster counts are therefore a powerful probe of neutrino mass [186], given constraints from the primary CMB anisotropies. For instance, changing the total neutrino mass from 0.057 to $0.096 \mathrm{eV}$ (lower limits for normal and inverted hierarchy respectively) changes the value 
of $\sigma_{8}$ by 0.01 , three to four times the standard deviation of the contour ellipses in figure 4 . Despite their simplicity, these examples illustrate the power of the PRISM cluster catalog as a cosmological probe.

The above forecasts rely only on the cluster abundance data. However, PRISM will also provide extensive data on the large-scale distribution of detected clusters that can be exploited for correlation analysis on large scales, or a large scale structure (LSS) density distribution reconstruction that has an equivalent and complementary constraining power for cosmological parameters, and hence will improve the overall result. Here there may be a very unique application of the data: in some models, Dark Energy can have LSS fluctuations on very large scales (i.e., on GpC scales, where they are not damped) [132]; PRISM is likely to be the first experiment to provide the observations that can probe the LSS with clusters on these very large scales.

\subsection{Cosmic velocity field}

PRISM will map the peculiar velocity field through the kinetic SZ effect [kSZ, 19, 184, 202], providing a new, independent probe of dark matter and large-scale structure evolution. The mass limit to which we expect to measure a velocity of $300 \mathrm{~km} / \mathrm{s}$ to $5 \sigma$ on individual clusters is about $2 \times 14 M_{\odot}$ at $z<2$ (see blue line in figure 1). This mass limit implies that PRISM will obtain velocity measurements for $\sim 2 \times 10^{5}$ clusters out to the highest redshifts, and can further constrain velocity flows on large scales by stacking individual measurements below that detection threshold in cells of solid angle and redshift. Peculiar velocities directly trace the matter distribution. Measurements of the evolution of cosmic velocities therefore complement lensing measurements of the mass distribution; in fact, velocities provide much more local information on the matter distribution than lensing, which measures the mass projected along the line-of-sight over the broad redshift range of the lensing efficiency kernel.

While the relation between velocity and mass distributions is fixed in standard gravitational theory, the relation is often violated in modified gravity theories. By comparing measured velocities to mass concentrations, for example from Euclid lensing, galaxy surveys (including dusty galaxies and CIB fluctuations detected by PRISM - see section 4), and CMB lensing correlated with mass tracers, we can test the theory of gravity on cosmic scales and to high redshift. This science is unattainable by any other means, and PRISM will fully exploit this untapped avenue [e.g., 92].

\subsection{Patchy reionization}

The kSZ effect also generates non-Gaussian anisotropy on the sky, with contributions from the epoch of reionization because of variations in the ionization fraction related to the patchiness of the process [136]. At $\ell \simeq 1000-2000$ the CMB power spectrum has a substantial contribution due to this signal. PRISM has the sensitivity to constrain the duration of reionization and the characteristic bubble size of the ionization regions, informing us about the nature of the first sources [137]. The small change in the small-scale CMB power spectrum caused by the patchy optical depth screen $[68,141]$ should also be detectable.

\subsection{Relativistic and non-thermal effects}

Relativistic corrections to the thermal SZ spectrum depend on cluster temperature and optical depth $[19,32,105,144,145,183,191]$. They are small for low temperature clusters 
$(T<4 \mathrm{keV})$ but become relevant for hot clusters. Wide spectral coverage combined with arcminute angular resolution enable PRISM to map the temperature of clusters in an independent way from X-ray measurements. As shown in figure 1, we will determine the temperature of clusters down to a mass limit just above $10^{14} M_{\odot}$. Since X-ray measurements depend on the square of the electron density and the SZ effect depends linearly on this density, differences in the X-ray and SZ determined temperatures reveal interesting physics. Furthermore, cluster temperature, like the integrated SZ signal, is a good cluster mass proxy. Comparing the two with each other and also with lensing masses will deepen our understanding of cluster scaling relations, critical to cosmological studies with clusters.

Combined with the thermal SZ signal, the temperature measurement gives us a determination of the cluster gas mass. The is needed to calibrate the optical depth as a function of SZ signal, required to extract velocities from the $\mathrm{kSZ}$ signal. Moreover, evolution of cluster gas mass is central to understanding the baryon cycle of cooling and feedback at the heart of galaxy formation With PRISM we can study gas mass in individual cluster systems to the highest redshifts where we detect clusters, thanks to the unique distance independence of SZ signals.

In addition to the thermal relativistic effect, non-thermal processes leave their imprint on the spectrum of the SZ effect. These could signal the presence of highly energetic particles, dark matter annihilation products, AGN outflows, intracluster cavities and shocks [45-47, 49$51]$. We may even study the temperature structure of the most massive systems $[48,59]$ and derive the electron distribution function [176].

\subsection{Diffuse SZ and the cosmic web}

The diffuse, unresolved SZ effect probes a different mass and redshift range than observations of individually detected objects. PRISM will study this diffuse effect through the power spectrum and higher order moments of an SZ map of the sky, such as shown in figure 3. Planck recently extracted the first all-sky Compton parameter ( $y$-fluctuation) map [163], but the results are limited by foregrounds and noise. With many more spectral bands and much better sensitivity and resolution, PRISM will significantly improve the results. With its ability to isolate and subtract detected clusters over a vast range of mass and redshift, PRISM can study the diffuse gas in the remaining, smaller halos, and even within the cosmic web itself.

Apart from this statistical measure, we will also be able to directly map the gas content of the cosmic web in the larger filaments. A significant fraction of the baryons of the Universe are expected to reside in the warm-hot intergalactic medium (WHIM) and remain undetected at low redshifts. The WHIM phase, with over-densities between 5 to 200 times the critical density and temperatures around $10^{5}-10^{7} \mathrm{~K}$, is expected to reside mostly in filaments, but also around and between massive clusters [30,31]. The SZ thermal pressure contribution of this cosmic web of gas [64] is potentially detectable with PRISM, either by direct detection of the bridge of inter-cluster matter between galaxy clusters [e.g., 167], or even the individual filaments around the brightest clusters. This provides important information on the physical state of the baryons in this little understood, moderately dense region of the structure hierarchy.

We will explore the gas content of dark matter halos down to very low masses, a research area pioneered by Planck by stacking SZ measurements based on known objects to detect the signal down below $10^{13}$ solar masses $[168,170]$. This measurement over such a vast mass range is unique to the SZ effect and a highly valuable constraint on feedback mechanisms 
at the heart of galaxy formation. PRISM greatly expands this important science area by pushing to the lowest possible masses and accessing a broad redshift range. We will probe the gas content and its evolution in halos hosting different types of objects and as a function these object properties. Combined with PRISM's lensing measurements, we will wield a fundamentally new and exceptionally powerful tool to study the relation between luminous and dark matter.

\subsection{Polarized SZ effect}

The polarized SZ signal is generated by any quadrupole CMB anisotropy seen at the position of a cluster, such as created by cluster peculiar velocity or the primordial CMB quadrupole itself [180]. Measurements of the polarized SZ signal therefore give access to the transverse component of cluster velocities and a way to estimate the CMB quadrupole at distant locations.

By stacking measurements of the polarized SZ signal from PRISM, we will attempt to estimate the CMB quadrupole at different positions within the Hubble volume. This will allow us to reduce the cosmic variance on this multipole that spans the largest cosmic scales. Averaging the signal of thousands of clusters at high redshift in opposite directions of the sky will dampen the effect from their peculiar velocity component, leaving the component that is proportional to the intrinsic CMB quadrupole. Such an estimate of the quadrupole as seen by objects at high-z would help understand why the low quadrupole seen by Planck and WMAP seems to be at odds (marginally) with the standard model. Kamionkowski \& Loeb (1997), Sazonov \& Sunyaev (1999) and others show that the expected polarization signal is at the level of $10^{-2} \mu \mathrm{K}$ for typical clusters, which could be achieved by an experiment with sub- $\mu \mathrm{K}$ after averaging over thousands of clusters.

X-ray observations from Chandra reveal the presence of cold fronts associated with fast moving (supersonic) bulk motions of the gas in the core region. These bulk motions leave an imprint in the kinetic SZ (proportional to the radial component of the velocity) and also on the polarization pattern of scattered CMB photons (proportional to the square of the tangential component of the velocity). As shown by Diego, Mazzotta, \& Silk (2003), in a typical cluster one expects $\mathrm{kSZ}$ distortions of order $50 \mu \mathrm{K}$ and polarization intensities of 0.3 $\mu \mathrm{K}$ associated with these bulk gas motions. Although $50 \mu \mathrm{K}$ should be easily detected by PRISM, the polarization will prove more challenging; however, in extreme cases like the bullet cluster, where the velocity is significantly higher, the polarization could be boosted to the level of $1 \mu \mathrm{K}$ (due to the squared dependence of the polarization intensity with the velocity) and be detected by PRISM. Combining the kSZ (radial component) with the polarized SZ (tangential) would allow a determination of the three-dimensional direction of the velocity, opening the door to novel dynamical studies of galaxy clusters.

\section{Extragalactic sources and the cosmic infrared background}

\subsection{Early evolution of galaxies}

Although Herschel and Spitzer produced spectacular advances in our understanding of early, dust enshrouded phases of galaxy evolution, our knowledge of star-formation history in the distant universe is still largely incomplete. The PRISM mission will yield essential progress thanks to its unique properties: full sky coverage and unparalleled frequency range.

As illustrated in figure 5, PRISM's unprecedented frequency coverage provides direct measurements of the bolometric luminosities of star-forming galaxies from $z$ of a few tenths 
up to $z \approx 6-7$. At $z \gtrsim 2$, i.e. in the redshift range where both the cosmic star formation and the accretion rate onto supermassive black-holes are maximum, both the IR peak associated to the dusty torus around the AGN $\left(\lambda_{\mathrm{p}, \mathrm{AGN}} \approx 30 \times(1+z) \mu \mathrm{m}\right)$ and the peak of dust emission in the host galaxy are within the covered range.

Moreover, measurements of the complete far-IR to mm-wave SEDs will vastly improve the accuracy of photometric redshift estimates that have a rms error of $\approx 0.2(1+z)$ with SPIRE alone [118]. This means that the PRISM survey will allow us to characterize with high statistical accuracy the evolution with redshift of the bolometric luminosity function, bypassing the need of applying uncertain bolometric corrections.

At $z \gtrsim 2$ analyses of the full SEDs will return information on the evolution of the relationships between star-formation and nuclear activity: what fraction of the bolometric energy radiated by star-forming galaxies is produced by accretion onto supermassive black holes in active galactic nuclei (AGN)? What are the evolution properties of far-IR selected AGNs? What fraction of them is associated to active star formation? Are the growth of central super-massive black hole and the build-up of stellar populations coeval, or does the AGN evolution occur earlier as indicated by some recent data [12]? The substantially higher spatial resolution (thanks to the shorter wavelength channels) and the correspondingly higher positional accuracy compared to Herschel/SPIRE ${ }^{1}$ will greatly improve the identification of reliable counterparts in other wavebands (X-ray, UV, optical, near-infrared, radio), necessary for a comprehensive understanding of the properties of detected galaxies.

Millimeter-wave observations of low- $z$ dusty galaxies will measure their free-free emission, that remained elusive so far. For example only for three galaxies detected by Planck this emission was clearly identified [151]. PRISM will increase the sample to a few hundred galaxies. The free-free emission is a direct measure of the star-formation rate (SFR), unaffected by extinction and not contaminated by the effect of old stellar populations that can contribute to dust heating and, therefore, to the far-IR emission. A comparison of the estimates of the SFR coming from the free-free emission with those from the far-IR will disentangle the contributions to the latter from young stars and from the evolved stellar population, thus providing insight into the intensity of the interstellar radiation field and on the heating of the interstellar dust.

Its all-sky coverage makes PRISM uniquely suited to study rare phenomena. Examples are the 'maximum starburst' galaxy at $z=6.34$ detected by Herschel/SPIRE [185] or the hyper-luminous IR galaxies (HyLIRGs) such as the binary system, pinpointing a cluster of star-bursting proto-ellipticals at $z=2.41$, discovered by [106]. The estimated surface density of the former objects is of $\sim 0.01 \mathrm{deg}^{-2}$, that of binary HyLIRGs of $3-9 \times 10^{-2} \mathrm{deg}^{-2}$, implying that PRISM will detect from several hundreds to thousands of such objects. The $z=6.34$ galaxy was found when looking for ultra-red sources with flux densities $S_{250 \mu \mathrm{m}}<S_{350 \mu \mathrm{m}}<$ $S_{500 \mu \mathrm{m}}$. The PRISM survey will allow us to look for even redder sources, potentially at even higher redshifts, and will provide a test of our understanding of the interstellar medium and of the star-formation under extreme conditions.

Strongly gravitationally lensed systems have long been very difficult to identify in sufficiently large numbers to be statistically useful. This situation changed drastically with the advent of (sub-)mm surveys. One of the most exciting Herschel/SPIRE results was the direct observational confirmation that almost all the galaxies brighter than $\approx 100 \mathrm{mJy}$ at $500 \mu \mathrm{m}$ are either strongly lensed or easily identifiable low- $z$ spirals plus a small fraction of flat-

\footnotetext{
${ }^{1}$ Note that deep Herschel/PACS surveys have covered only a few $\operatorname{deg}^{2}$. The large area Herschel surveys have taken PACS data in parallel mode and have shallow detection limits.
} 

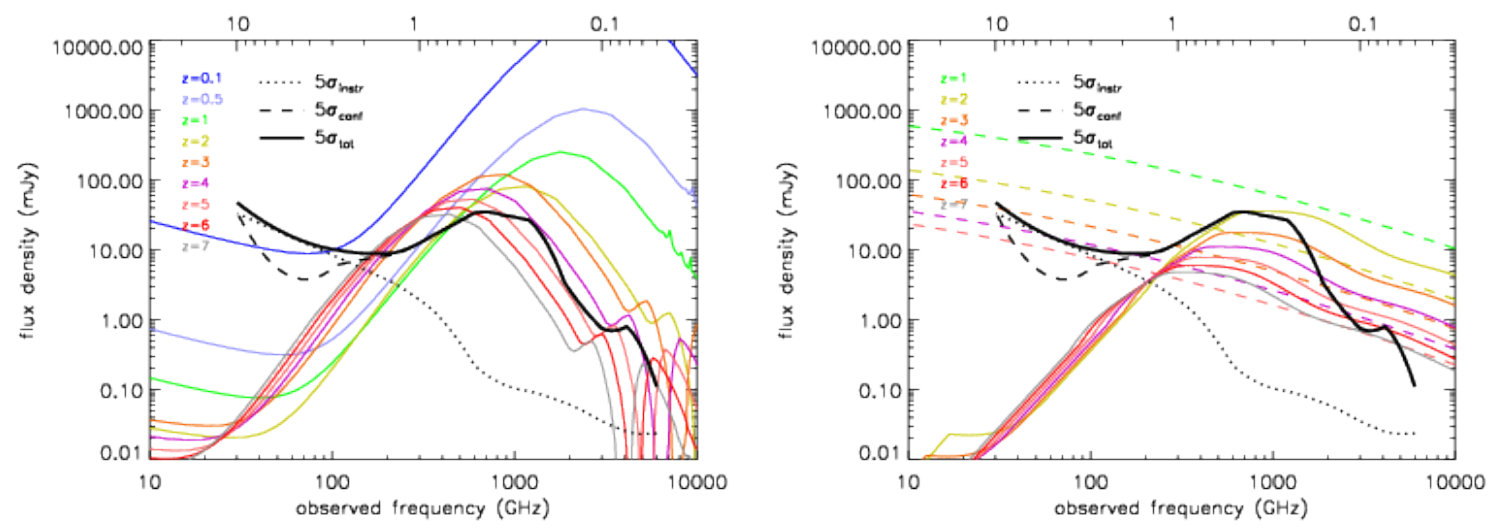

Figure 5. SEDs of dusty galaxies (left panel) and of AGNs (right panel) at different redshifts compared with estimated $5 \sigma$ detection limits (solid black line) taking into account instrumental and confusion noise summed in quadrature. The instrumental noise refers to the full mission, in 1 arcmin. pixels. The $5 \sigma$ detection limits allowing for either component are shown by the dotted and the dashed black lines, showing that PRISM is confusion limited above $\approx 150 \mathrm{GHz}$. We have assumed that component separation techniques, extensively validated both on simulations and on real data, can efficiently remove diffuse emissions such as the CMB (that would otherwise dominate the fluctuation field for $\nu \lesssim 220 \mathrm{GHz}$ ) and Galactic emissions. In the left panel, at $z=0.1$ and 0.5 we have plotted the Arp 220 SED scaled to an IR $(8-1000 \mu \mathrm{m})$ luminosity of $10^{12} L_{\odot}$. At $z \geq 1$ we have used the SED of the $z \approx 2.3$ galaxy SMM J2135-0102 scaled to $L_{\mathrm{IR}}=10^{13} L_{\odot}$ for $z=1$ and $z=2$, and to $L_{\mathrm{IR}}=3 \cdot 10^{13} L_{\odot}$ [the luminosity of the $z=6.34$ galaxy detected by Herschel/SPIRE, 185] for $z \geq 3$. In the right panel, the solid colored lines represent SEDs of a type-2 QSO (contribution of the host-galaxy subtracted) with $L_{\mathrm{IR}}=10^{13} L_{\odot}$ at several redshifts $\geq 2$, while the dashed colored lines show a schematic representation of the SED of the prototype blazar 3C 273 shifted to redshifts from 1 to 5 .

spectrum radio quasars [142]. The surface density of high- $z$ strongly lensed galaxies above this limit is $\approx 0.3 \mathrm{deg}^{-2}$, implying that an all-sky survey can detect $\sim 10^{4}$ such systems. The typical magnification is of a factor $\mu \sim 10$. The fact that these sources are very bright makes redshift measurements with $\mathrm{CO}$ spectrometers and high resolution imaging with millimeter interferometers (ALMA, NOEMA, SMA,...) relatively easy. This will allow us to get detailed information on obscured star formation in the early Universe and on processes driving it, in observing times a factor $\approx \mu^{2}$ shorter that would be possible without the help of gravitational amplification and with effective source-plane resolution $\approx \sqrt{\mu}$ higher than can otherwise be achieved.

Optical spectroscopy of galaxies acting as lenses can be exploited to measure the mass distribution of their dark matter halos as a function of redshift. Note that Euclid will directly provide redshifts for the majority of the lenses out to $z \sim 1$ in its area. Taking into account the large number of newly identified strongly lensed galaxies, this will allow a direct test on the evolution of large-scale structure by measuring the mass distribution of dark matter halos as a function of redshift.

Samples of thousands strongly lensed galaxies are also essential for many other astrophysical and cosmological applications [207]. Further constraints on the evolution of large scale structure, hence on cosmological parameters that drive it, can be obtained from the cross-correlation between background sub-mm galaxies and foreground optical or IR galaxies induced by weak lensing $[93,214]$. 
Large numbers of strongly lensed galaxies are also expected from large area optical surveys. It should be noted, however, that sub-mm selection has important distinctive properties. The selected lensed galaxies are very faint in the optical, while most foreground lenses are passive ellipticals, essentially invisible at sub-mm wavelengths so that there is no, or little, contamination between images of the source and of the lens. This makes possible the imaging of lensing events with small impact parameters. Also, compared to the optical selection, the (sub-)mm selection allows us to probe earlier phases of galaxy evolution.

PRISM will study the angular correlation function of detected sources with much better statistics than was possible with Herschel's extragalactic surveys that, altogether, cover little more than $2 \%$ of the sky. Also, the accurate photometric redshifts will allow us to follow the evolution of clustering with cosmic time. Clustering properties measure the mass of dark matter halos associated to galaxies and are a powerful discriminant for galaxy formation and evolution models [215]. In particular this study will allow us to detect high- $z$ proto-clusters of dusty galaxies. We thus investigate an earlier evolutionary phase of the most massive virialized structures in the Universe. This science is possible only in the wavebands covered by PRISM.

The PRISM clustering data will extend to much higher redshift than Euclid, whose wide-area survey will accurately map the galaxy distribution up to $z \sim 1$. The PRISM data will provide information primarily over the redshift range $2<z<3$, corresponding to the peak in star formation activity. Moreover, optical and near-IR data severely underestimate the SFR of dust obscured starbursts and may entirely miss these objects, which are the main targets of far-IR/sub-mm surveys such as PRISM. Only the combination of PRISM and Euclid data will provide a complete view of the spatial distribution of galaxies and of how star formation is distributed among dark matter halos.

\subsection{The cosmic infrared background}

The PRISM sensitivity and spectral coverage will provide substantially improved measurements of the cosmic infrared background (CIB) spectrum with an accurate removal of all contaminating signals. The CIB mean intensity around its peak at $\lambda_{p} \sim 160 \mu \mathrm{m}$ is still quite uncertain [117]. Its determination is obviously crucial to determine the cosmic energy budget and to assess to what extent it has been resolved by existing surveys.

PRISM will also measure, in a uniform way, the CIB power spectrum over an unprecedented range of frequencies and of angular scales (from $\sim 10 \operatorname{arcsec}$ to tens of degrees). Herschel and Planck surveys have provided measurements at $\lambda \geq 250 \mu \mathrm{m}[169,213]$ which, while generally consistent, show significant differences. Estimates of the power spectrum at 100 and $160 \mu \mathrm{m}$, from Spitzer/MIPS data [153] are far more uncertain. The much better statistics due to the much larger area covered by PRISM compared to Herschel and to the much better sensitivity and angular resolution compared to Planck will allow us to obtain a highly accurate assessment of the CIB power spectrum and of its frequency dependence.

On large angular scales the CIB power spectrum measures the linear clustering bias which is a strong function of halo mass. On small scales the clustering properties are determined by the non-linear evolution and probe the processes that drive the formation of multiple galaxies within large dark matter halos. Specifically, the fluctuation level at multipoles $\ell \sim 1000$ at far-IR/sub-mm wavelengths mostly probes the $z<1$ Universe and is very sensitive to the 1-halo term, thus to the effect of environment on star formation [15].

The composition and the redshift distribution of faint sources (below the detection limit) contributing to the CIB varies with frequency. The characteristic redshift of sources 
of the CIB systematically increases with increasing wavelength, from far-IR to mm-waves. This change is related to the origin of the early/late-type galaxy dichotomy, since present-day spheroidal galaxies have been forming most of their stars at $z \gtrsim 1.5$ while stellar populations of late type galaxies are substantially younger and indeed these galaxies have been continuously forming stars up to the present time. The observed downsizing in galaxy evolution implies that the change with frequency of the dominant galaxy population translates in a variation of the clustering amplitude. Thus the frequency dependence of the CIB power spectrum also contains information on the origin of the early/late-type galaxy dichotomy and on its relationship with halo mass.

\subsection{Radio sources}

PRISM will extend the counts of radio sources, both in total and in polarized intensity, by at least one order of magnitude downwards in flux density compared to Planck. Above $217 \mathrm{GHz}$, the counts will be determined for the first time over a substantial flux density range with good statistics. ${ }^{2}$ This will make possible the first investigation of the evolutionary properties of radio sources at (sub-)mm wavelengths.

PRISM will provide measurements of the spectral energy distribution (SED) of many thousands of radio sources and of multifrequency polarization properties for hundreds of them. The vast majority of these sources are expected to be blazars, and the accurate determination of their spectra will allow us to understand how physical processes occurring along relativistic jets shape the SED [156]. We will also get numerous samples of 'extreme' radio sources [157] allowing us to investigate the rich phenomenology of radio sources at (sub-)mm wavelengths.

A few hundred steep-spectrum radio sources will also be detected. The multi-frequency measurements will provide the distribution of break frequencies due to electron aging, allowing an unbiased estimate of the distribution of radio source ages.

These observations will also shed light on the relationship between nuclear radio emission and star formation activity in the host galaxies. In particular, the present notion is that the blazar hosts are giant passive ellipticals, but already Planck has provided examples of catalogued blazars that are hosted by galaxies with strong star-formation activity. This mission will allow us to quantify the abundance of hosts of this kind and to investigate relationships between blazar and host properties.

\subsection{Extragalactic sources and the integrated Sachs-Wolfe effect}

The cross-correlation of radiosource data (NVSS) and CMB maps has bean already probed as a fruitful way to detect the integrated Sachs-Wolfe (ISW) signal. Since the redshift distribution of sub-mm selected galaxies peaks at $z=2-3$, it will be possible, combining the extragalactic IR source catalogue from the survey data with PRISM CMB maps, to measure the Integrated Sachs-Wolfe (ISW) effect out to substantially higher redshift than can be done with Planck + large area optical surveys (limited to $z \lesssim 1$ ). At these high redshifts the ISW is insensitive to a cosmological constant and will probe alternative explanations of the cosmic acceleration. Since the ISW effect shows up on large angular scales, the all-sky coverage of PRISM will allow substantially better statistics than any optical survey. This will be complemented by a similar correlation of PRISM CMB maps with a large catalogue of HI galaxies

\footnotetext{
${ }^{2}$ Sources whose sub-mm emission is synchrotron dominated are rare. The sky coverage of Herschel surveys was insufficient to provide samples large enough to determine their counts.
} 
detected with SKA. In about 1-yr, a SKA survey will observe more than $10^{9}\left(f_{\text {sky }} / 0.5\right)$ HI galaxies in a redshift range $0<z<1.5$, offering yet another view on the impact of dark energy on the dynamics of the large scale structures in our Universe.

\section{Inflation and CMB primordial B-modes}

At the heart of our current Standard Model of the Universe is a set of initial conditions laid down at very early times by what is known as cosmic inflation. During inflation, the Universe undergoes a period of ultra-rapid accelerated expansion. The simplest model involves a slowly evolving fundamental scalar $\phi$, much like the Higgs field, with a potential energy $V(\phi)$ that dominates over its kinetic energy. The energy density of such a scalar field is well approximated by $\rho=V$ while its pressure is $p=-V$. As such, it drives the scale factor of the Universe to evolve approximately as $a(t) \propto \exp (\sqrt{8 \pi G V / 3} t)$.

During this period of inflation, quantum fluctuations of spacetime and the scalar field $\phi$ are amplified and stretched to macroscopic scales. The result is a quasi-Gaussian stochastic distribution of density perturbations with an amplitude $A_{S}$, which depends on scale, or wavenumber $k$. It is convenient to write the dependence of the scalar spectral index as $n_{S}=$ $1+d \ln A_{S}^{2}(k) / d \ln k$. In fact, the theory predicts that $A_{S}$ and $n_{S}$ will depend on the details of $V$ and how itself depends on $\phi$. Furthermore, interactions of $\phi$ with itself and with other fields induce cross-correlations between perturbation modes, leading to non-Gaussianity which can be detected in higher order statistics (bispectrum, trispectrum). Inflation will also generate a bath of primordial gravitational waves (tensor perturbations of the spacetime), which can also be characterized by an amplitude $A_{T}$ and the tensor spectral index $n_{T}=d \ln A_{T}^{2}(k) / d \ln k$. Remarkably, in the simplest models of inflation, the ratio between the tensor and scalar perturbations $r$ is a direct probe of $V$ in the early universe: $r \equiv(T / S)=16\left(A_{T} / A_{S}\right)^{2} \approx$ $M_{P l}^{2}\left(V^{\prime} / V\right)^{2}$. From present observations we have that $V^{1 / 4}=3.3 \times 10^{16} r^{1 / 4} \mathrm{GeV}$, so that measuring $r$ effectively translates into a measurement of the energy scale of inflation.

Clearly, a measurement of $r, n_{S}$, and possibly even $n_{T}$, can directly probe the physics of the early universe for which there is a very rich phenomenology. Single field inflation models can relate $r$ directly with the evolution of $\phi$ at early times. Indeed, for an inflationary expansion lasting long enough to provide the observed level of homogeneity and isotropy, we have that $\Delta \phi / M_{\mathrm{Pl}} \approx(r / 0.01)^{1 / 2}$. Multiple field inflation models can arise in string theory and other proposals for unification at high energies and can lead to even higher values of $r$. In addition, particle and string production during the inflationary period can be an additional source of copious gravitational waves which in some cases will dominate over the usual mechanism. Moreover, very recent models propose that the Electroweak Higgs could also be the inflaton if we allow for nonminimal couplings to gravity. In this case, there is a direct connection between early universe phenomena like inflation with late universe dynamics like dark energy, and future surveys like Euclid could help us discriminate among alternatives.

Over the past few years, the techniques of Effective Field Theory have been applied to the inflationary era allowing a systematic analysis of a broad swathe of theory space. With these techniques it is possible to use constraints on $A_{S}, A_{T}, n_{S}, n_{T}$ as well as non-Gaussianity parameters such as $f_{N L}$ and $g_{N L}$ to place stringent constraints on the types of couplings that are allowed at ultra-high energies. By sifting through these couplings it is then possible to make profound statements about the type of unified theory which should be valid at Planck scales (such as for example Supergravity) and what ingredients should be considered in a 


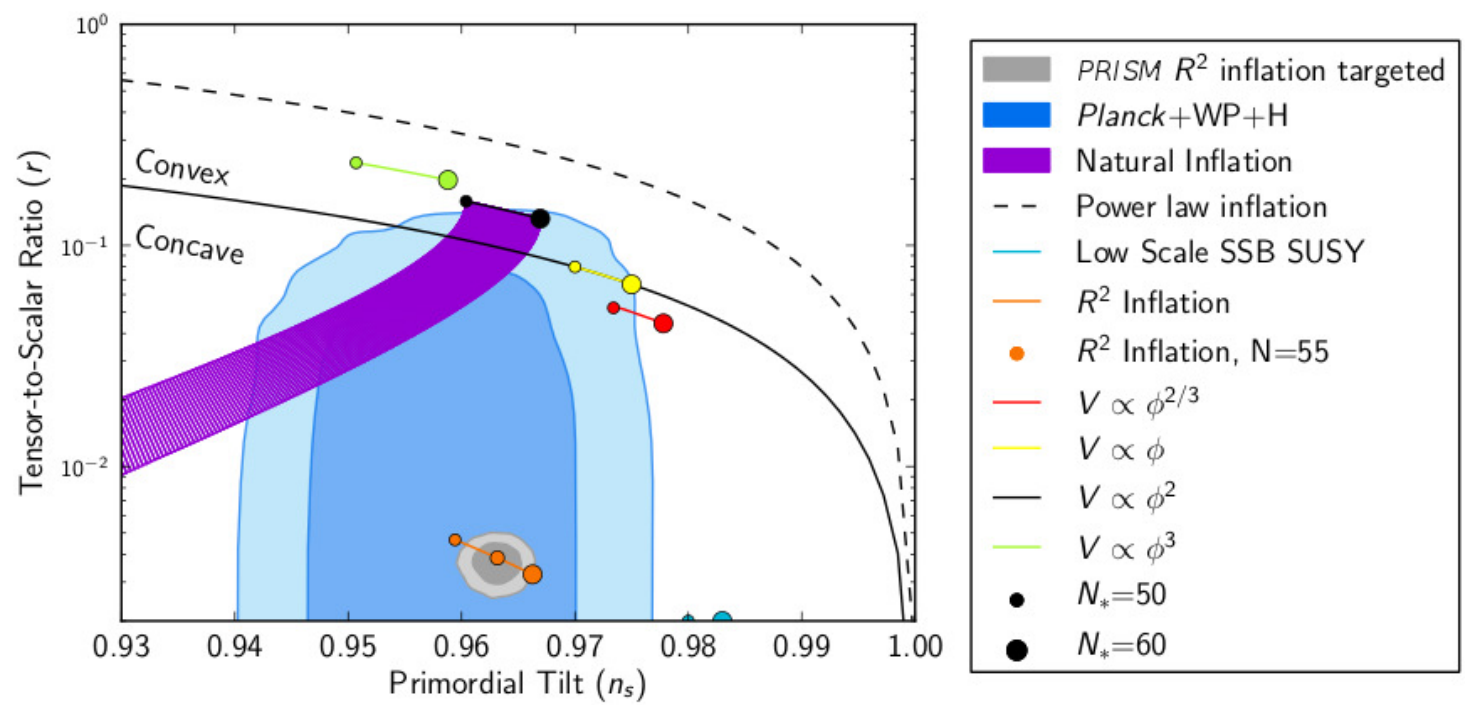

Figure 6. Constraints on inflationary potentials from Planck and the predicted constraints from PRISM (not assuming de-lensing) for a fiducial value of $r=5 \times 10^{-2}$ (adapted from [164]).

quest for ultra-violet completion of our current fundamental theory of particles and fields. Hence, beyond just constraining individual theories, measurements of these quantities can play a crucial role in helping us in the age old quest for a theory of unification.

Primordial gravitational waves imprint a unique, as yet undetected, signature in the CMB polarization. CMB polarization is a spin-two field on the sky, and is decomposed into the equivalent of a gradient — the E-mode - and a curl — the B-mode. Gravitational wave fluctuations are visible as the B-mode polarization of the CMB and are the only primordial contribution to $\mathrm{B}$ relevant at the time of recombination. Hence a detection of B-modes is a direct probe of $r$, and thus the energy scale of inflation and other primordial energetic processes. Furthermore, in the simple case of slow-roll inflation we have that $r \approx-8 n_{T}$. Additional detailed measurements of the shape of the temperature and polarization spectra will measure higher derivatives of the inflationary potential.

The 2013 Planck data release has significantly improved previous constraints on inflationary models. In the simplest $\Lambda \mathrm{CDM}$ scenario, where no tensor perturbations are included, Planck results provide $n_{s}=0.9603 \pm 0.0073$. When tensor modes are included, the same analysis yields $n_{s}=0.9624 \pm 0.0075$ and $r<0.12$ using a pivot scale of $0.002 \mathrm{Mpc}^{-1}$. These results are notable because exact scale invariance (i.e., $n_{S}=1$ ) of primordial perturbations is ruled out at more than $5 \sigma$, a result which still holds when allowing for a variation in primordial Helium abundance and effective neutrino number.

When specific inflationary models are considered, Planck imposes significant constraints on the potential (figure 6), as discussed in ref. [164]. Convex potentials are disfavored by Planck data. Chaotic inflation with $V \propto \phi^{2}$ is excluded at 95\% C.L. for $N_{*}=50$ and only marginally compatible for $N_{*}=60, N_{*}$ being the number of inflation e-foldings. In general, power-law potentials $V \propto \phi^{n}$ for $n>2$ are ruled out. The spontaneously broken SUSY model is disfavored, unless some assumptions on reheating are relaxed. Planck does not have, in general, the power to discriminate among different concave potential shapes. Inflationary models based on extended theories of gravity are also constrained. Remarkably, 
the original $R^{2}$ inflation model proposed by Starobinsky predicts $n_{s}=0.963$ and $r \approx 4 \times 10^{-3}$ for $N_{*}=55$, and is thus fully compatible with Planck observations. Non-minimally coupled Higgs inflation has predictions similar to the $R^{2}$ scenario, and is also well accommodated by Planck.

The Planck results have also led to stringent constrains, via the Effective Field Theory methods, on the broad type of theories which can be at play in the very early universe. In particular couplings between the inflaton and any hidden sector are supressed by of order $10^{-2}$ relative to the energy scale of inflation. With trilinear couplings, the supression can be as large as $10^{-5}$, which for most models of inflation is at the Planck energy scale. This places extremely tight constraint models of string compactification in the supergravity regime, a prime model for unification. Furthermore these constraints begin to enter the quantum gravity regime, opening up a completely new phenomenological window on the Universe. A detection of tensor modes would greatly sharpen these constraints.

In summary Planck has shown that it is possible to test many inflation models using the CMB temperature data, yet even a forecast Planck limit $r<0.05$ would leave many interesting models unprobed. Given that the stochastic background of gravity waves is the smoking gun of inflation, it is crucial to map as accurately as possible the CMB polarization and in particular characterize the B-mode angular power spectrum.

To forecast how well we would be able to measure the power spectrum of the B-modes, it is important to recognize that the foreground signal is likely to dominate the cosmological signal at low $\ell$, where the most constraining information on $r$ is situated. If we propagate the uncertainties connected to foreground contamination into the parameter error forecasts [11, $21,212]$, we find that the proposed experimental set-up will enable us to explore most large field (single field) inflation models (i.e., where the field moves for $\geq \mathrm{M}_{P}$ ) and to rule in or out all large-field models, as illustrated in figure 7.

As the work by [196] indicates, the instrumental sensitivity, angular resolution and, as a result, foreground control and subtraction will enable us to achieve a detailed mapping of the lensing signal, and in particular to implement de-lensing techniques for the measurement of $r$, improving by a factor of three our constraint on $r$. This implies that PRISM will detect $r \sim 3 \times 10^{-4}$ at more than $3 \sigma$. This performance is very close, within factors $\mathcal{O}(1)$, to what an ideal experiment (i.e., with no noise and no foregrounds) could achieve, allowing PRISM to directly probe physics at an energy scale a staggering twelve orders of magnitude higher than the center-of-mass energy at the Large Hadron Collider (LHC).

Figure 8 illustrates the expected noise level of the PRISM CMB observations, in comparison with the CMB temperature and polarization power spectra.

\section{$6 \mathrm{CMB}$ at high resolution}

The temperature anisotropies of the CMB have proved to be a remarkably clean probe of the high-redshift universe and have allowed cosmological models to be tested to high precision. However, the accuracy of the recent results from Planck [159], based on the temperature anisotropies, are now close to being limited by errors in modelling extragalactic foregrounds. Fortunately, further progress can be made with the polarization anisotropies on small angular scales since the degree of polarization of the anisotropies is relatively larger there (around $4 \%$ by $\ell=2000$ ) than the foreground emission. This, combined with the improved resolution and sensitivity of PRISM over Planck and the exquisite frequency coverage for removal of foregrounds, will allow us to extend the current temperature analyses to smaller angular 


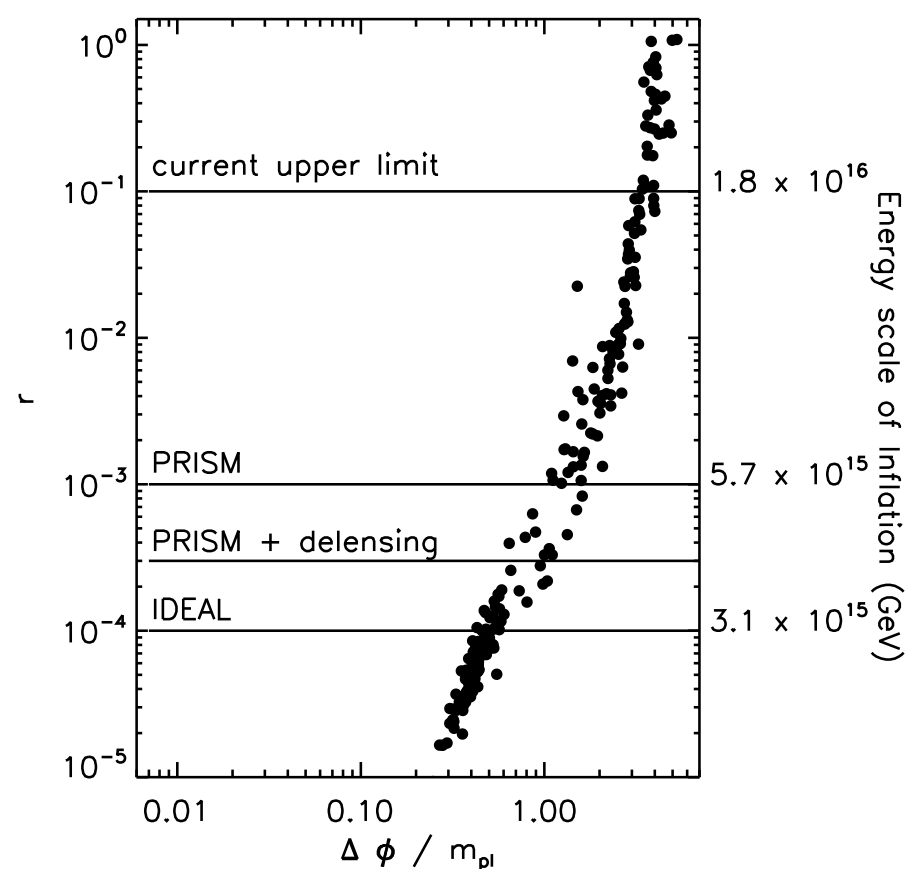

Figure 7. Distribution of inflationary model parameters generated using a model independent approach that Monte-Carlo samples the inflationary flow equations. While these simulations cannot be interpreted in a statistical way (e.g., $[44,115,152])$, they show that models cluster around attractor regions (adapted from [212]).

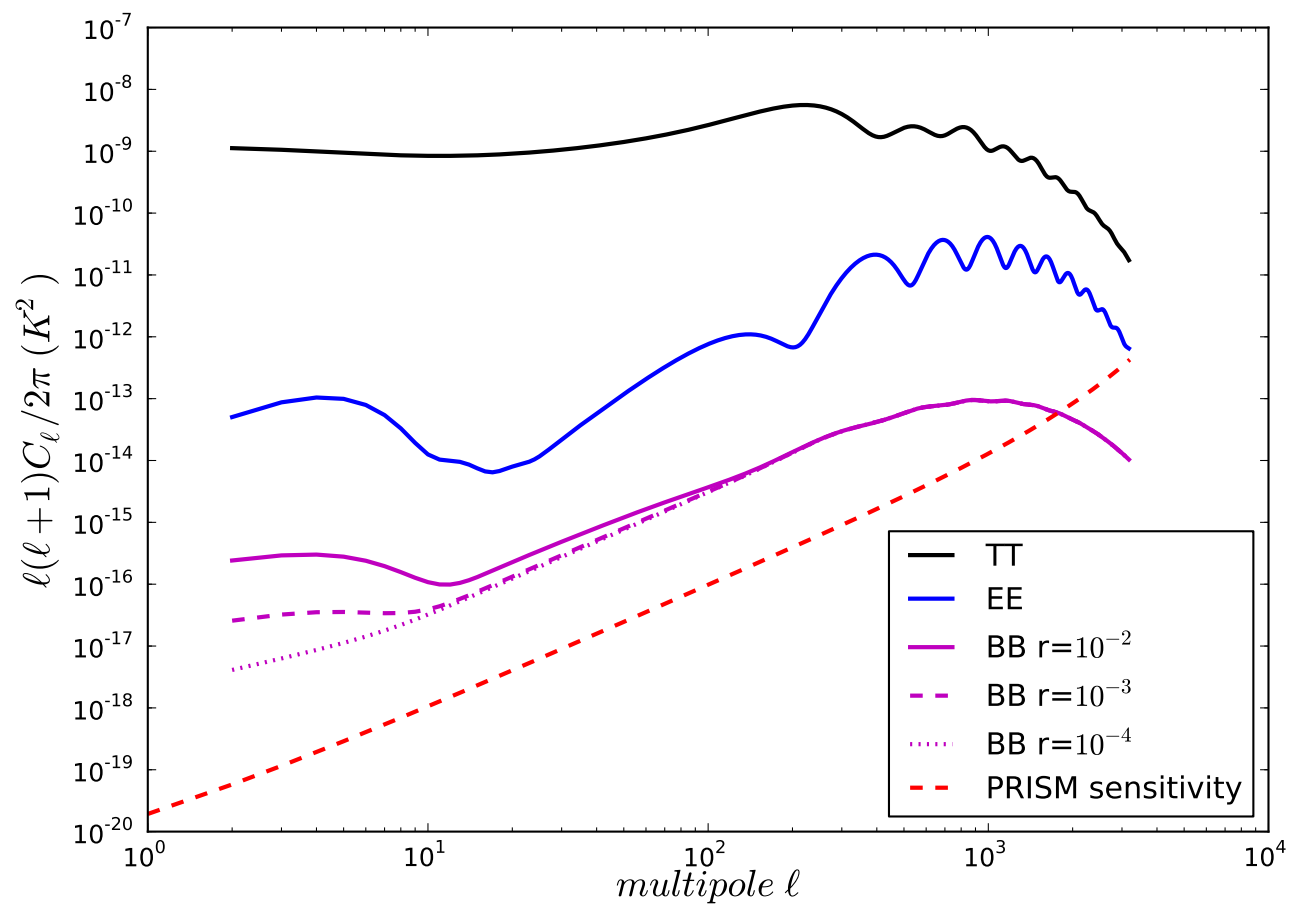

Figure 8. Expected noise level for PRISM observations in individual CMB multipoles. 
scales in polarization. We discuss the main science enabled by such observations - full exploitation of weak gravitational lensing effects in the CMB, improved constraints on earlyuniverse physics from CMB non-Gaussianity, precision tests of extensions to the standard cosmological model from the polarization power spectra in the damping tail, and powerful searches for cosmic strings via the small-scale $B$-mode power spectrum — in the following subsections.

The science case for high-resolution imaging of the CMB is well recognised, and indeed is a major focus of the polarization cameras now operating on the SPT and ACT. While future upgrades to these experiments may cover several $1000 \mathrm{deg}^{2}$ of sky with sensitivity approaching that of PRISM, only a space mission will be able to cover the full sky in many frequency bands. For the science described in this section, the main benefits of a space mission are that it provides uniform calibration over the full sky (important, for example, for localmodel non-Gaussianity searches where one is looking for large-scale modulation of small-scale power), excellent foreground rejection, long-term stability and control of systematic effects, and improved sample variance due to near full-sky coverage.

\subsection{Probing the dark universe with CMB lensing}

Gravitational lensing of the CMB temperature and polarization anisotropies provides a clean probe of the clustering of matter integrated to high redshift (see ref. [125] for a review). The power spectrum of this lensing signal can be used to probe the late-time growth of structure and so access information, such as spatial curvature, dark energy and sub-eV neutrino masses, that are otherwise degenerate in the primary CMB spectra imprinted at recombination [102]. Moreover, by cross-correlating CMB lensing with other tracers of large-scale structure, one can calibrate the astrophysical and instrumental bias relations between the tracers and the underlying density field, which is critical to maximise the returns from future surveys (see e.g. ref. [208]).

Gravitational lensing can be reconstructed from the CMB anisotropies via subtle distortions imprinted on the statistics of the CMB by the lenses [101,217]. This approach has been demonstrated recently for the temperature anisotropies by ACT [57, 58], SPT [211] and Planck [160], with the latter detecting the lensing power spectrum at the $25 \sigma$ level. Lensing reconstruction is statistical, with cosmic variance of the primary anisotropies giving rise to a statistical noise. With only the temperature anisotropies, the lensing $S / N$ cannot exceed unity for multipoles $\ell>300$. However, CMB polarization measurements can greatly improve the $S / N$ since polarization has more small-scale structure than the temperature, and the $B$-modes of polarization are much less limited by cosmic variance of the primary CMB fluctuations [103]. With CMB polarization measurements from PRISM, at a sensitivity of a few $\mu \mathrm{K}$ arcmin and resolution of a few arcmin, it is possible to reconstruct with $S / N>1$ up to multipoles $\ell=600-800$, corresponding to scales of about $15-20 \operatorname{arcmin}$ (see figure 9), over nearly the full sky. Significantly, PRISM can extract all of the information in the deflection power spectrum on scales where linear theory is reliable. Polarization-based lensing reconstructions have recently been demonstrated for the first time by the SPTpol team [85]; cross-correlating an $E-B$ reconstruction with CIB measurements at $500 \mu \mathrm{m}$ from Herschel-SPIRE, they report a $7.7 \sigma$ detection. Significantly, their analysis represents the first detection of the $B$-modes induced by gravitational lensing of the CMB.

The recent ACT, SPT and Planck lensing analyses have further demonstrated the abil-

ity to constrain dark parameters from the CMB alone via lensing. For example, in models with spatial curvature, the combination of the Planck temperature and lensing power spectra 


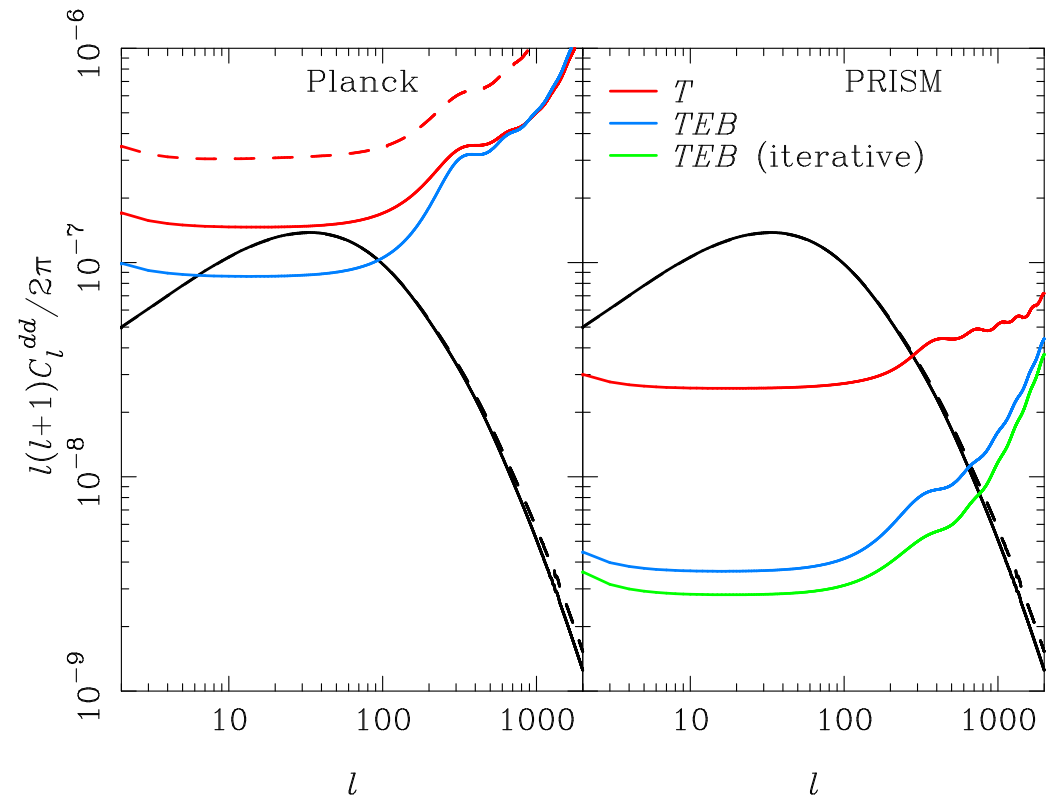

Figure 9. Reconstruction noise on the lensing deflection power spectrum forecast for the full Planck mission (four surveys; left) and PRISM (right) using temperature alone (red) and temperature and polarization (blue). For Planck we also show the approximate noise level for the temperature analysis of the nominal-mission data (red dashed) [160], and for PRISM, we also show the approximate noise level (green) for an improved iterative version of the reconstruction estimator. The deflection power spectrum is plotted based on the linear matter power spectrum (black solid) and with non-linear corrections (black dashed).

measure $\Omega_{\Lambda}$ at $4 \%$ precision, and $\Omega_{K}$ to around $1 \%$ [159]. As an illustration of the constraining power of the lensing measurements from PRISM alone, we consider constraints on the mass of neutrinos. Oscillation data imply that neutrinos must be massive, but the data are insensitive to the absolute mass scale. For a normal hierarchy of masses $\left(m_{1}, m_{2} \ll m_{3}\right)$, the mass summed over all eigenstates is at least $0.06 \mathrm{eV}$, while for an inverted hierarchy $\left(m_{3} \ll m_{1}, m_{2}\right)$ the minimal summed mass is $0.1 \mathrm{eV}$. The individual masses in these hierarchical limits are well below the detection limit of current and future laboratory $\beta$-decay experiments, but can be probed by cosmology. Massive neutrinos suppress gravitational clustering on scales below the horizon size at the non-relativistic transition, reducing the lensing power spectrum (as well as galaxy clusters number counts - see section 3). We illustrate the capabilities of PRISM to distinguish the minimal-mass inverted hierarchy from a model with three massless neutrinos via its lensing power spectrum in figure 10. Combining the anisotropy and lensing power spectra of PRISM, we forecast a $1 \sigma$ error of $0.04 \mathrm{eV}$ for the summed mass, comparable to the hierarchical target masses. This constraint can be improved further by combining with near-future BAO measurements, for example by a factor of two using BOSS, at which point it becomes possible to distinguish between the normal and inverted hierarchies (in the hierarchical limits) [83].

The cosmological constraints from lensing with PRISM would be highly complementary to those from upcoming cosmic shear surveys in the optical, such as Euclid. For example, the systematic effects are quite different with non-linearities being much less of an issue for CMB lensing with its peak sensitivity to large-scale structures around $z=2$. Moreover, there are no intrinsic alignments of galaxy ellipticities to worry about. The combination of 


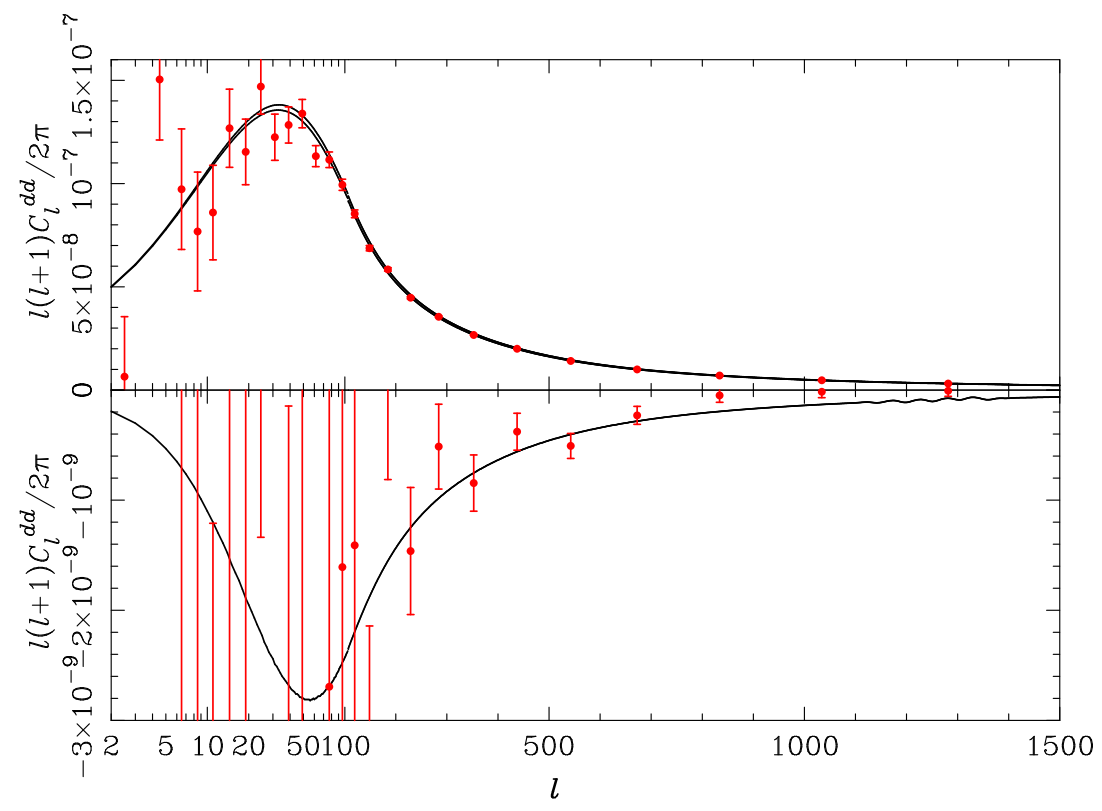

Figure 10. Simulated deflection power spectrum from PRISM assuming a minimal-mass inverted hierarchy $\left(\sum m_{\nu} \approx 0.1 \mathrm{eV}\right)$. In the upper panel, the solid lines are the theory power spectrum for this scenario (lower) and for three massless neutrinos (upper). The difference between these spectra is plotted in the lower panel illustrating how PRISM can distinguish these scenarios with its lensing power spectrum in the range $\ell>200$.

the two probes of mass is particularly promising, since it allows calibration of multiplicative bias effects such as due to PSF corrections in the optical (e.g. ref. [208]).

The high $S / N$ reconstruction of the lensing potential by PRISM is illustrated in figure 11. The left panel is a simulated map of the filtered lensing potential (in Fourier space, the image is $\ell \phi(\ell)$ with $\phi(\ell)$ the Fourier transform of the lensing potential, so has the same dimensions as the deflection field) over a small patch of the sky. The middle and right panels illustrate the fidelity of the lensing reconstructions expected from the full Planck mission and PRISM, respectively. A similar quality reconstruction will be obtained with PRISM over nearly the full sky.

Cross-correlating CMB lensing with other probes of large-scale structure, such as galaxies $[20,79,94,160,193,195]$, the Ly $\alpha$ forest [209] or CIB clustering [85, 95, 161] (see also section 4), also has exceptional promise, allowing self-calibration of the tracer's bias relation at the sub-percent level. Such cross-correlations with tracers selected as a function of redshift will make possible the three-dimensional characterization of the lensing potential, and hence the reconstruction of the distribution of matter inhomogeneities in three dimensions over most of our Hubble volume. A combination of this with the bulk-velocity constraints obtained from $\mathrm{kSZ}$ observations will give an unprecedented view of cosmic structure formation in the entire observable Universe.

\subsection{Primordial non-Gaussianity}

With the first Planck cosmology data release, non-Gaussianity (NG) crossed a significant threshold to become a robust quantitative probe of cosmological physics [165]. Planck results dramatically improved previous NG analyses, offering the most stringent test to date of inflationary theory ( with $f_{\mathrm{NL}}^{\text {loc }}=2.7 \pm 5.8$ ) while also detecting for the first time ISW-lensing 
Input

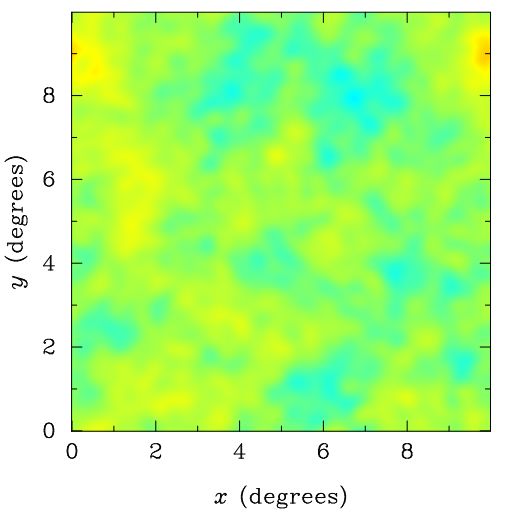

Planck (simulation)

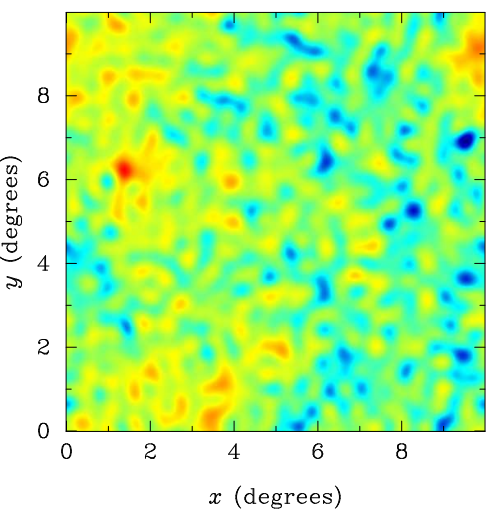

PRISM

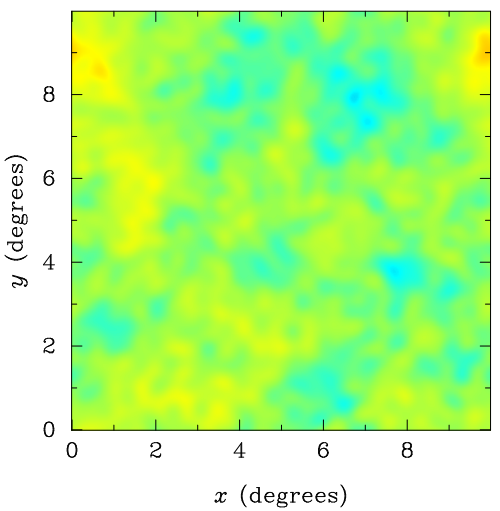

Figure 11. Reconstruction of the $\mathrm{CMB}$ lensing potential. Right: $10^{\circ} \times 10^{\circ}$ simulated map of the (filtered) lensing potential for a standard $\Lambda$ CDM cosmology matching Planck cosmological parameter constraints. Middle: simulated reconstruction of the lensing potential on the same field with the sensitivity of the full Planck mission. Right: simulated reconstruction with the PRISM sensitivity. The same color scale is used for all three maps.

and diffuse point source bispectra. Already Planck offers enticing clues about the nontrivial 'shape' of the CMB bispectrum of our universe (as illustrated in figure 12), the origin of which is yet to be explained. PRISM would offer the highest precision reconstructions of the CMB temperature and polarization bispectra and trispectra which, in conjunction with the CMB power spectra, will provide a decisive and unambiguous probe of primordial cosmology back to the Planck era. At the same time, PRISM NG data will open new windows for investigating dark energy, gravitational physics, and cosmological parameters, as well as astrophysical sources, large-scale structure and galactic history.

An important and unique advantage of PRISM over non-CMB probes of NG results from its ability to recognize the distinct patterns that physical mechanisms leave in the shape of higher-order correlators, as illustrated in figure 13. The competitive strength of PRISM will therefore be a vastly enhanced exploration of physically-predicted NG shapes compared to any other projected probe of NG. While other probes claim competitive power for detecting 'local' bispectral non-Gaussianity, these claims do not yet address the full suite of non-linear systematic and biasing effects.

Only a high-resolution polarization CMB mission such as PRISM can provide the high sensitivity for detecting and distinguishing differing NG bispectral shapes. In fact, polarization maps contain more information than temperature maps and the best constraints arise from combining the two. Polarization is the more powerful probe because the ratio of the expected contaminant signal (mainly Galactic dust polarization) to the primordial signal is smaller for polarization than for temperature for the majority of resolved modes. To construct a figure of merit comparing the predicted impact of PRISM on physical forms of NG, we compare the predicted constraint volume in bispectrum space spanned by the local, equilateral and flattened bispectra. Compared to WMAP data, the first Planck release reduced the constraint volume by a factor of 20 and full-mission Planck data including polarization is expected to improve this by an additional factor of 2.5; PRISM would go beyond this by another factor of 30. Considering the constraint volume based only on the polarization maps (which provide information independent of the temperature maps and hence provide an important consistency check), we find a volume reduction factor from Planck to PRISM 


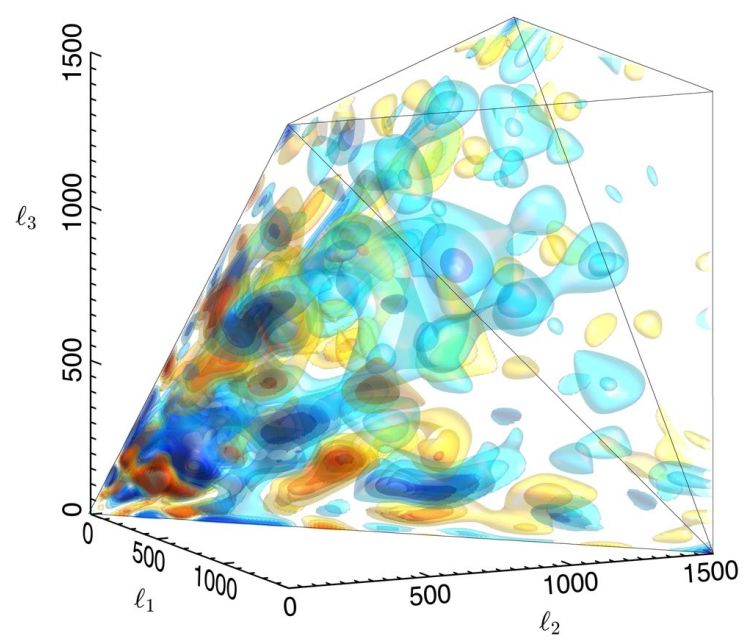

Figure 12. Planck CMB temperature (TTT) bispectrum [165]. The bispectrum depends on three multipoles $\ell_{1}, \ell_{2}, \ell_{3}$ subject to a triangle constraint, so it contains rich $3 \mathrm{D}$ shape information in a tetrahedral domain. Isocontours are plotted with red positive and blue negative. Note the periodic CMB ISW-lensing signal in the squeezed limit along the edges (seen at about the $2.5 \sigma$ level). PRISM would detect this signal at $9 \sigma-$ a unique window on dark energy. Scale-invariant signals predicted by many inflationary models are strongly constrained by this data though 'oscillatory' and 'flattened' features hint at new physics. PRISM will probe these hints with an order of magnitude more resolved triangle configurations as well as polarization cross-terms.

of order 110. PRISM would therefore greatly advance unambiguous precision tests of the standard inflationary paradigm. will be distinct from other experiments because of its fullsky coverage, polarization sensitivity, high resolution, strong rejection of systematics, and the benign environment in space at L2 making PRISM the ideal CMB NG probe.

The improvement compared with Planck will be even more dramatic when considering combined bispectrum and trispectrum constraints on a larger range of NG shapes. The forecast precision with which local trispectrum parameters could be measured with PRISM are $\Delta g_{\mathrm{NL}}=3 \times 10^{4}$ and $\Delta \tau_{\mathrm{NL}}=1 \times 10^{2}$ [194]. These could investigate consistency conditions between trispectra and bispectra, which can be used to test large classes of multi-field inflation models, in addition to single-field inflation. There are other alternative inflationary scenarios for which an observable non-Gaussian signal is quite natural, including models with non-canonical kinetic energy, additional vector fields, features or periodicity in the inflationary potential, or remnants of a pre-inflationary phase. There are also more exotic paradigms which can create NG such as cosmic (super-)strings [166] or a contracting phase with a subsequent bounce [121]. Each of these models has a distinct fingerprint, many uncorrelated with the standard three primordial shapes and, in all cases, PRISM would significantly improve over present Planck constraints, offering genuine discovery potential. Beyond searches for primordial NG, PRISM is guaranteed to make important observations of late-time NG. For example, PRISM will decisively detect and characterize the lensing-ISW correlation, driven by dark energy, achieving a $9 \sigma$ detection, resulting in a new probe of dark energy physics from the CMB alone. Second-order recombination effects can also be probed; present calculations (temperature only) suggest PRISM could detect these at about $3 \sigma[114,198]$. Furthermore, ancillary signatures will also constrain modified gravity alternatives to the standard cosmological model. 

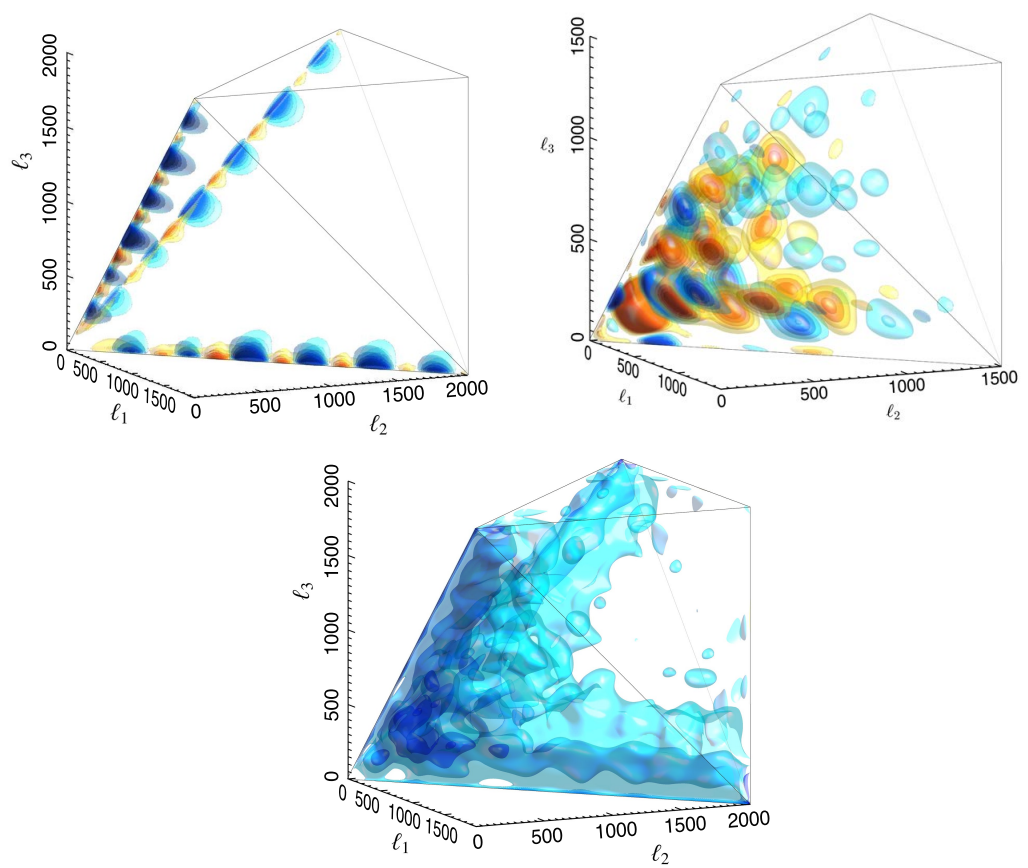

Figure 13. Primordial and late-time non-Gaussian shapes $[165,166]$. Here the distinct CMB bispectra $B_{l_{1} l_{2} l_{3}}$ from three theoretical models are plotted (from left to right): the ISW-lensing signal (observed in figure 12); an inflationary 'feature' model; and the late-time bispectrum generated by cosmic strings.

CMB spectral distortions (further discussed in section 7) can also open up a new window through which to search for primordial NG [148]. We know almost nothing about NG on the small scales that can be probed via these observations. In particular the 2-point cross-correlation between $\mu$-type distortions and CMB anisotropies turns out to be naturally sensitive to the very squeezed limit of the primordial bispectrum (probing scales as small as $50 \leq k \mathrm{Mpc} \leq 10^{4}$ ). Also, the power spectrum of $\mu$-distortions can probe the trispectrum of primordial fluctuations. Such measurements can be particularly constraining for models where the power spectrum grows on small scales (see, e.g. ref. [37]). Values as low as $f_{\mathrm{NL}}^{\text {loc }}<1$ can be achieved. Also, $\mu$-type distortions can shed light into modifications of the initial state of quantum fluctuations. For a large class of inflationary models characterized by a non-Bunch-Davies vacuum (whose bispectrum is enhanced in the squeezed limit with respect to the local form) a high $S / N$ can be achieved [78]. Being sensitive to small scales, the cross-correlation between $\mu$-type distortions and CMB anisotropies can also be employed to probe primordial NG that runs with scales [16].

\subsection{Parameters from high-resolution polarization spectra}

PRISM will measure the CMB temperature and polarization angular power spectra with outstanding precision to small angular scales. Combining the five channels in the $105-200 \mathrm{GHz}$ frequency range, the $T T$ spectrum is cosmic-variance limited to $\ell=3850$ (figure 8). Although uncertainties in foreground modelling, in particular the background of radio and infrared sources, might limit the maximum useful multipole to lower values, the large frequency coverage of PRISM will help correct for such contamination in the best possible way. 

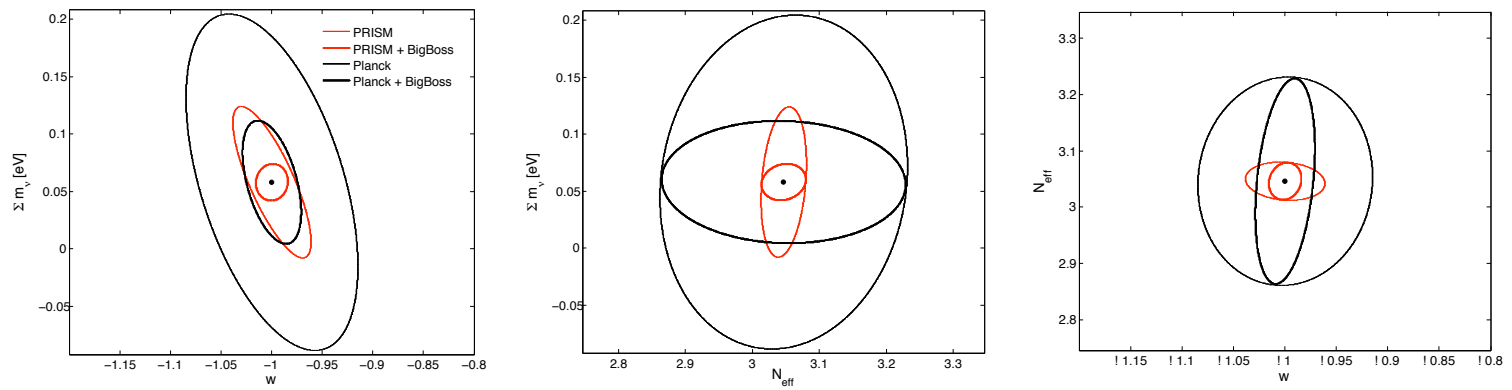

Figure 14. Fisher forecasts for the $68 \%$ confidence regions in 10-parameter models (the standard six parameters plus the summed neutrino mass $m_{\nu}$, effective number of relativistic degrees of freedom $N_{\text {eff }}$, equation of state of dark energy $w$, and spatial curvature). 2D marginalised constraints are shown in the $\sum m_{\nu}-w$ plane (left), $\sum m_{\nu}-N_{\text {eff }}$ (middle), and $N_{\text {eff }}-w$ (right) for the full-mission Planck data including lens reconstruction (thin black lines) and further combining with BAO data from BigBoss/DESI (thick black lines). Results for PRISM are shown as thin red contours, and for PRISM+BigBoss in thick red.

As noted above however, high- $\ell$ polarization is expected to be cleaner, and with PRISM the $E E$ spectrum is cosmic-variance limited to $\ell=2500$ (and the $B$-mode power from lensing to $\ell=1100) .{ }^{3}$ Such a remarkable measurement of the polarization of the CMB damping tail will be an invaluable source of information for several reasons. Ongoing experiments, such as Planck have confirmed that the tail of the $T T$ power spectrum can not only constrain inflationary models by providing a long lever-arm for measuring the shape of the power spectrum of the primordial curvature perturbation, but also provide detailed information about the fundamental matter content of the Universe. This includes the effective number of relativistic species $N_{\text {eff }}$, for which a non-standard value (as hinted by pre-Planck anisotropy measurements) could be due to sterile neutrinos, as advocated in particle physics to explain certain anomalies in the neutrino sector, the helium abundance $Y_{\mathrm{P}}$, which provides a clean test of standard big-bang nucleosynthesis, and the neutrino mass. Measuring the high- $\ell$ damping tail in polarization will allow us to place strong constraints on a number of different models, free from the foreground issues that limit inferences made from the temperature measurements.

In order to illustrate the potential improvements that PRISM will bring, we have made MCMC parameter forecasts, following ref. [77]. In $\Lambda$ CDM models, the spectral index and its running will be measured more precisely than with current Planck data by factors of five and three, respectively. The Hubble constant (a point of tension between Planck data and direct astrophysical measurements) will be measured a factor of 10 better than currently (and 2.5 times better than expected from the full Planck data). In one-parameter extensions to the matter content beyond $\Lambda \mathrm{CDM}$, we forecast that PRISM will measure $N_{\text {eff }}$ to $2 \%$ precision and $Y_{\mathrm{P}}$ to $1 \%$. These values indicate that a $2 \sigma$ anomaly hinted at by Planck could be confirmed decisively with PRISM. Moreover, from its measurement of the $B$-mode power spectrum, PRISM should extend the range of sensitivity to cosmic strings by an order of magnitude over the recent Planck constraints [2, 166].

The combination of temperature and polarization power spectrum measurements to small angular scales and lensing reconstruction with PRISM will allow precise exploration

\footnotetext{
${ }^{3}$ The current Planck TT power spectrum is cosmic-variance limited for $\ell \lesssim 1500$, while forthcoming Planck polarization data is expected to be noise-dominated for nearly all multipoles.
} 
of more complex models. For example, models in which an extended neutrino sector, e.g. including the mass of the active neutrinos as well as additional massless species (parametrised by $N_{\text {eff }}$ ), is combined with evolving dark energy (with equation-of-state parameter $w$ ) and spatial curvature. In such complex models, parameter degeneracies are significant for current CMB data and adding external data, such as BAO, leads to large improvements in statistical errors. Forecasts based on the Fisher information ${ }^{4}$ for such a 10-parameter model are shown in figure 14 for the full Planck mission (including lens reconstruction and rather less conservative sky fractions than adopted for the 2013 temperature analysis), full Planck combined with next-generation BAO measurements from BigBOSS/DESI, PRISM (including lens reconstruction), and PRISM combined with BigBOSS/DESI. Significantly, PRISM alone is almost as powerful for the late-time parameters $\sum m_{\nu}$ and $w$ as the combination of the full Planck mission and next-generation BAO measurements, and is much more powerful for early-time parameters such as $N_{\text {eff }}$. The combination of PRISM and next-generation BAO measurements is particularly powerful, improving over full Planck plus next-generation BAO by a factor of three on the (summed) neutrino mass and factors of two for $w$, the curvature parameter and $N_{\text {eff }}$. With this data combination, constraints on the absolute mass scale of neutrinos at the $0.02 \mathrm{eV}$ are robust to extensions to the dark energy model and the spatial geometry.

\section{CMB spectral distortions}

The COBE FIRAS instrument established that the average CMB spectrum is extremely close to a perfect blackbody, with possible departures limited to $\Delta I_{\nu} / I_{\nu} \lesssim$ few $\times 10^{-5}[74,133]$. Similarly, the tiny directional variations of the CMB intensity and polarization, imprinted by perturbations of the photon-baryon fluid at the last scattering surface, all have a thermal spectrum $[13,159,197]$. This observation places very tight constraints on the thermal history of our universe, ruling out cosmologies with extended periods of significant energy release at redshifts $z \lesssim$ few $\times 10^{6}[26,33,43,56,98,104,110,200,219]$. However a large number of astrophysical and cosmological processes cause inevitable spectral distortions of the CMB at a level detectable using present day technology. The measure of the absolute spectrum by PRISM will open an unexplored window to the early universe, allowing detailed studies of many expected or possible processes of energy injection throughout cosmic history (figure 15), targeting several sources of early energy release (figure 16).

\subsection{Reionization and structure formation}

Radiation from the first stars and galaxies [4, 97], feedback by supernovae [146] and structure formation shocks $[30,139,201]$ heat the IGM at redshifts $z \lesssim 10-20$, producing hot electrons that up-scatter CMB photons, giving rise to a Compton $y$-distortion with average amplitude $\Delta I_{\nu} / I_{\nu} \approx 10^{-7}-10^{-6}$. This signal will be detected at more than a $100 \sigma$ with PRISM, providing a sensitive probe of reionization physics and delivering a census of the missing baryons in the local Universe. PRISM furthermore has the potential to separate the spatially varying signature caused by the WHIM and proto-clusters [181, 220]. It also offers a unique opportunity to observe the free-free distortion associated with reionization [172]. The amplitude of the signal depends on the clumping of matter and thus provides complementary way to study the late evolution of inhomogeneities.

\footnotetext{
${ }^{4}$ In models with near degeneracies, Fisher forecasts are unreliable. The relative improvements in the constraints plotted in figure 14 are more accurate than the absolute forecasted errors.
} 


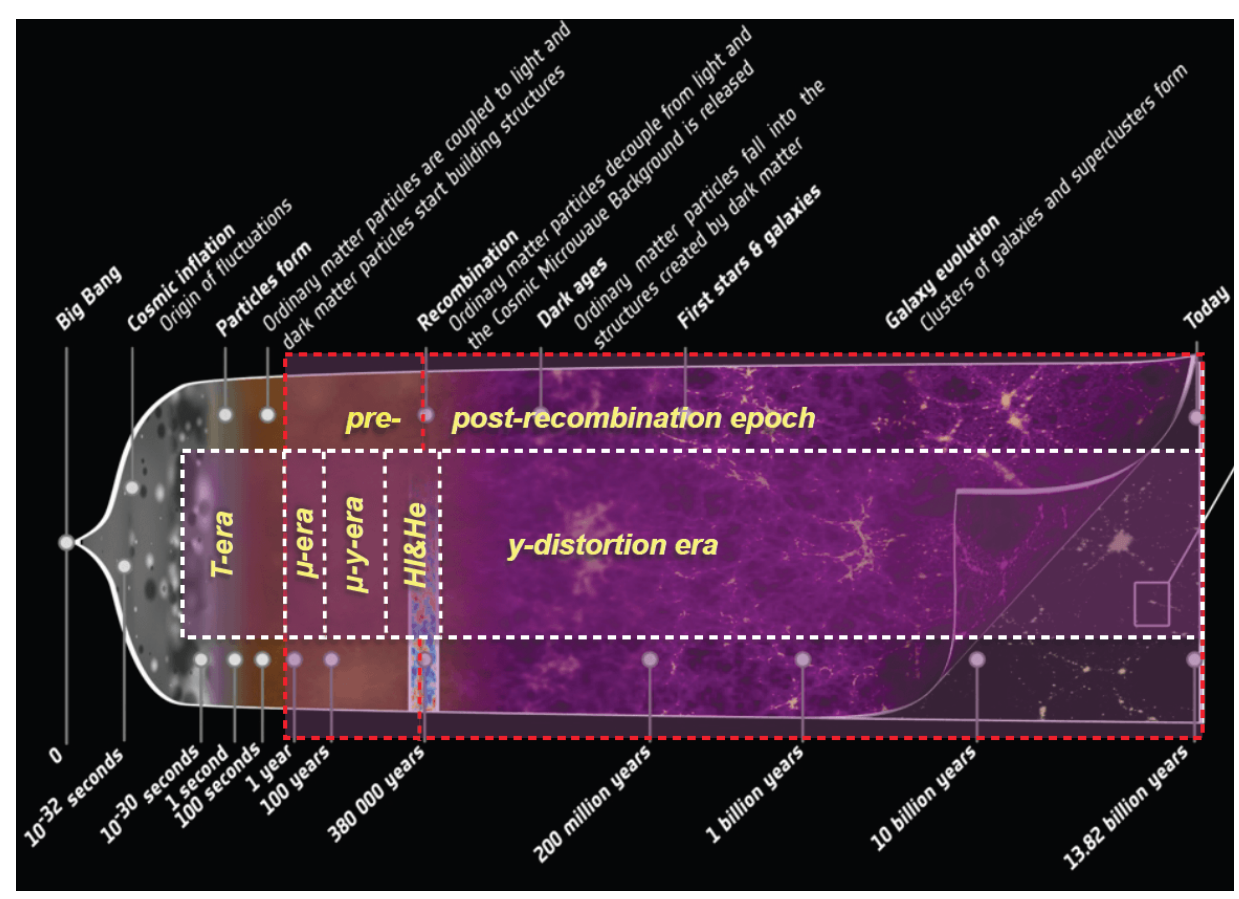

Figure 15. CMB spectral distortions provide a sensitive probe of energy release throughout the cosmic history. At very early stages $\left(z \gtrsim\right.$ few $\left.\times 10^{6}\right)$, thermalization is extremely efficient and only a shift in the average CMB temperature is produced, leaving an unmeasurable distortion signature. At later stages $\left(3 \times 10^{5} \lesssim z \lesssim\right.$ few $\left.\times 10^{6}\right)$, when the Universe was only a few months old, energy release causes a pure $\mu$-distortion. This type of distortion is unique to the early Universe and thus tells a story about processes occurring deep in the pre-recombination epoch. At redshift $z \lesssim 10^{4}$, on the other hand, a pure Compton $y$-distortion is created, providing the means to learn about reionization and structure formation. At intermediate stages $\left(10^{4} \lesssim z \lesssim 3 \times 10^{5}\right)$, the distortion interpolates between $\mu$ - and $y$-distortion, providing additional information about the time dependence of the energy release scenario encoded in the smaller residual (non- $\mu /$ non- $y$ ) distortion. PRISM will target this signal, which will inform us about decaying particles and the small-scale power spectrum, observations that would otherwise be impossible. The hydrogen and helium recombination lines, which are created at redshifts $z \approx 10^{3}$, might furthermore allow PRISM to distinguish pre- and post-recombination $y$-distortions.

\subsection{Constraining the inflaton}

Silk damping of small-scale perturbations of the photon fluid gives rise to CMB distortions $[5,54,96,200]$ that depend directly on the shape and amplitude of the primordial power spectrum at scales $0.6 \mathrm{kpc} \lesssim \lambda \lesssim 1 \mathrm{Mpc}$ (or multipoles $10^{5} \lesssim \ell \lesssim 10^{8}$ ) [40, 113] (see figure 17 and 18 for illustration). This allows constraining the trajectory of the inflaton at stages unexplored by ongoing or planned experiments [37, 39, 111, 174], extending our reach from 7 e-folds of inflation probed with the CMB anisotropies to a total of 17 e-folds (see figure 19 for detection limits). The signal is also sensitive to the difference between adiabatic and isocurvature perturbations $[5,38,63,100]$, as well as primordial non-Gaussianity in the ultra squeezed-limit, leading to a spatially varying spectral signal that correlates with CMB temperature anisotropies as large angular scales [78, 148]. This effect therefore provides a unique way to study the scale-dependence of $f_{\mathrm{NL}}[16]$. CMB spectral distortions thus provide a complementary and independent probe of early-Universe physics, with PRISM capitalizing on the synergies with large-scale B-mode polarization measurements. 

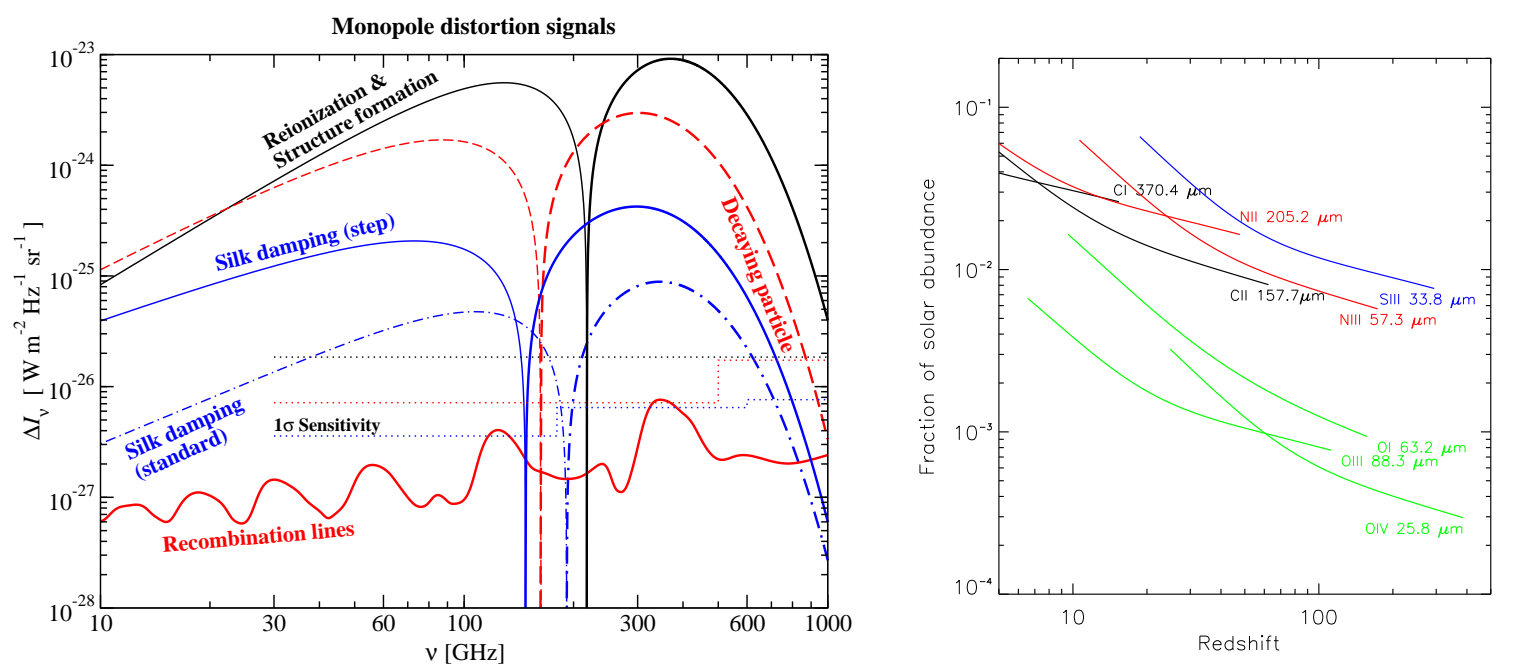

Figure 16. Left: spectral distortions for different scenarios. Thick lines denote positive, and thinner lines negative signal. The $1 \sigma$ sensitivities of PRISM for different designs are also indicated. The largest distortion is from the heating of the medium during reionization and structure formation, here for $y \approx 5 \times 10^{-7}$. The decaying particle scenario is for lifetime $t_{\mathrm{X}} \approx 3.6 \times 10^{9}$ sec and total energy release $\Delta \rho_{\gamma} / \rho_{\gamma} \approx 6.3 \times 10^{-7}$. Two cases for the distortion caused by dissipation of smallscale curvature perturbations are shown, one for the standard power spectrum, extrapolated from large-scale CMB measurements all the way to $k \approx$ few $\times 10^{4} \mathrm{Mpc}^{-1}$, the other assuming an additional step $\Delta A_{\zeta}=10^{-8}$ at $k=30 \mathrm{Mpc}^{-1}$ in the power spectrum. The signal caused by recombinations of hydrogen and helium is also, in principle, directly detectable (combining adjacent frequencies). Right: projected constraints on the abundance of different metal ions versus redshift assuming inter-channel calibration accuracy of $\approx 10^{-5}$.

\subsection{Decaying and annihilating relics}

The CMB spectrum allows placing tight limits on decaying and annihilating particles during the pre-recombination epoch $[34,43,99,134]$. This is especially interesting for decaying particles with lifetimes $t_{\mathrm{X}} \approx$ few $\times 10^{8} \mathrm{sec}-10^{11} \mathrm{sec}[35,39]$, as the exact shape of the distortion encodes when the decay occurred [36, 43, 109]. Decays or annihilations associated with significant low-energy photon production furthermore create a unique spectral signature that can be distinguished from simple energy release [55, 98] (see figure 19). PRISM therefore provides an unprecedented probe of early-universe particle physics (e.g., dark matter in excited states [73, 173] and Sommerfeld-enhanced annihilations close to resonance [84]), with many natural particle candidates found in supersymmetric models [71, 72].

\subsection{Metals during the dark ages}

Any scattering of CMB photons after recombination blurs CMB anisotropies at small scales, while producing new anisotropies at large scales. Electrons from the reionization epoch are the dominant source of optical depth, causing a frequency-independent signature already detected by WMAP and Planck [13, 159]. The resonant scattering of CMB photon by fine structure lines of metals and heavy ions produced by the first stars adds to this optical depth, making it frequency-dependent $[10,128]$. By comparing CMB temperature and polarization anisotropies at different frequencies one can thus determine the abundances of ions such as OI, OIII, NII, NIII, CI, CII at different redshifts [89, 91]. Furthermore, UV radiation emitted by the first stars can push the OI $63.2 \mu \mathrm{m}$ and CII $157.7 \mu \mathrm{m}$ transitions out of equilibrium with 


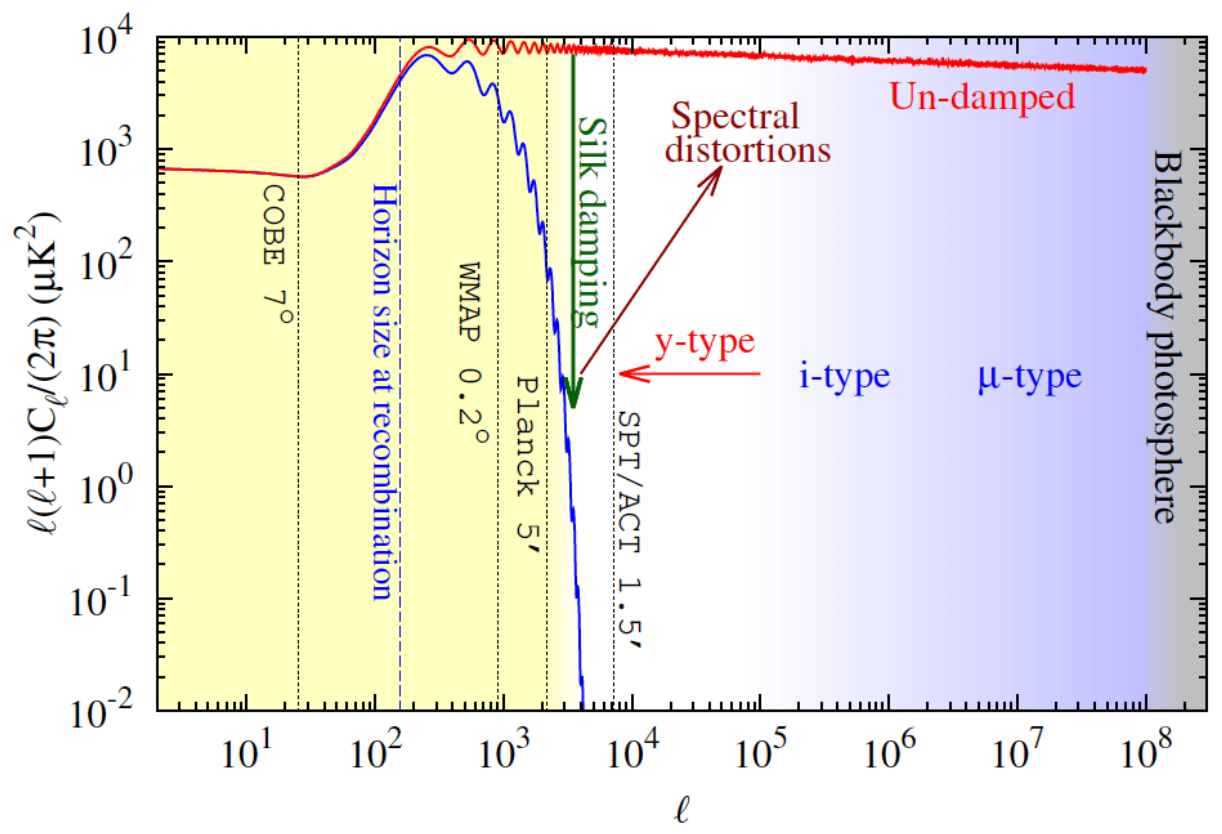

Figure 17. Silk damping erases primordial anisotropies on small scales from the CMB, and current experiments have reached this resolution limit set by damping. CMB spectral distortions allow us to bypass this limit and extend our knowledge of initial conditions by many orders of magnitude in scale, complementing CMB anisotropy experiments. CMB spectral distortion measurements provides information that could be otherwise obtained (in the absence of damping) with a conventional anisotropy experiment with the extreme angular resolution of 6 milli-arcsec. This figure is adapted from [112].
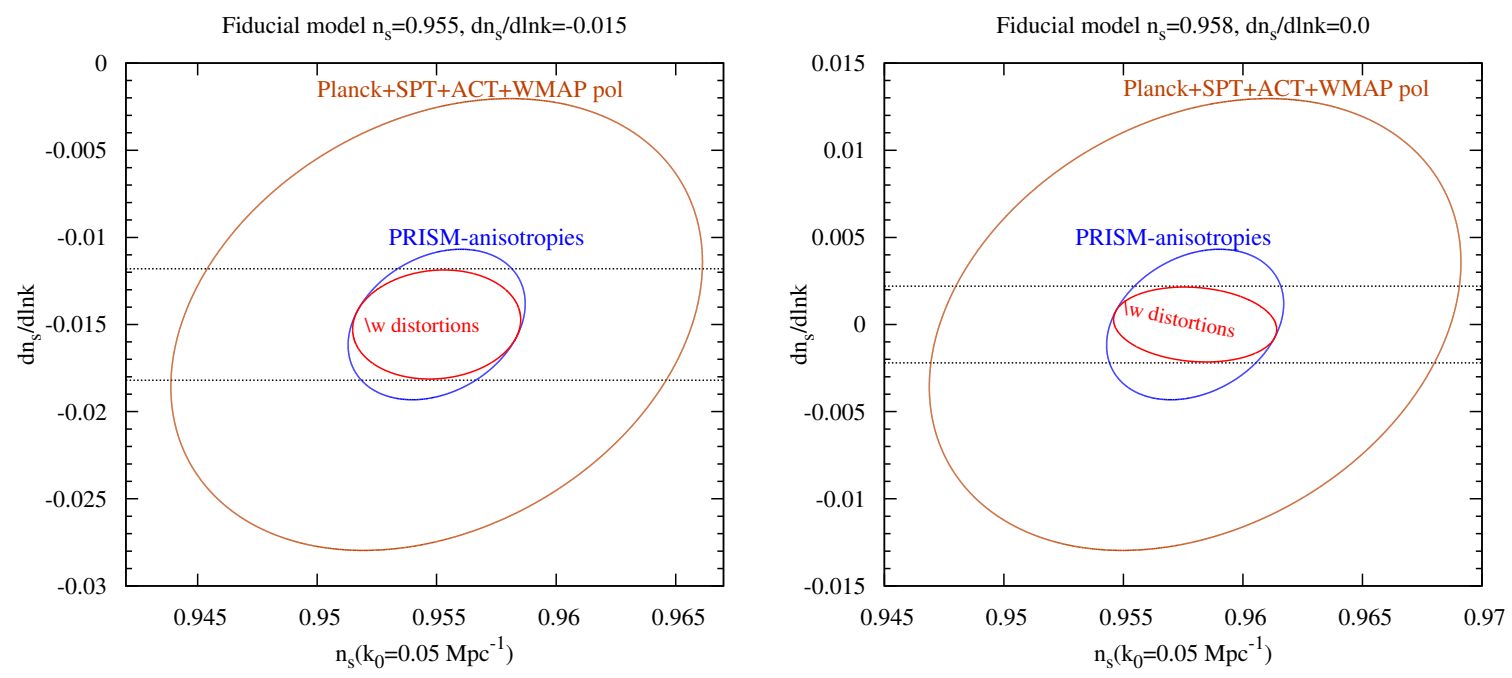

Figure 18. Fisher forecasts for constraints on the primordial power spectrum from PRISM for a power spectrum with (left panel) and without (right panel) running [111, was used for the computation]. Combined power of anisotropies and CMB spectral distortions will nail down the running of power spectrum to about \pm 0.002 providing stringent tests of single-field inflation $[69,126]$, significantly improving the constraints from current $\mathrm{CMB}$ experiments. 

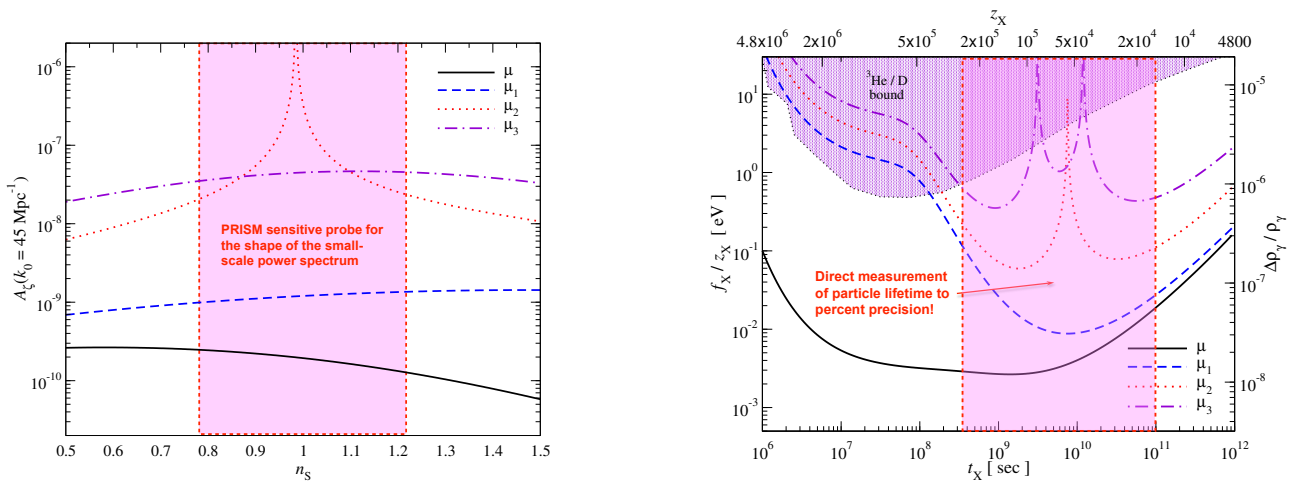

Figure 19. PRISM will provide a unique probe of the small-scale power spectrum (top panel) and decaying particles (bottom panel). Here we show the $2 \sigma$-detection limits deduced from the $\mu$-parameter and the first few distortion principle components, $\mu_{i}$, [see 39, for details]. The extra information contained in the residual (non- $\mu /$ non- $y$ ) distortion will independently constrain the amplitude and spectral index of the primordial power spectrum around pivot scale $k \approx 45 \mathrm{Mpc}^{-1}$, superseding the constraint deduced from ultracompact minihalos or black hole abundances [23] by at least 2 orders of magnitude. The signatures of decaying particles will furthermore independently constrain their abundance (parameterized by $f_{\mathrm{X}}$ ) and lifetime, $t_{\mathrm{X}}$, with highest sensitivity to particles with $t_{\mathrm{X}} \approx$ few $\times 10^{8} \mathrm{sec}-10^{11} \mathrm{sec}$. PRISM will improve existing limits from light elements [we show those adapted from figure 42 of 108] by some 2 orders of magnitude. These figures are adapted from [39].

the CMB, producing a distortion $\Delta I_{\nu} / I_{\nu} \approx 10^{-8}-10^{-9}$ due to fine structure emission [81, 88], providing yet another window within the reach of PRISM for probing reionization.

\subsection{Cosmological recombination radiation}

The recombination of $\mathrm{H}$ and He introduces distortions $[67,150,218]$ at redshifts $z \approx 10^{3}-$ $10^{4}$, corresponding to $\approx 260 \mathrm{kyr}(\mathrm{H} \mathrm{I}), \approx 130 \mathrm{kyr}(\mathrm{HEI})$, and $\approx 18 \mathrm{kyr}$ (HE II) after the big bang $[41,187,188]$. The signal is small $\left(\Delta I_{\nu} / I_{\nu} \approx 10^{-9}\right)$ but its unique spectral features promise an independent determination of cosmological parameters (such as the baryon density and pre-stellar helium abundance) and direct measurements of recombination dynamics, probing the Universe at stages well before the last scattering surface [199].

At the highest sensitivity observing mode, PRISM can directly detect the $\mathrm{H} \alpha$ and $\mathrm{P} \alpha$ lines. Furthermore, if something unexpected happened during different stages of the recombination epoch, atomic species will respond $[42,131]$ by producing additional distortion signals that can exceed those of the normal recombination process. This behavior provides a unique way to distinguish pre- from post-recombination energy release [34, 42].

\subsection{Hydrogen line scattering}

Another interesting signature arises from resonant scattering of CMB photons by the hydrogen lines during recombination, introducing a frequency-dependent optical depth contribution and hence a CMB anisotropy signal $[89,90,189]$. The effect on the TT power spectrum from the first lines of the Balmer and Paschen series is detectable with PRISM and provides an additional opportunity to directly constrain the recombination history and obtain independent determinations of cosmological parameters (e.g. $\Omega_{\mathrm{b}}$ or $\Omega_{\mathrm{m}}$ ). Figure 20 shows the predicted signal for the $\mathrm{H} \alpha$ line, with a maximum amplitude of $\approx 0.3 \mu \mathrm{K}$ at frequencies of $\approx 450 \mathrm{GHz}$ and at small angular scales corresponding to $\ell \approx 870$. For the $\mathrm{P} \alpha$ line, the intensity reaches $\approx 0.02 \mu \mathrm{K}$ at $\approx 150 \mathrm{GHz}$ and the same angular scales. 


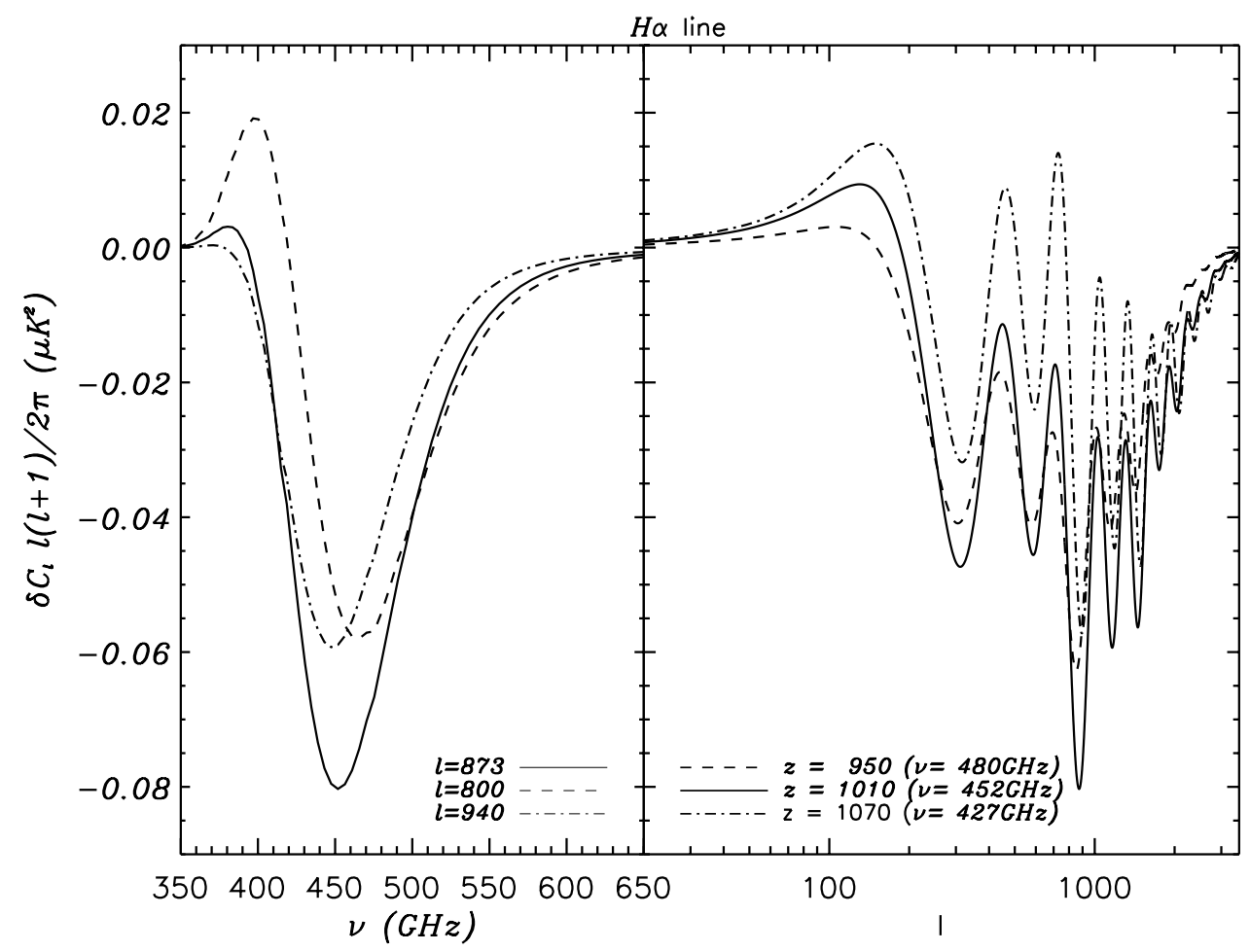

Figure 20. Change in the CMB angular power spectrum (TT) arising from the scattering of photons in the $\mathrm{H} \alpha$ line during recombination. The left panel illustrates the signal as a function of the redshifted frequency, while the right panel shows the angular multipole. Solid lines in both panels refer to the cases in which the signal is largest.

\subsection{Rayleigh scattering}

Neutral hydrogen produced as the Universe recombines is not completely transparent to the CMB. Rayleigh scattering by atomic hydrogen modifies the primary CMB anisotropies in both temperature and polarization by providing additional damping on small scales and also producing new temperature and polarization signals [123, 216]. PRISM will measure the amplitude of temperature signal from Rayleigh scattering with $0.4 \%$ accuracy and EE polarization signal at $\approx 20 \sigma$. The part of the Rayleigh signal correlated with primary CMB will constrain the recombination history. The uncorrelated part will probe 10,000 new modes at $\ell<500$ [123]. Because of its $\sim \nu^{4}$ characteristic frequency dependence, the signal can be distinguished from the resonant scattering by the $\mathrm{H} \alpha$ line [189]. The detection of the frequency dependent signals, even on large scales, is not limited by cosmic variance but by sensitivity and foregrounds. These signals are therefore ideal targets for PRISM.

\subsection{Cooling of matter}

The adiabatic cooling of ordinary matter continuously extracts energy from the CMB photon bath, leading to a small but certain distortion characterized by a negative chemical potential and $y$-parameter at the level of $\approx$ few $\times 10^{-9}[33,43,112]$. PRISM will be sensitive to this signal, delivering an independent way to determine the baryon density. 


\subsection{Cosmic strings and primordial black holes}

Cosmic strings arise naturally in many proposed theories of new physics beyond the standard model unifying the electroweak and strong interactions as well as in many superstring inspired inflation models. If the cosmic strings are superconducting cosmic strings, they would also produce copious electromagnetic radiation, creating CMB spectral distortion of a unique shape distinguishable from other sources of distortions if detected at high $\mathrm{S} / \mathrm{N}[147,203,204]$. Evaporating primordial black holes provide another source of energy injection, with the shape of the resulting distortion depending on the black hole mass function [29]. For nonevaporating black holes, CMB spectral distortions could furthermore be used to constrain the black hole spin [149]. PRISM is sensitive to a rich variety of new physics and will in any case provide constraints on physics beyond the standard model.

\subsection{PRISM's discovery potential using spectral distortion}

The above examples demonstrate that the CMB spectrum provides a rich and unique source of complementary information about the early Universe, with several predicted spectral distortions within the reach of PRISM. But the science is not limited to this. The CMB spectrum could also place interesting constraints on the power spectrum of small-scale magnetic fields [107], decay of vacuum energy density [7, 25, 55], axions [205] and other new physics examples $[22,24,28,127,210]$. Deciphering these signals is a challenge, but holds the potential for important new discoveries and constraining unexplored processes that cannot be probed by other means.

\section{Structure of the dusty magnetized Galactic ISM}

The data analysis is still on-going but it is already clear that Herschel and Planck will have a profound and lasting impact on our understanding of the interstellar medium and star formation. PRISM holds even greater promise for breakthroughs. Dust and synchrotron radiation are the dominant contributions to the sky emission and polarization to be observed by PRISM. Dust emission is an optically thin tracer of the structure of interstellar matter. Synchrotron radiation traces the magnetic field over the whole volume of the Galaxy, while dust polarization traces the magnetic field within the thin star forming disk, where the interstellar matter is concentrated. PRISM will image these two complementary tracers with unprecedented sensitivity and angular resolution. It will also provide all-sky images of spectral lines, which are key diagnostics of interstellar gas physics. No other initiative offers a comparable imaging capability of interstellar components over as wide a range of scales. In the following subsections we detail how PRISM will address three fundamental questions of Galactic astrophysics: (1) What are the processes that structure the interstellar medium? (2) What role does the magnetic field play in star formation? (3) What are the processes that determine the composition and evolution of interstellar dust?

\subsection{Structure of interstellar medium}

Herschel far infrared observations have provided astronomers new insight into how turbulence stirs up the interstellar gas, giving rise to a filamentary, web-like structure within the diffuse interstellar medium and molecular clouds. PRISM will extend the Herschel dust observations to the whole sky and provide unique data on emission lines key to quantifying physical processes. The spectral range of PRISM includes atomic and molecular lines that serve 
as diagnostics of the gas density and temperature, its chemical state, and energy budget. Herschel has observed these lines along discrete lines of sight with very limited imaging. By mapping these lines and dust emission over the whole sky at an angular resolution comparable to that of Herschel, PRISM will probe the connection between the structure of matter and gas cooling across scales.

The PRISM sky maps will provide multiple clues to characterize the physical processes that shape interstellar matter. The CII, CI, and OI fine structure lines and the rotational lines of $\mathrm{CO}$ and $\mathrm{H}_{2} \mathrm{O}$ are the main cooling lines of the cold neutral interstellar medium and of molecular clouds. These lines probe local physical conditions and the exchange of energy associated with the formation of molecular gas within the diffuse interstellar medium and of stars within molecular clouds. The NII lines at 122 and $205 \mu \mathrm{m}$ are spectroscopic tracers of the ionized gas. These lines are essential for distinguishing the contribution of neutral and ionized gas to the CII emission. PRISM will have the sensitivity to image the CII line emission at sub-arcminute resolution even at the Galactic poles. The CII map can be combined with HI and dust observations to study the formation of cold gas from the warm neutral medium through the thermal instability. This analysis will probe the expected link, yet to be confirmed observationally, between the small-scale structure of the cold interstellar medium and gas cooling. The CII line emission is also key to studying the formation of molecular gas by tracing the CO-dark $\mathrm{H}_{2}$ gas [154]. In star forming molecular clouds, the $\mathrm{CO}, \mathrm{CI}, \mathrm{OI}$, and $\mathrm{H}_{2} \mathrm{O}$ lines are the key tracers of the processes creating the initial conditions of star formation and of the feedback from newly formed stars on their parent clouds.

\subsection{Galactic magnetic field and star formation}

Star formation results from the action of gravity, counteracted by thermal, magnetic, and turbulent pressures [87]. For stars to form, gravity must locally become the dominant force. This happens when the turbulent energy has dissipated and matter has condensed without increasing the magnetic field by a comparable amount. What are the processes that drive and regulate the rate at which matter reaches this stage? This is a long standing question to which theorists have over the decades offered multiple explanations, focusing on either ambipolar diffusion, turbulence, or magnetic reconnection to decouple matter from the magnetic field and allow the formation of condensations of gas in which stars may form [52].

PRISM observations of the polarization in the far-IR and sub-mm will provide unique clues to understand the role of the magnetic field in star formation. Compared to synchrotron radiation and Faraday rotation, dust polarization images the structure of the magnetic field through an emission process tracing matter. It is best suited to characterize the interplay between turbulence, gravity, and the Galactic magnetic field. PRISM will provide unique data to study magneto-hydrodynamical turbulence because it will drastically increase the spectral range of accurately probed magneto-hydrodynamical modes. The data will provide unprecedented statistical information to characterize the energy injection and energy transfer down to the dissipation scales.

Polarization data from the PRISM survey will have the sensitivity and angular resolution required to map continuously the Galactic magnetic field over the whole sky down to sub-arcminute resolution even at the Galactic poles. The wide frequency range of the mission will make it possible to measure polarization for separate emission components (with distinct temperatures along the line of sight). PRISM will provide a new perspective on the structure of the magnetic field in molecular clouds, independent of grain alignment, by imaging the polarization of CO emission in multiple rotational lines [80]. No project offers 
comparable capabilities. Planck has provided the first all-sky maps of dust polarization with 5 ' resolution but the data is sensitivity limited even at the highest Planck frequency (353 $\mathrm{GHz}$ ). Ground based telescopes at sub-mm and millimeter wavelengths of bright compact sources at arcsecond resolution (for example with ALMA) complement the full-sky survey of extended emission from the diffuse interstellar medium and molecular clouds that only PRISM can carry out.

\subsection{Nature of interstellar dust}

The combination of spectral and spatial information provided by PRISM will provide new tools for studying the interstellar dust, in particular its nature and its evolution. Dust properties (e.g., size, temperature, emissivity) are found to vary from one line of sight to another within the diffuse interstellar medium and molecular clouds. These observations indicate that dust grains evolve in a manner depending on their environment within the interstellar medium. They can grow through the formation of refractory or ice mantles, or by coagulation into aggregates in dense and quiescent regions. They can also be destroyed by fragmentation and erosion of their mantles under more violent conditions. The composition of interstellar dust reflects the action of interstellar processes, which contribute to breaking and reconstituting grains over timescales much shorter than the timescale of injection by stellar ejecta. While there is broad consensus on this view of interstellar dust, the processes that drive its evolution in space are poorly understood [65]. Understanding interstellar dust evolution is a major challenge in astrophysics underlying key physical and chemical processes in interstellar space. In particular, to fully exploit the PRISM data we will need to characterize where in the interstellar medium grains are aligned with respect to the Galactic magnetic field and with what efficiency.

Large dust grains (size $>10 \mathrm{~nm}$ ) dominate the dust mass. Within the diffuse interstellar medium, these grains are cold $(\sim 10-20 \mathrm{~K})$ and emit within the PRISM frequency range. Dipole emission from small rapidly spinning dust particles constitutes an additional emission component, known as anomalous microwave emission. Magnetic dipole radiation from thermal fluctuations in magnetic nano-particles may also be a significant emission component over the frequency range relevant to CMB studies [66]. To achieve the PRISM objectives on CMB polarization, it is necessary to characterize the spectral dependence of the polarized signal from each of these dust components with high accuracy across the sky. This is a challenge but also a unique opportunity for dust studies. The spectral energy distribution of dust emission and the polarization signal can be cross-correlated with the spectral diagnostics of the interstellar medium structure to characterize the physical processes that determine the composition and evolution of interstellar dust. The same data analysis will also elucidate the physics of grain alignment.

\section{Zodiacal light emission}

Zodiacal light emission (ZLE), or thermal emission from dust particles in our Solar System heated by the Sun, is brightest in the mid-infrared [122]. ZLE, however, has been detected even at millimeter wavelengths [158] and constitutes an obstacle to the precise measurement of the total emission and large-scale variations of other emission components. The combination of very sensitive multi-frequency observations with the PRISM imager over a frequency range in which ZLE and galactic dust have significantly different emission spectra and of simultaneous absolute sky intensity at about $1.4^{\circ}$ angular resolution with the PRISM 
spectrophotometer offers a unique opportunity not only to separate zodiacal light emission from galactic dust and other sky emission components, but also to decompose it into main components of different nature.

The bulk of the emission comes from the so-called diffuse cloud, but there are also contributions from a number of IRAS-discovered dust bands as well as a circumsolar ring and an earth-trailing feature. Measurements in the infrared and far infrared have begun to give us information on the size distribution of particles in the diffuse cloud and in the dust bands, but the abundance of millimeter-sized particles is not well-constrained in the circumsolar Ring and its associate earth-trailing feature.

In addition, the Kuiper belt may have detectable amounts of dust, and there is interest in finding dust associated with comets, Jupiter's fourth and fifth Lagrange points, and other substructures in our solar system. While larger objects have been detected in these systems, only a sensitive survey such as PRISM can detect the faint, diffuse emission from larger dust grains which might be at these locations. Thus, while mapping the furthest reaches of the cosmos, PRISM could also help us better understand the diffuse matter in our own neighborhood.

While Galactic emission and the CMB anisotropies themselves will always be brighter than zodiacal emission in the wavelength range near $150 \mathrm{GHz}$, the power spectrum of the emission does reach the $(\mu K)^{2}$ level. This is comparable to the difference in the power spectra arising from different values of cosmological parameters detectable by PRISM, particularly at low and intermediate multipoles. As there is enormous interest in CMB anisotropies at the largest angular scales, even these small contaminants can mask or mimic interesting effects. In the past, there have been claims of interplay between the large-scale CMB anisotropies and emissions or other systematics from our solar system - the so-called anomalies. While this connection seems more tenuous in light of the recent Planck data, the PRISM combination of high precision mapping of temperature and polarization anisotropies with an absolute temperature calibration will either definitely end such speculation or converge toward a more interesting outcome.

An interesting option would be to design the PRISM orbit and scanning strategy such that it views the distant celestial sphere through different columns of interplanetary dust, thereby allowing us to best measure the nearby zodiacal signal and separate it from the background sky emission.

\section{Strawman mission concept}

The science program above requires measuring the sky brightness and polarization at high angular resolution and in many frequency bands across a wide spectral range as well as measuring the absolute spectrum of the sky background with moderate angular and spectral resolution. As a baseline, we propose to perform the best possible spectro-polarimetric sky survey in the 30-6000 GHz frequency range, with two instruments optimized for best joint performance sharing a single platform in orbit around the Sun-Earth L2 Lagrange point: (1) a polarimetric imager (PIM) observing with about 30 broad and 300 narrow spectral bands with a diffraction limited angular resolution and a sensitivity limited by the photon noise of the sky emission itself; and (2) an absolute spectro-photometer (ASP) that will measure sky emission spectra with a spectral resolution between $500 \mathrm{MHz}$ and $15 \mathrm{GHz}$ and an angular resolution of about $1.4^{\circ}$. These complementary instruments will simultaneously map the absolute sky intensity and polarization with high sensitivity and with high spectral 


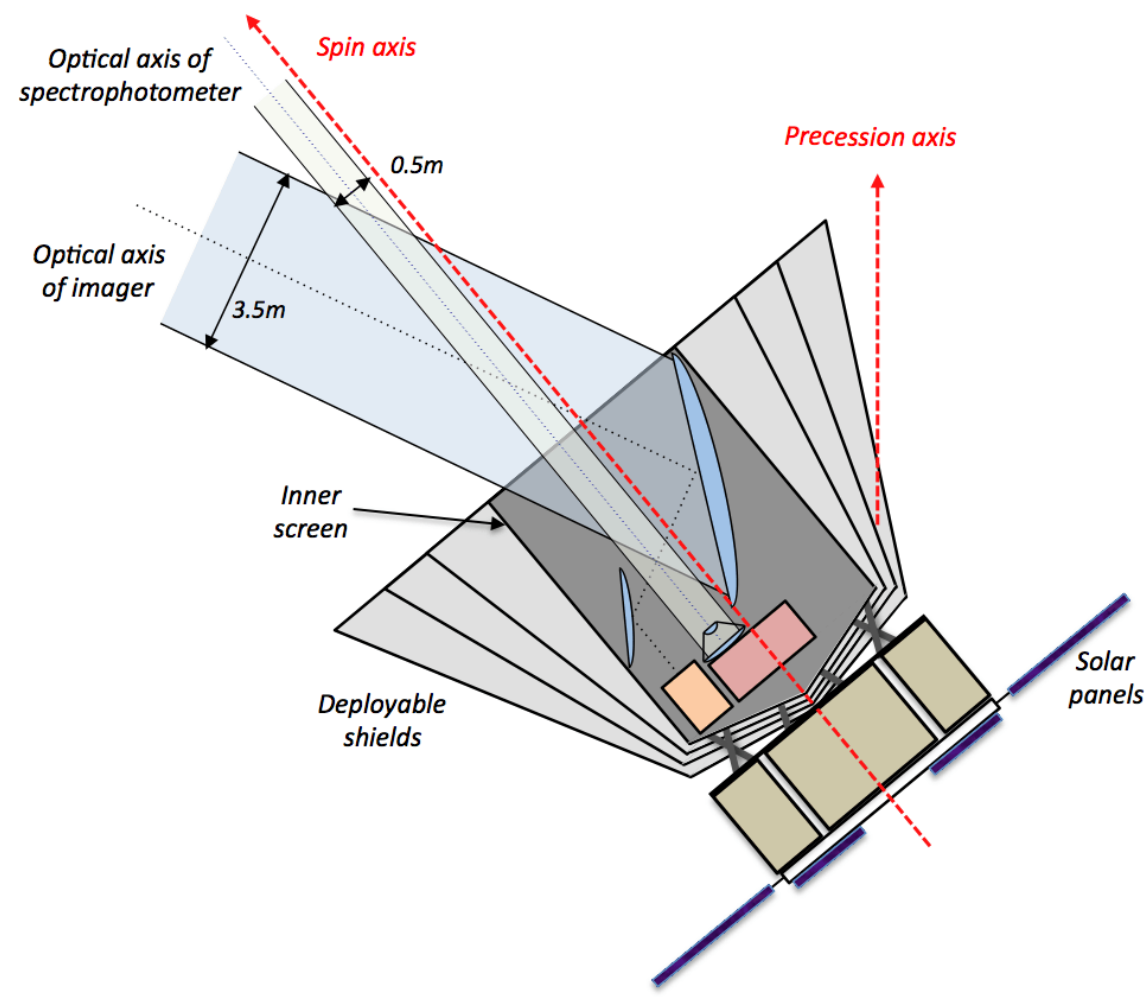

Figure 21. The PRISM spacecraft with its two instruments: (1) the polarimetric imager with a $3.5 \mathrm{~m}$ effective diameter telescope with the field of view at $\approx 30^{\circ}$ with respect to the spacecraft spin axis; (2) the absolute spectrophotometer, with a $1.4^{\circ}$ beam aligned with the spin axis. While the general concept is similar to that illustrated in figure 7 of the white paper proposed to ESA [175], here we propose an option where the spectrophotometer is located between the primary and secondary mirror of the imager, rather than behind the primary, allowing extra space for the optics of the larger telescope of the imager, as well as for the various screens necessary to provide an effective first stage of passive cooling for the telescopes and instruments.

or spatial resolution. The data from both instruments can be binned (in frequency) and smoothed to obtain matching observations with $\delta \nu / \nu \approx 0.25$ and $1.4^{\circ}$ resolution, allowing on-sky inter-calibration on large scales (and hence absolute calibration of the PIM). This will also enable correction of the ASP spectra from foreground contamination using high resolution component maps extracted from PIM data (e.g., $y$-distortion from clusters and galactic emission from regions unresolved in the coarse resolution ASP maps).

Since the scientific outcome of this mission depends on the complementarity of both instruments and on the control of systematic errors, a careful optimization of the ASP and the PIM (number and bandwidth of spectral bands vs. sensitivity) and of the mission (scanning strategy, joint analysis tools) with comprehensive simulations is an essential future phase of the mission study.

\subsection{Instruments}

\subsubsection{The polarimetric imager}

The optical configuration of the polarimetric imager relies on a dual off-axis mirror telescope with a $3.5 \mathrm{~m}$ projected aperture primary mirror (corresponding to a physical size of $3.5 \times$ 


\begin{tabular}{|c|c|c|c|c|c|c|c|c|c|}
\hline \multirow{2}{*}{$\begin{array}{c}\nu_{0} \\
\mathrm{GHz}\end{array}$} & \multirow{2}{*}{$\begin{array}{c}\text { Range } \\
\mathrm{GHz}\end{array}$} & \multirow[t]{2}{*}{$\Delta \nu / \nu$} & \multirow[t]{2}{*}{$n_{\text {det }}$} & \multirow[t]{2}{*}{$\theta_{\text {fwhm }}$} & \multicolumn{2}{|c|}{$\begin{array}{r}\sigma_{I} \text { per det } \\
1 \text { arcmin }\end{array}$} & \multicolumn{2}{|c|}{$\begin{array}{c}\sigma_{(Q, U)} \text { per det } \\
1 \text { arcmin }\end{array}$} & \multirow[t]{2}{*}{ Main molec. \& atomic lines } \\
\hline & & & & & $\mu \mathrm{K}_{\mathrm{RJ}}$ & $\mu \mathrm{K}_{\mathrm{CMB}}$ & $\mu \mathrm{K}_{\mathrm{RJ}}$ & $\mu \mathrm{K}_{\mathrm{CMB}}$ & \\
\hline 30 & $26-34$ & .25 & 50 & $17^{\prime}$ & 61.9 & 63.4 & 87.6 & 89.7 & \multirow{10}{*}{$\begin{array}{r}\mathrm{HCN} \& \mathrm{HCO}^{+} \text {at } 89 \mathrm{GHz} \\
\mathrm{CO} \text { at } 110-115 \mathrm{GHz}\end{array}$} \\
\hline 36 & $31-41$ & .25 & 100 & $14^{\prime}$ & 57.8 & 59.7 & 81.7 & 84.5 & \\
\hline 43 & $38-48$ & .25 & 100 & 12 & 53.9 & 56.5 & 76.2 & 79.9 & \\
\hline 51 & $45-59$ & .25 & 150 & $10^{\prime}$ & 50.2 & 53.7 & 71.0 & 75.9 & \\
\hline 62 & $54-70$ & .25 & 150 & $8.2^{\prime}$ & 46.1 & 50.8 & 65.2 & 71.9 & \\
\hline 75 & $65-85$ & .25 & 150 & $6.8^{\prime}$ & 42.0 & 48.5 & 59.4 & 68.6 & \\
\hline 90 & $78-100$ & .25 & 200 & $5.7^{\prime}$ & 38.0 & 46.7 & 53.8 & 66.0 & \\
\hline 105 & $95-120$ & .25 & 250 & $4.8^{\prime}$ & 34.5 & 45.6 & 48.8 & 64.4 & \\
\hline 135 & $120-150$ & .25 & 300 & $3.8^{\prime}$ & 28.6 & 44.9 & 40.4 & 63.4 & \\
\hline 160 & $135-175$ & .25 & 350 & $3.2^{\prime}$ & 24.4 & 45.5 & 34.5 & 64.3 & \\
\hline 185 & $165-210$ & .25 & 350 & $2.8^{\prime}$ & 20.8 & 47.1 & 29.4 & 66.6 & \multirow{2}{*}{$\mathrm{HCN} \& \mathrm{HCO}^{+}$at $177 \mathrm{GHz}$} \\
\hline 200 & $180-220$ & .20 & 350 & $2.5^{\prime}$ & 18.9 & 48.5 & 26.7 & 68.6 & \\
\hline 220 & $195-250$ & .25 & 350 & $2.3^{\prime}$ & 16.5 & 50.9 & 23.4 & 71.9 & \multirow{8}{*}{$\begin{array}{r}\mathrm{CO} \text { at } 220-230 \mathrm{GHz} \\
\mathrm{HCN} \& \mathrm{HCO}^{+} \text {at } 266 \mathrm{GHz} \\
\mathrm{CO}, \mathrm{HCN} \& \mathrm{HCO}^{+} \\
\mathrm{CO}, \mathrm{HCN}_{2} \mathrm{HCO}^{+} \\
\mathrm{CO}, \mathrm{HCN}^{+} \mathrm{HCO}^{+}\end{array}$} \\
\hline 265 & $235-300$ & .25 & 350 & $1.9^{\prime}$ & 12.2 & 58.5 & 17.3 & 82.8 & \\
\hline 300 & $270-330$ & .20 & 350 & $1.7^{\prime}$ & 9.6 & 67.1 & 13.6 & 94.9 & \\
\hline 320 & $280-360$ & .25 & 350 & $1.6^{\prime}$ & 8.4 & 73.2 & 11.8 & 103 & \\
\hline 395 & $360-435$ & .20 & 350 & $1.3^{\prime}$ & 4.9 & 107 & 7.0 & 151 & \\
\hline 460 & $405-520$ & .25 & 350 & $1.1^{\prime}$ & 3.1 & 156 & 4.4 & 221 & \\
\hline 555 & $485-625$ & .25 & 300 & $55 "$ & 1.6 & 297 & 2.3 & 420 & \\
\hline \multirow[t]{2}{*}{660} & $580-750$ & .25 & 300 & $46 "$ & 0.85 & 700 & 1.2 & 990 & \\
\hline & & & & & $\mathrm{nK}_{\mathrm{RJ}}$ & $\mathrm{kJy} / \mathrm{sr}$ & $\mathrm{nK}_{R J}$ & $\mathrm{kJy} / \mathrm{sr}$ & \\
\hline 800 & $700-900$ & .25 & 200 & $38 "$ & 483 & 9.5 & 683 & 13.4 & \multirow{5}{*}{$\mathrm{N}-\mathrm{II}$ at $1461 \mathrm{GHz}$} \\
\hline 960 & $840-1080$ & .25 & 200 & $32 "$ & 390 & 11.0 & 552 & 15.6 & \\
\hline 1150 & $1000-1300$ & .25 & 200 & $27 "$ & 361 & 14.6 & 510 & 20.7 & \\
\hline 1380 & $1200-1550$ & .25 & 200 & $22 "$ & 331 & 19.4 & 468 & 27.4 & \\
\hline 1660 & $1470-1860$ & .25 & 200 & $18 "$ & 290 & 24.5 & 410 & 34.7 & \\
\hline 1990 & $1740-2240$ & .25 & 200 & $15 "$ & 241 & 29.3 & 341 & 41.5 & \multirow{5}{*}{ O-III at $3393 \mathrm{GHz}$} \\
\hline 2400 & $2100-2700$ & .25 & 200 & $13 "$ & 188 & 33.3 & 266 & 47.1 & \\
\hline 2850 & $2500-3200$ & .25 & 200 & $11 "$ & 146 & 36.4 & 206 & 51.4 & \\
\hline 3450 & $3000-3900$ & .25 & 200 & $8.8 "$ & 113 & 41.4 & 160 & 58.5 & \\
\hline 4100 & $3600-4600$ & .25 & 200 & $7.4 "$ & 98 & 50.8 & 139 & 71.8 & \\
\hline 5000 & $4350-5550$ & .25 & 200 & $6.1 ”$ & 91 & 70.1 & 129 & 99.1 & O-I at $4765 \mathrm{GHz}$ \\
\hline 6000 & $5200-6800$ & .25 & 200 & $5.1 "$ & 87 & 96.7 & 124 & 136 & O-III at $5786 \mathrm{GHz}$ \\
\hline
\end{tabular}

Table 1. The 32 broad-band channels of the polarized imager with a total of 7600 detectors. Sensitivities are averages for sky regions at galactic latitude and ecliptic latitude both higher than $30^{\circ}$. The mission sensitivity per frequency channel is the sensitivity per detector divided by $\sqrt{n_{\text {det }}}$. The angular resolution $\theta_{\text {fwhm }}$ conservatively assumes under-illumination of the primary mirror by a Gaussian with $30 \mathrm{~dB}$ edge taper to reduce sidelobe pickup.

$4.2 \mathrm{~m}$ ) and a $0.8 \mathrm{~m}$ diameter secondary mirror, coupled to a multi-band polarimeter. The broad-band PIM comprises 32 main channels of $\delta \nu / \nu \approx .25$ with dual-polarized pixel arrays (table 1). An instrinsic detector noise equal to the photon noise from the sky signal is assumed as well as a conservative optical efficieny of $40 \%$. At frequencies below $700 \mathrm{GHz}$, the emphasis is on the sensitivity and control of systematics for CMB and SZ science. Most of the frequency range will also be covered at higher spectral resolution $(\delta \nu / \nu \approx .025$ or better) to map spectral lines. The exact frequency channels are still to be optimized and are not listed in table 1.

A simple calculation assuming a focal plane unit based on classic feed horn coupled detectors for each spectral band, leads to a focal plane diameter of about $80 \mathrm{~cm}$. While this concept is well understood, new technologies will help to reduce the focal plane size and the number of pixels (see discussion on detectors below). 


\begin{tabular}{|c|c|c|c|c|c|}
\hline $\begin{array}{c}\text { Band } \\
(\mathrm{GHz})\end{array}$ & $\begin{array}{c}\text { Resolution } \\
(\mathrm{GHz})\end{array}$ & $\begin{array}{c}A \Omega \\
\left(\mathrm{cm}^{2} \mathrm{sr}\right)\end{array}$ & $\begin{array}{c}\text { Background } \\
(\mathrm{pW})\end{array}$ & $\begin{array}{c}\mathrm{NEP} \nu \\
\left(\mathrm{W} / \mathrm{m}^{2} / \mathrm{sr} / \mathrm{Hz} \times \sqrt{\mathrm{s}}\right)\end{array}$ & $\begin{array}{c}\text { Global 4-yr mission } \\
\text { sensitivity }\left(\mathrm{W} / \mathrm{m}^{2} / \mathrm{sr} / \mathrm{Hz}\right)\end{array}$ \\
\hline \hline $30-6000$ & 15 & 1 & 150 & $1.8 \times 10^{-22}$ & $1.8 \times 10^{-26}$ \\
\hline $30-500$ & 15 & 1 & 97 & $7.0 \times 10^{-23}$ & $7.2 \times 10^{-27}$ \\
$500-6000$ & 15 & 1 & 70 & $1.7 \times 10^{-22}$ & $1.7 \times 10^{-26}$ \\
\hline $30-180$ & 15 & 1 & 42 & $3.5 \times 10^{-23}$ & $3.6 \times 10^{-27}$ \\
$180-600$ & 15 & 1 & 57 & $6.3 \times 10^{-23}$ & $6.5 \times 10^{-27}$ \\
$600-3000$ & 15 & 1 & 20 & $7.4 \times 10^{-23}$ & $7.6 \times 10^{-27}$ \\
$3000-6000$ & 15 & 1 & 28 & $1.6 \times 10^{-22}$ & $1.6 \times 10^{-26}$ \\
\hline
\end{tabular}

Table 2. FTS performance of three possible configurations for photon noise limited detectors, operating in a radiative background comprising $\mathrm{CMB}$, high galactic latitude interstellar dust, CIB, and high ecliptic latitude interplanetary dust emission. With an entrance pupil $50 \mathrm{~cm}$ in diameter, the baseline throughput is $\sim 1 \mathrm{~cm}^{2} \mathrm{sr}$ and the angular resolution $1.4^{\circ}$. The theoretical monopole sensitivity for each spectral bin is reported in the last column assuming 4 years of observation and $75 \%$ useful sky. The actual sensitivity taking into account efficiency factors can be $2-3$ times worse. Line 1 is a configuration with an ultra-wide spectral coverage obtained with one detector in both output ports. In lines 2-3 the detectors at the output ports are sensitive to different bands. In lines 4-7 each output port is split into two sub-bands using dichroics to minimize photon noise in the low-frequency bins.

\subsubsection{The absolute spectrophotometer}

A Martin-Puplett Fourier Transform Spectrometer (FTS) will allow for a large throughput and deliver high sensitivity, differential measurements (in which the sky is compared to an internal blackbody calibrator as in COBE-FIRAS). This setup also allows for an adjustable spectral resolution that can be changed in the course of the mission. Dichroics at the two output ports can optionally split the full $30-6000 \mathrm{GHz}$ range into sub-bands with reduced photon noise. The instrument is cooled at $2.7 \mathrm{~K}$, so that the bolometric detector sensitivity is limited by photon noise from the sky. Two operating modes are available: high-resolution $(\Delta \nu \sim 0.5 \mathrm{GHz})$ and low-resolution $(\Delta \nu \sim 15 \mathrm{GHz})$. The sensitivity of the high-resolution mode is 30 times worse than for the low-resolution mode. The instrument beam is aligned with the spin axis of the satellite, so that precession has a negligible effect during the interferogram scan $(\sim 1 \mathrm{~s} / 10 \mathrm{~s}$ long in the low-res/high-res mode). The main characteristics for three possible configurations of the instrument are detailed in table 2.

Using detectors with $A \Omega \sim 1 \mathrm{~cm}^{2}$ sr and angular resolution $\sim 1.4^{\circ}$, we estimate that the CIB can be measured with $S / N=10$ in a fraction of a second at $1500 \mathrm{GHz}$ and in $\sim 10$ seconds at $140 \mathrm{GHz}$, while a $y$-distortion $\sim 10^{-8}$ can be measured with $S / N=10$ at $350 \mathrm{GHz}$ in two hours of integration. Recombination lines could be measured integrating over the whole mission if the overall stability of the instrument and the quality of the reference blackbody are sufficient.

The main issue for this instrument is the control of systematic effects. The instrument design allows for a number of zero tests and cross-checks on the data. The main problem is to control the blackness of the reference and calibration blackbodies with the required accuracy. Reflectivities lower than $R=-50 /-60 \mathrm{~dB}$ have been obtained in the frequency range of interest in the Planck and ARCADE references. Building on these experiences, we plan to achieve $R<-70 \mathrm{~dB}$ through a combination of electromagnetic simulations and laboratory emissivity measurements on improved shapes and space qualified materials. 


\subsubsection{Synergy of the two instruments}

There is significant added value to observing with the two instruments together on the same payload. The observed data cubes from the absolute spectrophotometer comprise CMB and foreground emission, mixed and blended by both superposition along the line of sight and averaging across several lines of sights smeared by the $1.4^{\circ}$ beam. Foreground emissions include galactic dust cirrus, spectral lines in molecular clouds, extragalactic sources, clusters of galaxies, and variable radio sources.

To achieve the ambitious precision goals of the spectrophotometer for CMB spectral distortion measurements, it is essential that these sources of foreground emission are characterized and subtracted using the narrow beam imager data. Without the high angular resolution data from the imager, precise measurement of the CMB spectral distortions are possible only on a reduced fraction of clean sky, resulting in significant degradation of its global sensitivity. The interpretation of the observations would also rely on how some of the components are modelled.

Conversely, absolute spectrometer data are essential to measure the zero-level of the imager maps (and hence to measure absolute column densities for ISM emissions, the total emission of the CIB, etc.) as well as for accurate absolute photometric calibration of the imager channels across frequencies (with a goal of $0.05 \%$ ). In this way the absolute calibration is transferred to the imaging polarimeter to yield high-resolution absolute maps of sky emission. The precise calibration of all measurements across frequencies is key to component separation, in particular to extracting with minimal contamination faint sources of radiation identified using the frequency dependence of their emission.

\subsection{Experimental challenges}

The following subsections address some of the technologically challenging requirements of the PRISM mission. The main features essential to carry out the proposed science program are the large telescope, necessary for the high angular resolution needed for SZ, extragalactic, and galactic science; telescope cooling to temperature below $10 \mathrm{~K}$, essential for the sensitivity at frequencies above $1 \mathrm{THz}$; a large focal plane of thousands of detectors, for sensitivity and spectral coverage; the quality of the calibration, essential for accurate component separation; and the combination of the co-observing imager and spectrophotometer, to measure both the absolute level of all maps and absolute spectrum of CMB emission, as well as the sky brightness and polarization fluctuations down to the smallest possible angular scales.

\subsubsection{Telescope}

The telescope is designed with the largest possible monolithic primary mirror to obtain the best angular resolution achievable within the budget of a large ESA mission. The surface accuracy must allow for observations down to a wavelength of 50 microns. An off-axis configuration is preferred because it allows for a larger focal plane area and better polarization properties (without, in particular, struts holding the secondary mirror in the optical path).

The science achievable with PRISM depends on the angular resolution, especially for detecting galaxy clusters and measuring their peculiar velocities, and for resolving the distant galaxies that constitute the CIB. The dependance of mission performance on telescope size, however, is not very steep, so that the appropriate compromise between feasibility, cost, and scientific requirements can be made during the mission design phase.

Figure 22 shows the envisaged PRISM layout for both instruments. The primary mirror of the imager is $3.5 \mathrm{~m} \times 4.2 \mathrm{~m}$ in size. The design and production of the large telescope will 

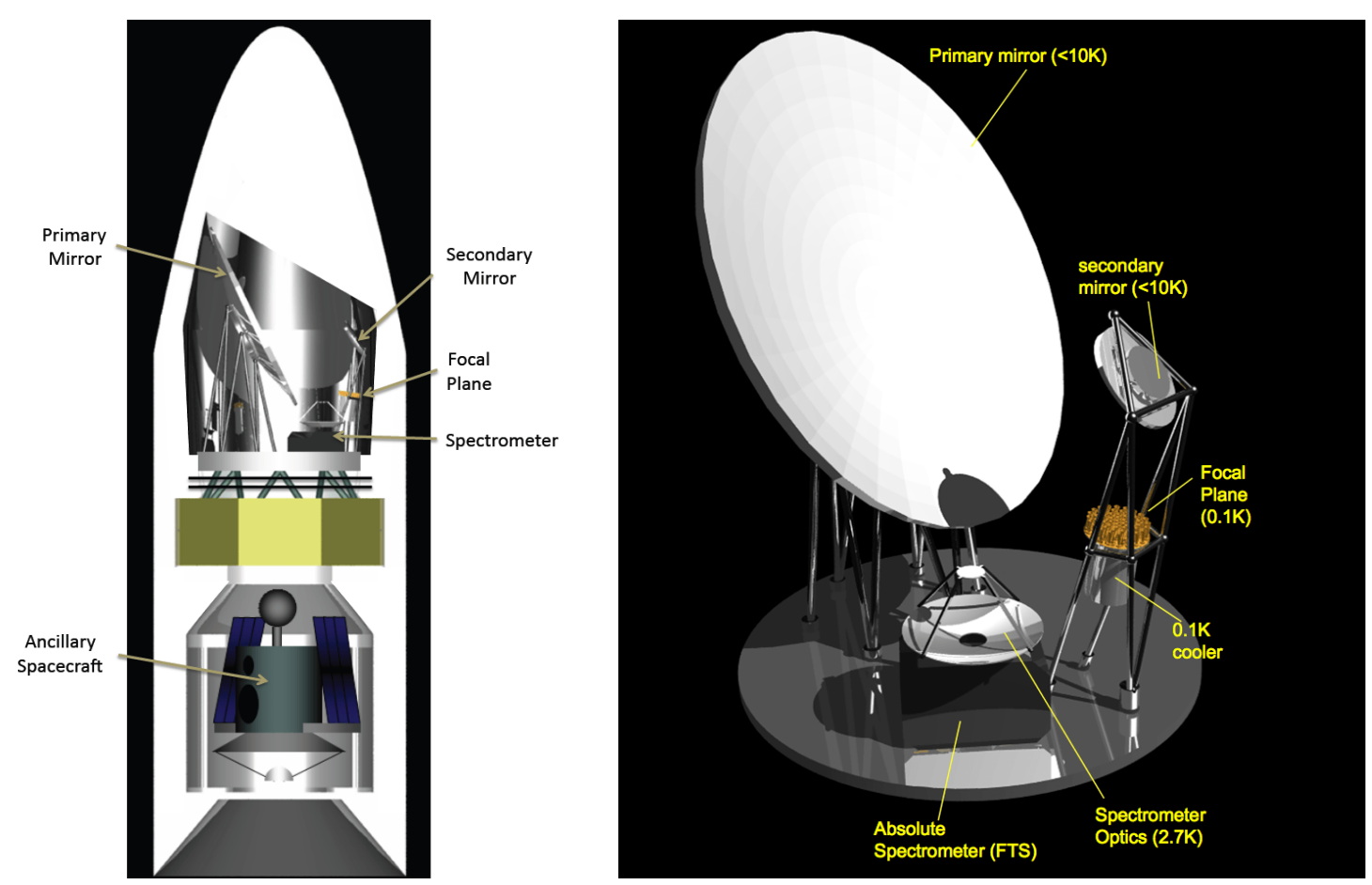

Figure 22. Left panel: the two satellites inside the Ariane-V fairing. The main satellite is on top (the Sun shields, in stowed position, are not shown). The bottom satellite (displayed in the Ariane- $\mathrm{V}$ SYLDA for a possible launch configuration) provides a set of calibrators for the observatory and the high gain, high data rate communication system. Right panel: detail of main satellite, showing a possible layout for the two PRISM instruments with the locations of the off-axis telescope with a 3.5 $\mathrm{x} 4.2 \mathrm{~m}$ primary, the polarimeter focal plane, and the spectrometer.

benefit from the Herschel mission heritage ( $3 \mathrm{~m}$ class telescope, with instruments operated down to 60 microns), and the studies and development activities for the SPICA mission $(\sim 3 \mathrm{~m}$ diameter primary mirror cooled to about $5 \mathrm{~K}$ and instruments operating down to 5 microns).

Actively cooling the telescope to $4 \mathrm{~K}$ (mission objective) instead of $40 \mathrm{~K}$ (achievable by passive cooling) substantially improves the sensitivity, especially for frequencies above $200 \mathrm{GHz}$ (figure 23). The goal is to reach a mirror temperature of 4 to $5 \mathrm{~K}$ with a requirement of $10 \mathrm{~K}$, necessary to map the complete sky at frequencies up to $6 \mathrm{THz}$ (50 microns) with a sensitivity better than that of Herschel SPIRE in large survey observations (which cover approximately $10 \%$ of the sky).

Figure 23 shows the sensitivity of a single PRISM detector as a function of telescope temperature, assuming detector noise equal to photon noise, and $25 \%$ bandwidth. At the highest frequencies (above $3 \mathrm{THz}$, i.e., below 100 microns), where zodiacal light emission dominates the sky contribution to the photon noise, a temperature of $10 \mathrm{~K}$ or lower is sufficient to reach the best possible detector sensitivity with a sky-dominated background. Below $200 \mathrm{GHz}$ little is gained by actively cooling the telescope, as compared to passive cooling to $30-40 \mathrm{~K}$. The intermediate frequencies (in particular the 400-2000 GHz range) are where the best sensitivity is achieved with the coldest possible mirror temperature. While increased sensitivity is not critical for the detection of dusty galaxies (which is background limited), it is important for the detection of galaxy clusters via the thermal SZ effect, which is one of the main mission objectives. 


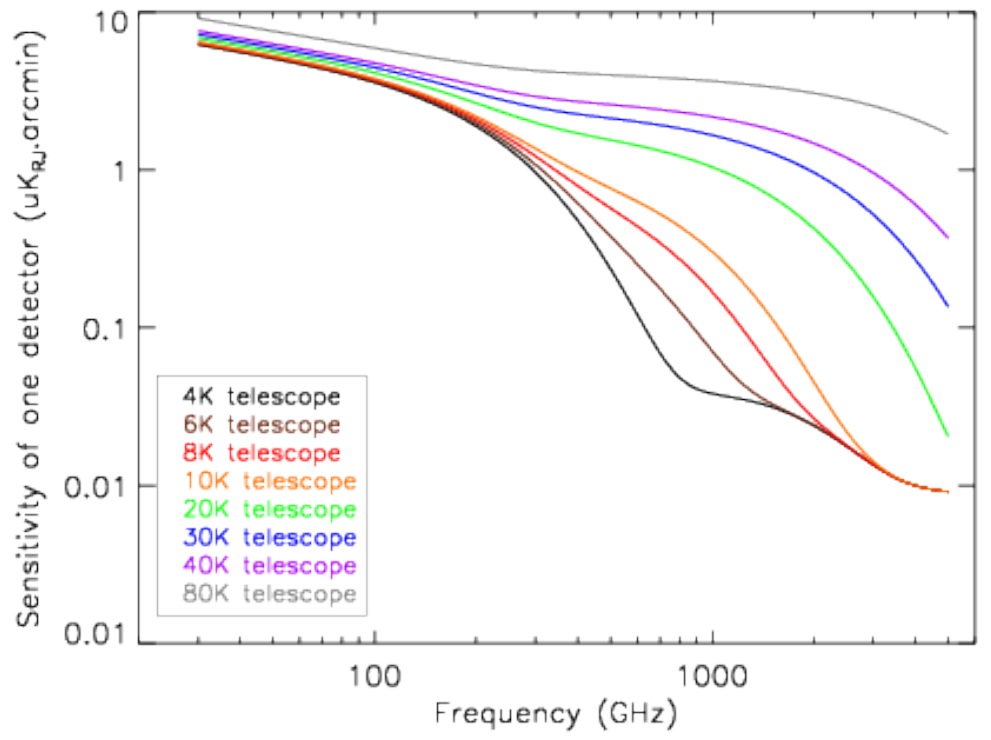

Figure 23. Sensitivity of one PRISM detector as a function of frequency for various temperatures of a $2 \%$ emissive telescope. The sensitivity is plotted as the average of sky regions at galactic and ecliptic latitudes higher than $30^{\circ}$. The background comprises CMB, thermal dust emission, CIB, zodiacal light, and telescope emission (assuming $2 \%$ total telescope emissivity).

\subsubsection{Cooling chain}

In addition to the cooling of the large telescope of the polarimetric imager to below $10 \mathrm{~K}$, the $50 \mathrm{~cm}$ telescope of the spectrophotometer will be cooled to $2.7 \mathrm{~K}$, and the detectors will operate at sub-Kelvin temperatures.

The cooling chain will rely on a first stage of passive cooling of the payload using a set of deployable V-grooves, as well as an inner solid cylindrical shield (figure 21). The large mirror of the polarimetric imager will be cooled using a cryogenic chain that will use as a starting point the study of the SPICA 3.2 meter telescope cooling system, for which a temperature of 5-6 K can be achieved. PRISM has a mirror about $30 \%$ bigger in area, but the requirement on the telescope temperature $(<10 \mathrm{~K})$ is less stringent (however with an objective of $4 \mathrm{~K}$ if possible for optimal sensitivity). The focal planes of both instruments will be cooled to $0.1 \mathrm{~K}$ using a cryogenic system adapted from the dilution refrigerator onboard Planck but with continuous helium recycling for a longer mission duration of 4 years (baseline) or beyond.

\subsubsection{Scan strategy}

The observing strategy must provide: (1) full sky coverage for both instruments; (2) crosslinked scan paths and observation of all sky pixels in many orientations for all detectors of the polarimetric imager; (3) fast scanning of the imager to avoid low-frequency drifts; (4) slow scanning for the spectrophotometer field of view to allow for few seconds long interferogram scans with negligible depointing; (5) avoiding direct solar radiation on the payload.

These requirements can be satisfied by a spinning spacecraft with the beam of the spectrophotometer aligned along the spin axis and the imager field of view offset by $\theta_{\text {spin }} \approx 30^{\circ}$ (figure 21). During each spacecraft rotation (with $\omega_{\text {spin }}$ of a few rpm), the field of view of the imager scans circles of diameter $\approx 2 \theta_{\text {spin }}$ while the beam of the spectrophotometer rotates in 
place. A slow precession of the spin axis (with a period between a few hours and one day) with a precession angle $\theta_{\text {prec }} \approx 45^{\circ}$ results in slow scans of the spectrophotometer on large circles of diameter $2 \theta_{\mathrm{prec}}$. Finally, the precession axis evolves by about $1^{\circ}$ per day along the ecliptic plane to keep the payload away from the Sun, and also slowly moves perpendicular to the ecliptic plane so as to map the ecliptic poles. Deployable screens isolate the payload from the heat from the Sun, providing a first stage of passive cooling to $\approx 40 \mathrm{~K}$.

\subsubsection{Polarization modulation}

The precise measurement of polarization imposes stringent requirements on the instrument characterization. Polarization modulation provides essential redundancy and robustness tests. The polarization modulation baseline for PRISM, similar to the solution proposed in the previous SAMPAN and EPIC studies, relies on the scanning strategy and the rotation of the entire payload. However alternative strategies such as the use of a half-wave plate in front of the focal plane (the receivers being the major source of instrumental polarization) will be investigated during a trade-off analysis.

\subsubsection{Detectors}

The PRISM mission will observe the sky using a focal plane of 7600 broadband detectors in the millimeter to far infrared frequency range. It is also planned to include a number of narrow band detectors for line monitoring and limited spectroscopy.

Direct detectors (such as TES bolometers, CEBs or KIDs) are the most sensitive detectors at millimeter wavelengths. Bolometers have achieved in-flight performance with the Planck [206] and Herschel [82] missions close to the instrinsic photon noise limit. Large bolometer arrays with thousands of pixels are currently being used on large ground-based telescopes.

These technologies have not yet been demonstrated to be viable for 30 to $70 \mathrm{GHz}$. Nevertheless, it is likely that their efficiency at low frequencies will improve over the next few years. For instance, the studies in ref. [116] have shown that $70 \mathrm{GHz} \mathrm{CEBs}$ could lead to NEPs of (few) $\times 10^{-18} \mathrm{~W} \cdot \mathrm{Hz}^{1 / 2}$. As an alternative solution, the PRISM instruments could take advantage of the recent breakthroughs in cryogenic HEMT technology, with sensitivities predicted to reach 2-3 times the quantum limit up to 150-200 GHz (instead of 4-5 times up to $100 \mathrm{GHz}$ so far). The fact that these devices allow for cryogenically cooled miniaturized polarimeter designs will simplify the thermo-mechanical design. Hence, while a single detector technology throughout the instruments would be preferable, the option of using a combination of HEMTs and bolometers remains open (tables 3 and 4). While the whole focal plane could be built from pixels based only on direct detectors, the detector technology and optical coupling concept will probably vary across the spectral domain from 100 to $6000 \mathrm{GHz}$. Table 3 lists potential technology candidates.

In order to reduce the size of the focal plane, multichroic pixels could be used where one antenna can feed 2 or 3 spectral bands ( 4 or 6 detectors). For instance, waveguide filtering, which could be adapted to split the bands, has been improved lately [120], and multichroic TES detectors are being developed and tested for PolarBear II and LiteBird. Moreover a recent ESA ITT has been released in the last few months in order to study large focal plane concepts in Europe. 


\begin{tabular}{|c|c|c|c|}
\hline Frequency $(\mathrm{GHz})$ & Technology & TRL & Used for \\
\hline $30-90$ & HEMT & 9 & WMAP, Planck-LFI \\
& Bolometers & $2-3$ & \\
\hline $90-400$ & Feedhorn coupled TES & 7 & Ground/balloon projects, SPICA \\
& Antenna coupled TES & 5 & PolarBear, SPIDER \\
& KIDs & 5 & NIKA, MUSE \\
& CEBs & 4 & Research \\
\hline $400-2000$ & Antenna coupled TES & 5 & PolarBear, SPIDER \\
& Planar Resistive Bolometers & 9 & Herschel-PACS \\
\hline $2000-6000$ & Planar Resistive Bolometers & 9 & Herschel-PACS \\
& HEB, SIS & 9 & Herschel-HIFI \\
\hline
\end{tabular}

Table 3. Available detector technologies in the PRISM frequency range with Technology Readiness Level (TRL) and experiments that have observed with the corresponding detector technology.

\begin{tabular}{|c|c|c|c|c|c|c|}
\hline$\nu_{c}$ range & Req. NEP & Req. $\tau$ & \multicolumn{3}{|c|}{ Focal plane technology } \\
\hline \multirow{2}{*}[\mathrm{GHz}]{} & {$\left[10^{-18} \mathrm{~W} / \sqrt{\mathrm{Hz}}\right]$} & \multirow{2}{*}[ms]{} & Detector technology & \multicolumn{2}{c|}{ Optical coupling } \\
\cline { 4 - 7 } & & Baseline & Backup & Baseline & Backup \\
\hline \hline $30-75$ & $3.3-5.7$ & $2.96-1.18$ & TES & HEMT & MPA/CSA & HA \\
\hline $90-320$ & $4.6-7$ & $1.18-0.4$ & TES & KIDS & HA+POMT & MPA \\
\hline $395-660$ & $0.94-3.1$ & $0.4-0.13$ & TES & KIDS & MPA/CSA & LHA \\
\hline $800-6000$ & $0.011-0.63$ & $0.13-0.01$ & KIDS & HEB/CEB & MPA/CSA & LHA \\
\hline
\end{tabular}

Table 4. Required NEP (Noise Effective Power) and time constants for various frequency ranges and corresponding baseline and backup focal plane technology. TES: Transition Edge Sensors (Technology Readiness Level 5); HEMT: High Electron Mobility Transistor (TRL 5); KID: Kinetic Inductance Detector (TRL 5); HEB: Hot Electron Bolometer (TRL 4); CEB: Cold Electron Bolometer (TRL 3); HA: Horn Array (TRL 9); LHA: Lithographed Horn Array (TRL 5); MPA: Multichroic Planar Antenna (TRL 4); CSA: Crossed Slot Antenna (TRL 5); POMT: Planar Ortho-Mode Transducer (TRL 5).

\subsubsection{Detector time constants}

The rapid scanning by PRISM requires fast detector time constants, of order $1 \mathrm{~ms}$ at 100 $\mathrm{GHz}$, down to $\sim 10 \mu \mathrm{s}$ at $6 \mathrm{THz}$. These time constants are challenging (especially at high frequencies), but have already been achieved using recent TESs, KIDs and CEBs.

\subsection{Ancillary spacecraft}

We propose that the mission include a small ancillary spacecraft serving the following functions:

Telecommunication: the high resolution mapping of the full sky with the many detectors of PRISM with a lossless compression of 4 gives a total data rate of $\sim 350 \mathrm{Mbit} / \mathrm{s}$ (out of which $300 \mathrm{Mbit} / \mathrm{s}$ is from the channels above $700 \mathrm{GHz}$ ). Further on-board reduction by a factor $\sim 10-20$ can be achieved by averaging the timelines of detectors following each other on the same scan path (after automatic removal of spikes due to cosmic rays) to yield a total data rate $<40 \mathrm{Mbit} / \mathrm{s}$ (a few times higher than Euclid or Gaia). While a phased-array antenna or counter-rotating antenna on the main spacecraft is a possible 
solution, decoupling the communication function from the main spacecraft using an ancillary spacecraft as an intermediate station for data transmission has several advantages. Most notably, telecommunications with Earth use power from the ancillary spacecraft, allowing power from the main spacecraft to be redeployed for payload cooling. Using a large pointed antenna and the additional power available for telemetry increases the data rate to Earth. Finally, because the main spacecraft does not have to be pointed toward Earth, a more flexible scanning strategy is possible allowing better polarization modulation and optimal distribution of the observation time over the sky.

In-flight calibration: the formidable PRISM design challenge is to ensure that performance is limited by detector noise rather than by systematic effects and calibration uncertainties. While pre-flight calibration is necessary, an ancillary spacecraft fitted with calibrated, polarized sources could be used for precise in-flight calibration of the polarization response and polarization angles of the detectors as well as for main beam and far sidelobe measurements down to extremely low levels (below $-140 \mathrm{~dB}$ ) at several times during the mission lifetime.

While the relative position of the two satellites will have to be monitored precisely to achieve a goal of $0.05 \%$ accuracy in relative calibration, this is well within what can be achieved with standard ranging techniques. No precise formation flying is required.

\section{Competition and complementarity with other observations}

In this last section, we discuss the PRISM mission in the general framework of relevant current, planned, and proposed observations.

\subsection{Ground-based and balloon-borne CMB B-mode experiments}

A key scientific objective of PRISM is the detection and characterization of the CMB polarization B-modes and corresponding constraints of inflationary models (section 5). Efforts from the ground and balloons are also seeking to discover the B-modes despite considerable obstacles due to atmospheric interference and an unstable observing environment. Observations from suborbital platforms to detect CMB B-modes, both from primordial tensor modes and from lensing, are a very active area of current research. Over the last few years nearly two dozen concepts for dedicated experiments have been described in talks and in the literature, with deployments that are ongoing or envisaged for the forthcoming decade. Some of these proposals involve multiple, staged deployments of progressively more advanced and sensitive experimental set-ups. Hence it is likely that the status of our knowledge will evolve significantly before the launch of PRISM.

To date two suborbital experiments QUIET [178] and BICEP [17] have published analyses of their first data sets, setting up upper limits on the B-mode power and $r$ parameter based on the B-mode polarization, $r \lesssim 0.7$ (95\% c.l.). A few more experiments are currently in operation or in the data analysis stage, including BICEP/Keck [18], SPTpol [135], ABS [1], EBEX [70], POLARBEAR [171], and a few more scheduled for the deployment within a year or so (e.g., QUIJOTE [179], ACTpol [143] and SPIDER [76]). These experiments should start producing results within the next few years, and at the end of that period are expected to have sufficient sensitivity to provide a convincing direct first detection of the lensing generated B-mode spectrum and start probing primordial B-modes with $r$ below 0.1 and possibly down to $r \sim 0.05$ (95\% c.l.). A limit on $r$ at about the same level can be also expected 
from Planck in the coming year. Recently, the presence of lensing B-modes has been confirmed by the SPTpol team, with a cross-correlation analysis of CMB polarization with CIB measurements at 500 microns from Herschel SPIRE [85].

On the timescale of a decade or so, more advanced versions of ground-based and balloonborne experiments as well as some new ones, including technologically novel proposals such as the QUBIC bolometric interferometer [177], should reach the sensitivities to detect the gravity waves contribution down to sub $r \sim 0.01$ levels and potentially as low as $r \sim 0.007$ (e.g., POLARBEAR-EXT [171], PIPER [155], LSPE [129]), although this prediction is probably optimistic. The performance of these last experimental set-ups is typically considered near the limit of what can be achieved with suborbital observations because of the considerable limitations due to atmospheric opacity and emission, far-side lobe pickup from the ground, and unstable observing conditions that make controlling systematic errors very difficult (particularly on large angular scales where the B-mode signal is largest). In addition, forecasts of $r$ from ground-based experiments are often impressive but assume very simple foreground contamination modelling. For this reason a tentative detection of $r$ from the ground would in fact provide a strong motivation for a confirmation and more precise characterization from space.

In the light of these forecasts, PRISM would improve by almost two orders of magnitude what can be achieved with the most advanced of the above suborbital experiments owing to PRISM's full sky coverage, outstanding sensitivity, and immunity against systematics inherent to suborbital experiments. PRISM would also carry out precise measurements of the B-mode spectrum extending to the largest angular scales, which are inaccessible to any single suborbital experiment. Moreover, systematic effects will play a major role in determining the actual performance of suborbital observations. In contrast, a space mission such as PRISM will allow exquisite control of systematic effects, thanks to the combination of benefits of the space environment at L2 and its advanced hardware design. The overall gain in performance from such a mission is therefore very likely to exceed the estimates based solely on the statistical considerations quoted earlier.

We note that even if $r$ happens to be large (i.e., $r \gtrsim 0.01$ ) so that first hints of a Bmode signal could potentially be seen by some of the most sensitive suborbital experiments (which is by no means guaranteed), its precise characterization and scientific exploitation is possible only with a satellite mission. If $r$ is small, a very sensitive space mission with precise characterization of the instrument and control of systematics, and enough frequency bands to deal with complex foreground emission, is required to make a detection. PRISM is designed to achieve this objective and give the final word on CMB primordial B-modes. In addition, it will provide the best possible full-sky measurement of lensing B-modes, essential to investigate the distribution of mass in the high redshift universe.

\subsection{Other space CMB projects}

Two US space missions concepts, CMBPol and PIXIE, and one Japanese, LiteBird, have been proposed in the past few years. In Europe, the PRISM proposal follows the previously proposed SAMPAN satellite in France, and the BPol and COrE projects, proposed as Mclass missions to ESA in 2007 and 2010 respectively. None of these missions, however, has been funded or approved yet. Among the current space mission concepts, PRISM is the most ambitious and encompasses the broadest science case, B-mode polarization being only one of several main scientific objectives of the mission. 
LiteBird is a highly-targeted, low-cost Japanese B-mode mission concept, in many respects similar to the BPol mission proposed to ESA in 2007. It is designed to detect B-modes at the level of $r \sim 10^{-3}$, a sensitivity that it should be able to achieve assuming that the foregrounds are not too complicated. However, LiteBird lacks the angular resolution needed to make significant contributions to other key science objectives, and hence is not a competitor for PRISM. If LiteBird is approved, it will probe the B-mode range down to one order of magnitude lower than what can be done from the ground. PRISM will be about 10 times more sensitive yet, and more robust to contamination by complex foreground emission.

The US EPIC-CS mission is the most ambitious of the previously proposed mission concepts. EPIC-CS is in some respects similar to the present proposal, also targeting high resolution $\mathrm{CMB}$ science (in particular $\mathrm{CMB}$ lensing and some galaxy cluster science), but has considerably less frequency coverage, fewer frequency bands, and no absolute spectral capability.

The PIXIE mission concept is an improved version of the FIRAS spectrometer, which aims at performing absolute spectroscopy and measuring large-scale CMB B-modes simultaneously. One of its major limitations is the effective resolution of only $2.6^{\circ}$. The absolute spectrophotometer of PRISM is very similar conceptually to that of PIXIE, but it will benefit from slightly higher angular resolution and better sensitivity, and is not the instrument used to measure polarization with PRISM. It will also, most importantly, benefit from the additional imager observations to clean-up the observed spectra from the contamination due to small scale localized emission (from SZ galaxy clusters, clouds of emission in the ISM, and strong IR and radio point sources).

A small satellite such as LiteBIRD of PIXIE, if funded and launched before PRISM, could make a first detection of CMB B-modes - or not. PRISM will then either provide an accurate measurement, or make the ultimate attempt at detecting primordial CMB polarization B-modes and definitively settle the question.

\subsection{Galaxy clusters observations}

By the time PRISM flies, several large cluster surveys will have been completed, surpassing the first generation of SZ surveys from SPT, ACT and Planck. These new generation surveys, most notably in the X-ray and optical/near IR bands, will complement the PRISM cluster catalog with invaluable additional information. In addition, large-aperture ground-based submillimeter telescopes, such as CCAT, ${ }^{5}$ will offer the exciting possibility of detailed follow-up of PRISM clusters at high angular resolution, enabling important studies of the gas physics of clusters out to high redshifts.

eROSITA is an X-ray instrument onboard the SRG Russian satellite to be launched in 2014. The mission's principal goal is to explore cosmological models using galaxy clusters. Forecasts predict that eROSITA, 20-30 times more sensitive than ROSAT, will detect $\sim 10^{5}$ clusters at more than $100 \mathrm{X}$-ray photon counts, which is sufficient to provide a good detection and in many cases to detect the source as extended in X-rays. The main survey provides a good sample of galaxy clusters typically out to $z=1$ with some very massive and exceptional clusters at larger distance.

The large majority of these clusters will be re-detected by PRISM and thus provide an invaluable inter-calibration of X-ray and SZ effect cluster cosmology, provide determinations of cluster temperatures by combining the two detection techniques, and obtain independent

\footnotetext{
${ }^{5}$ http://www.ccatobservatory.org/index.cfm/page/index.htm.
} 
cluster distances for many thousands of clusters whose X-ray temperatures and shape parameters can be obtained from the X-ray survey. With $\sim 10^{6}$ clusters detected with PRISM, one can also further exploit the $e$ ROSITA survey data by stacking in a way similar to the analysis of the X-ray signals from the ROSAT All-Sky Survey for SDSS detected clusters [190].

Ambitious imaging surveys in the optical and near-IR will also produce large cluster surveys. These include the ground-based Dark Energy Survey (DES), ${ }^{6}$ now operating, and the future Large Synoptic Survey Telescope $(\operatorname{LSST})^{7}$ [130], a 8m-class telescope to be constructed in Chile and dedicated to a ten-year survey of the Southern Sky starting in 2020. The LSST final survey depth will reach $\sim 27$ magnitude in six bands (ugrizY), surpassing the DES depth of $\sim 24$ magnitude (in grizY bands). These broad-band imaging surveys will detect clusters out to redshifts of order unity through the characteristic colors of their member galaxies, which lie on the so-called "red sequence" in a color-magnitude diagram. The lensing measurements undertaken in these surveys will also produce mass calibrations for cluster scaling relations.

In addition, the European Space Agency's Euclid mission, scheduled for launch in 2020, will extend these cluster catalogs to higher redshifts thanks to its space-based IR observations $^{8}$ [119]. Euclid is estimated to detect between $(5-10) \times 10^{4}$ clusters out to redshifts well beyond unity. Moreover, Euclid's exquisite gravitational lensing survey will furnish a highquality mass calibration for the cluster scaling laws. Comparison of weak lensing and CMB lensing mass calibrations from PRISM will allow for the most robust possible calibration for these scaling relations that are crucial to cluster science. This comparison is possible in the overlap region at redshifts up to unity. Beyond $z \approx 1$ only CMB-based lensing by PRISM will be used to calibrate the scaling relations.

\subsection{Other sub-millimeter and far-infrared initiatives}

PRISM will map the full-sky, large-scale continuum emission at higher sensitivities than ground based single-dish telescopes operating in the same frequency range. Ground-based instruments can observe smaller regions with higher angular resolution than PRISM and are hence complementary. Existing (APEX, ASTE, IRAM 30m, LMT) and future (CCAT) ground-based single-dish sub-millimeter observatories are of interest for ground-based followup, although they are not as sensitive above $300 \mathrm{GHz}$ as PRISM (mainly because of the limitations of observing through the atmosphere).

CCAT will initially have two imaging instruments, LWCam and SWCam. At low frequencies, LWCam will be able to detect sources below the PRISM confusion limit relatively quickly. However variations in atmospheric transmissivity and thermal radiation from the atmosphere will make it difficult for $C C A T$ to map large scale structures. At high frequencies, $S W C a m$ will have difficulty mapping large areas to the confusion limit of PRISM. Based on the specifications from Stacey et al. (2013), CCAT can map an area of 1 square degree at 857 $\mathrm{GHz}$ to a sensitivity of $6 \mathrm{mJy}$ (the PRISM confusion limit) within 1 hour. To map the entire southern sky to this same depth however requires $\sim 900$ days $(24 \mathrm{~h})$ with optimal observing conditions. Such large scale observations will not be feasible with CCAT. Only a scanning space mission with a cold telescope such as PRISM can produce high sensitivity maps over a large area of sky in these frequency bands.

\footnotetext{
${ }^{6}$ https://www.darkenergysurvey.org/index.shtml.

${ }^{7}$ http://www.lsst.org/lsst/.

${ }^{8}$ http://www.euclid-ec.org.
} 
Interferometers ( $A L M A, C A R M A, P d B$ Interferometer, $S M A)$ are ill-suited to observing large fields and insensitive to large-scale structure, but can be used to produce superior maps of selected sky regions in combination with PRISM data. ALMA, operating in the range 30-1000 GHz, will complement PRISM with follow-up of sources and clusters to map their structure in total intensity, polarization and to observe spectral lines at high angular and spectral resolution.

Few previous infrared telescopes have performed all-sky surveys in the bands covered by PRISM. Akari was the last telescope to perform such observations, but the data is at much lower sensitivity and resolution and is not yet publicly available. Several other prior telescopes (Spitzer, Herschel) as well as the airborne observatory SOFIA have observed or will observe in the $600-4000 \mathrm{GHz}$ range, but only over very limited areas of the sky. Furthermore, except for a few deep fields, they observe objects already identified in other bands. PRISM will be able to perform observations with sensitivities comparable to Herschel or better, but covering the entire sky in many frequency bands.

\subsection{Other dark energy probes}

The question of Dark Energy will be investigated in depth before PRISM. Several dark energy experiments as well as a space mission are planned for the near future, among which DESI, LSST and Euclid stand out as among the most competitive. In spite of this vigorous observational program, PRISM is expected to still shed additional light on this most puzzling piece of our current cosmological model.

Building upon the expertise acquired with SDSS-III/BOSS, DESI is a ground-based dark-energy experiment that will study baryon acoustic oscillations and the growth of structure with a wide area (14000 square degrees) spectroscopic survey of approximately 20 million galaxies and 600 thousand quasars. The redshift of the targets (luminous red galaxies to $z=1$, emission line galaxies with $0.7<z<1.7$, and quasars with $1<z<5$ ) will be measured with the $4 \mathrm{~m}$ Mayall telescope at Kitt Peak (Arizona). DESI is expected to last from 2018 to 2024 and aims to measure the cosmic distance scale to $1 \%$ in 20 redshift bins from 0 to 3.5. The galaxy part of the survey will be limited only by cosmic variance.

The Large Synoptic Survey Telescope (LSST) is a ground-based photometric survey that will obtain multiple images of the sky with an $8 \mathrm{~m}$ telescope in Cerro Pachon, Northern Chile. During the anticipated 10 years of operation (approximately 2020-2030), LSST will uniformly observe a 18000 square degree region about 1000 times (summed over the 6 optical bands). The time information will be used to detect and measure light curves of over $10^{5}$ SNIa, while the deep co-added images will provide photometric redshifts of several billion galaxies. These data will be used together to provide a combined constrain on dark energy and the growth of structure from weak lensing, baryon acoustic oscillations and SNIa.

Unlike the previous two projects, Euclid is a space-based survey, resulting from the merging of a baryon acoustic oscillation redshift project (SPACE) and a weak lensing photometric project (DUNE) both proposed to ESA. The resulting survey will use these two techniques to constrain the dark universe using the measured redshifts of about 50 million galaxies over 15000 square degrees and the photometric information of about 20 billion sources. The launch date of the Euclid satellite is currently expected to be around 2020 .

A comparison of these projects was recently compiled by Font-Ribera et al. [75], who compared the expected constraints on the isotropic measurement of the cosmic distance scale from the spectroscopic DESI and Euclid BAO projects in addition to constraints from other earlier future or ongoing surveys. Font-Ribera et al. provide the following Dark Energy Task 
Force figures-of-merit $(\mathrm{FoM})$ for these projects (with the normalization FoM $=\left(\sigma_{w} \sigma_{w_{a}}\right)^{-1}$ ) including priors from Planck in each case: $\mathrm{FoM}=2$ for Planck alone, FoM from 23 to 104 for each of the individual dark energy probes of DESI, reaching 318 for an appropriate combination of DESI probes, FoM=27 for Euclid BAO, and FoM=134 for LSST or Euclid weak lensing. Combining the two Euclid primary probes yields a FoM of 430 [119].

It is difficult to foresee the state of Dark Energy research when PRISM will be launched. However in terms of the usual FoM, the constraints from the galaxy clusters observed by PRISM alone will outperform all the above observations (see figure 4), and also probe more complex models such as quintessence (section 3). Additional constraints will be obtained from PRISM primary CMB including lensing (figure 14). Moreover PRISM will significantly improve constraints on other cosmological parameters (section 6), which can be used to better exploit galaxy lensing and $\mathrm{BAO}$ oscillation data.

\subsection{SPICA}

The proposed Japanese space mission SPICA, originally scheduled for launch in the first half of the 2020s, addresses a few science objectives closely related to a part of the PRISM science program. In particular, the SAFARI instrument will carry out imaging and spectroscopic observations of the sky in the 34-200 micron range (instead of 50-10000 microns for PRISM) at the focus of a $\sim 3 \mathrm{~m}$ telescope cooled to about $5-6 \mathrm{~K}$. The two mission concepts, however, differ substantially.

The biggest difference is the fact that while SPICA will perform targeted observations towards a number of selected regions, the scientific program of PRISM requires observing the full sky in intensity and polarization. The broad-band sensitivity that can be achieved with a telescope cooled down to below $10 \mathrm{~K}$ is sufficient to guarantee that the PRISM survey will be confusion limited in its full frequency range. Therefore, even if SAFARI can in principle achieve better imaging sensitivity by spending more time integrating on a small patch of sky (figure 24), its actual point source detection performance, limited by confusion, will be comparable to what will be achieved by PRISM over the full sky. The maps from PRISM will thus extend to the entire sky the high quality and confusion limited observations obtained with the imaging mode of SAFARI on SPICA.

PRISM, however, cannot match SPICA's spectroscopic capabilities for detecting atomic and molecular lines in distant galaxies. The spectral resolution of the PRISM narrow-band imager is of order $R \approx 40$, about 50 times worse than the SAFARI spectroscopic mode. Hence, both missions will generate complementary observations of the distant universe, PRISM being clearly superior for detecting a large number of objects, SPICA being superior to observe selected objects in detail.

In our own galaxy, PRISM will map the main atomic and molecular lines to get a complete picture of the ISM on large scales, while SPICA will observe targeted regions in detail with similar angular resolution, but much higher spectral resolution. A key feature of PRISM, matched by no other existing or planned instrument including SPICA, and of much relevance to galactic astrophysics, is the capability of PRISM to observe polarized emission at sub-millimeter wavelengths. Polarization data is essential to untangle the role of the magnetic field for star formation in our Galaxy.

\subsection{Square Kilometer Array (SKA)}

The Square Kilometer Array (SKA) will span radio frequencies from $0.07 \mathrm{GHz}$ to $10-20$ $\mathrm{GHz}$, close to the PRISM frequency range, but with no overlap. It will observe more than 


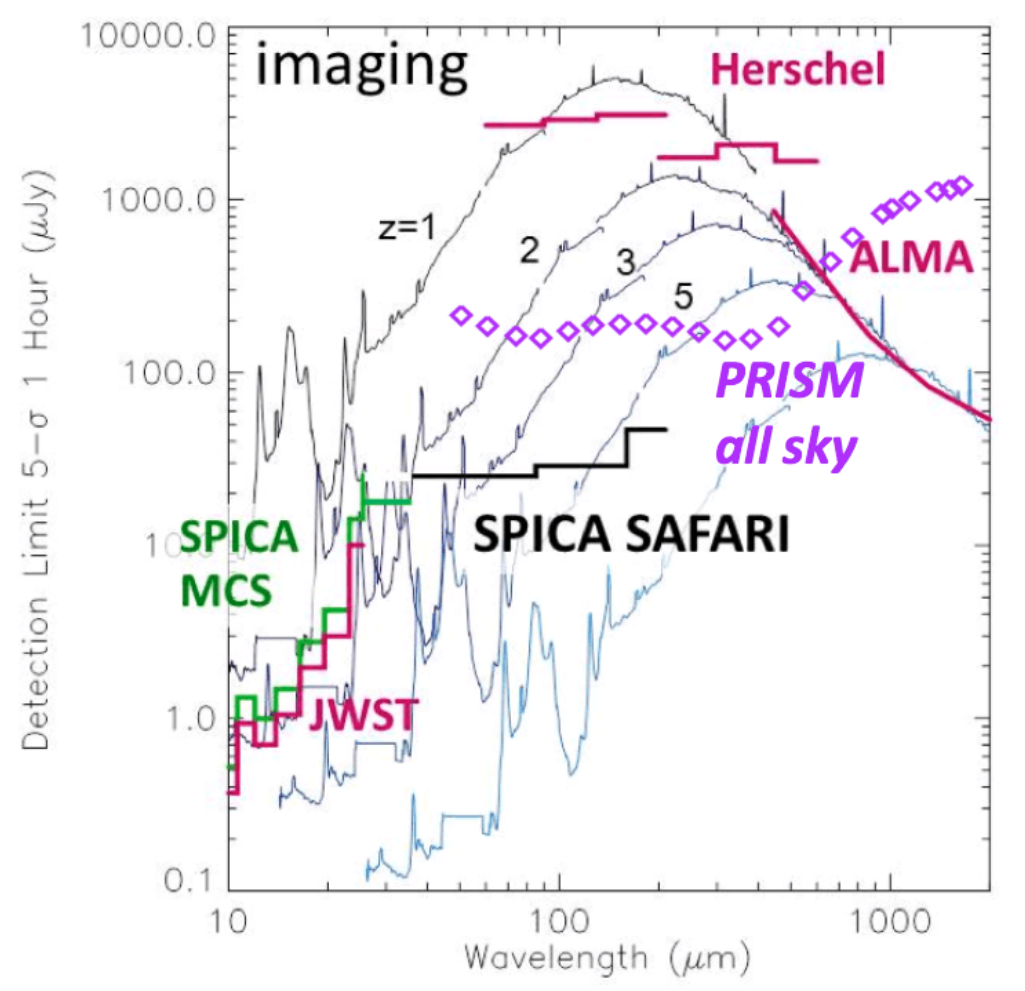

Figure 24. The full-sky point source sensitivity of the PRISM polarimetric imager compared to other existing or planned experiments. Each diamond corresponds to the sky-average point source sensitivity (excluding confusion) in a single frequency channel of PRISM. Note that PRISM is in fact confusion limited over the full frequency range, and thus the actual detection limit for both PRISM and SPICA will be similar, slightly higher than the PRISM limit (figure adapted from [140]). For illustrative purposes the SED of the starburst galaxy M82 as redshifted to the values indicated is shown in the background.

$10^{9}\left(f_{\text {sky }} / 0.5\right)$ HI galaxies over the redshift range $0<z<1.5$ and provide maps of the reionization epoch above $z \sim 6$, completing the PRISM view of structures in our Hubble volume. The capabilities of SKA will also enhance the range of investigations possible with the PRISM data set.

Definition of the high frequency reach of SKA Phase 1 is in progress, with a baseline design compatible with observations up to $14.5 \mathrm{GHz}$ at the sub-system level, and dish specifications for good performance up to $20 \mathrm{GHz}$. Capabilities for observing at the highest frequencies will be implemented around 2025 with the SKA Phase 2, and be fully operational at the foreseen PRISM launch date in 2028 or 2034.

Plans include operations over several decades, which will make follow-up observations of sources identified by PRISM possible, as well as detailed exploration of very faint emission on patches of the sky. One of the most relevant perspectives offered by the surface brightness sensitivity achievable with SKA's compact core array is the detailed mapping out to the virial radius of the majority of the $\sim 10^{6}$ clusters detected by PRISM. Such follow-up observations of the good statistical sample provided by PRISM down to low masses and up to high redshifts will enable the study of magnetic fields in clusters through Faraday rotation measurement of background sources and the study of cluster radio halos, as well as determinations of the dark matter distribution through lensing of background radio sources. 
These capabilities complement and enhance PRISM's, whose higher frequencies covering the zero-crossing of the SZ effect make PRISM more efficient at finding clusters, and allow measurement of the kSZ and relativistic SZ effects as well as exploiting CMB lensing for cluster mass determinations.

SKA will also complement PRISM by assessing the SZ effects expected from protogalactic gas heated at the virial temperature and from quasar driven blast-waves, making possible accurate prediction of the corresponding background of fluctuations on very small scales (at multipoles of several thousands).

The extreme SKA sensitivity will probe radio source counts down to hundreds or even tens of nJy, thus providing additional information about their contribution to the total radio background. This is relevant for the scientific exploitation of PRISM data to measure the absolute CMB and Galactic emission at the lowest PRISM frequencies. Furthermore, SKA will probe the free-free emission associated with cosmological reionization by looking at single sources and in a statistical sense, including the global integrated signal, thereby complementing PRISM spectrum measurements below $30 \mathrm{GHz}$.

SKA will contribute substantially to understanding galaxy formation and its evolution, AGN feedback, and star formation history. These observations complement the PRISM studies of extragalactic sources and the CIB with information over the radio spectrum. Similarly, the exquisite mapping of the Galactic radio foreground with SKA in both temperature and polarization will complement PRISM observations of the low-frequency galactic emission, with the characterization of the small scales not observed with the space-borne observatory.

\section{References}

[1] T. Essinger-Hileman et al., The Atacama B-Mode Search: CMB Polarimetry with Transition-Edge-Sensor Bolometers, arXiv:1008.3915 [INSPIRE].

[2] A. Avgoustidis et al., Constraints on the fundamental string coupling from B-mode experiments, Phys. Rev. Lett. 107 (2011) 121301 [arXiv:1105.6198] [INSPIRE].

[3] D. Barbosa, J.G. Bartlett, A. Blanchard and J. Oukbir, The Sunyaev-Zel'dovich effect and the value of $\Omega_{o}$, Astron. Astrophys. 314 (1996) 13 [astro-ph/9511084] [INSPIRE].

[4] R. Barkana and A. Loeb, In the beginning: The First sources of light and the reionization of the Universe, Phys. Rept. 349 (2001) 125 [astro-ph/0010468] [INSPIRE].

[5] J.D. Barrow and P. Coles, Primordial density fluctuations and the microwave background spectrum, Mon. Not. R. Astron. Soc. 248 (1991) 52.

[6] J.G. Bartlett, The Sunyaev-Zel'dovich Effect and its Uses, Asptrophys. Space Sci. 290 (2004) 105.

[7] J.G. Bartlett and J. Silk, A Comptonization model for the submillimeter background, Astrophys. J. 353 (1990) 399.

[8] S. Basak and J. Delabrouille, A needlet internal linear combination analysis of WMAP 7-year data: estimation of CMB temperature map and power spectrum, Mon. Not. R. Astron. Soc. 419 (2012) 1163.

[9] S. Basak and J. Delabrouille, A needlet ILC analysis of WMAP 9-year polarization data: CMB polarization power spectra, Mon. Not. R. Astron. Soc. 435 (2013) 18.

[10] K. Basu, C. Hernandez-Monteagudo and R. Sunyaev, CMB observations and the production of chemical elements at the end of the dark ages, Astron. Astrophys. 416 (2004) 447 [astro-ph/0311620] [INSPIRE]. 
[11] D Baumann et al., CMBPol Mission Concept Study: Probing Inflation with CMB Polarization, AIP Conf.Proc. 1141 (2009) 10.

[12] V.N. Bennert, M.W. Auger, T. Treu, J.-H. Woo and M.A. Malkan, The Relation between Black Hole Mass and Host Spheroid Stellar Mass out to z 2, Astrophys. J. 742 (2011) 107 [arXiv: 1102.1975] [INSPIRE].

[13] C.L. Bennett et al., First-Year Wilkinson Microwave Anisotropy Probe (WMAP) Observations: Preliminary Maps and Basic Results, Astrophys. J. Suppl. S. 148 (2003) 1.

[14] B. Benson et al., Cosmological Constraints from Sunyaev-Zel'dovich-Selected Clusters with X-ray Observations in the First 178 Square Degrees of the South Pole Telescope Survey, Astrophys. J. 763 (2013) 147 [arXiv:1112.5435] [INSPIRE].

[15] M. Béthermin, The redshift evolution of the distribution of star formation among dark matter halos as seen in the infrared, Astron. Astrophys. 557 (2013) A66.

[16] M. Biagetti, H. Perrier, A. Riotto and V. Desjacques, Testing the running of non-Gaussianity through the CMB $\mu$-distortion and the halo bias, Phys. Rev. D 87 (2013) 063521.

[17] H. Chiang et al., Measurement of CMB Polarization Power Spectra from Two Years of BICEP Data, Astrophys. J. 711 (2010) 1123 [arXiv:0906.1181] [INSPIRE].

[18] R.W. Ogburn et al., BICEP2 and Keck Array operational overview and status of observations, in Society of Photo-Optical Instrumentation Engineers (SPIE) Conference Series 8452 (2012).

[19] M. Birkinshaw, The Sunyaev-Zel'dovich effect, Phys. Rept. 310 (1999) 97 [astro-ph/9808050] [INSPIRE].

[20] L.E. Bleem et al., A Measurement of the Correlation of Galaxy Surveys with CMB Lensing Convergence Maps from the South Pole Telescope, Astrophys. J. Lett. 753 (2012) L9.

[21] COrE collaboration, F. Bouchet et al., COrE (Cosmic Origins Explorer) A White Paper, arXiv:1102.2181 [INSPIRE].

[22] P. Brax, C. Burrage, A.-C. Davis and G. Gubitosi, Cosmological Tests of the Disformal Coupling to Radiation, JCAP 11 (2013) 001 [arXiv:1306.4168] [INSPIRE].

[23] T. Bringmann, P. Scott and Y. Akrami, Improved constraints on the primordial power spectrum at small scales from ultracompact minihalos, Phys. Rev. D 85 (2012) 125027 [arXiv: 1110.2484] [INSPIRE].

[24] P. Bull and M. Kamionkowski, What if Planck's Universe isn't flat?, Phys. Rev. D 87 (2013) 081301 [arXiv: 1302.1617] [INSPIRE].

[25] C. Burigana, Distortions of the CMB Spectrum by Continuous Heating, in G.L. Chincarini, A. Iovino, T. Maccacaro and D. Maccagni eds., Observational Cosmology, Astronomical Society of the Pacific Conference Series 51 (1993) 554.

[26] C. Burigana, L. Danese and G. de Zotti, Formation and evolution of early distortions of the microwave background spectrum - A numerical study, Astron. Astrophys. 246 (1991) 49.

[27] Z.-Y. Cai et al., A hybrid model for the evolution of galaxies and Active Galactic Nuclei in the infrared, Astrophys. J. 768 (2013) 21 [arXiv:1303.2335] [INSPIRE].

[28] R. Caldwell and N. Maksimova, Spectral Distortion in a Radially Inhomogeneous Cosmology, arXiv:1309.4454 [INSPIRE].

[29] B. Carr, K. Kohri, Y. Sendouda and J. Yokoyama, New cosmological constraints on primordial black holes, Phys. Rev. D 81 (2010) 104019 [arXiv:0912.5297] [INSPIRE].

[30] R. Cen and J.P. Ostriker, Where are the baryons?, Astrophys. J. 514 (1999) 1 [astro-ph/9806281] [INSPIRE]. 
[31] R. Cen and J.P. Ostriker, Where are the baryons? 2. feedback effects, Astrophys. J. 650 (2006) 560 [astro-ph/0601008] [INSPIRE].

[32] A. Challinor and A. Lasenby, Relativistic Corrections to the Sunyaev-Zeldovich Effect, Astrophys. J. 499 (1998) 1.

[33] J. Chluba, Spectral Distortions of the Cosmic Microwave Background, Ph.D. thesis, LMU München, Germany, March 2005.

[34] J. Chluba, Could the cosmological recombination spectrum help us understand annihilating dark matter?, Mon. Not. R. Astron. Soc. 402 (2010) 1195.

[35] J. Chluba, Distinguishing different scenarios of early energy release with spectral distortions of the cosmic microwave background, arXiv:1304.6121 [INSPIRE].

[36] J. Chluba, Green's function of the cosmological thermalization problem, Mon. Not. R. Astron. Soc. 434 (2013) 352.

[37] J. Chluba, A.L. Erickcek and I. Ben-Dayan, Probing the inflaton: Small-scale power spectrum constraints from measurements of the CMB energy spectrum, Astrophys. J. 758 (2012) 76 [arXiv: 1203.2681] [INSPIRE].

[38] J. Chluba and D. Grin, CMB spectral distortions from small-scale isocurvature fluctuations, Mon. Not. R. Astron. Soc. 434 (2013) 1619.

[39] J. Chluba and D. Jeong, Teasing bits of information out of the CMB energy spectrum, arXiv: 1306.5751 [INSPIRE].

[40] J. Chluba, R. Khatri and R.A. Sunyaev, $C M B$ at $2 \times 2$ order: the dissipation of primordial acoustic waves and the observable part of the associated energy release, Mon. Not. R. Astron. Soc. 425 (2012) 1129.

[41] J. Chluba and R.A. Sunyaev, Free-bound emission from cosmological hydrogen recombination, Astron. Astrophys. 458 (2006) L29.

[42] J. Chluba and R.A. Sunyaev, Pre-recombinational energy release and narrow features in the CMB spectrum, Astron. Astrophys. 501 (2009) 29.

[43] J. Chluba and R.A. Sunyaev, The evolution of CMB spectral distortions in the early Universe, Mon. Not. R. Astron. Soc. 419 (2012) 1294.

[44] S. Chongchitnan and G. Efstathiou, Dynamics of the inflationary flow equations, Phys. Rev. D 72 (2005) 083520 [astro-ph/0508355] [INSPIRE].

[45] S. Colafrancesco, SZ effect from dark matter annihilation, Astron. Astrophys. 422 (2004) L23 [astro-ph/0405456] [INSPIRE].

[46] S. Colafrancesco, The SZ effect from cluster cavities, Astron. Astrophys. 435 (2005) L9 [astro-ph/0503646] [INSPIRE].

[47] S. Colafrancesco, $S Z$ effect from radio-galaxy lobes: astrophysical and cosmological relevance, Mon. Not. R. Astron. Soc. 385 (2008) 2041.

[48] S. Colafrancesco and P. Marchegiani, On the ability of the spectroscopic Sunyaev-Zeldovich effect measurements to determine the temperature structure of galaxy clusters, Astron. Astrophys. 520 (2010) A31.

[49] S. Colafrancesco, P. Marchegiani and R. Buonanno, Untangling the atmosphere of the Bullet cluster with Sunyaev-Zel'dovich effect observations, Astron. Astrophys. 527 (2011) L1.

[50] S. Colafrancesco, P. Marchegiani and E. Palladino, The Non-thermal Sunyaev - Zel'dovich effect in clusters of galaxies, Astron. Astrophys. 397 (2003) 27 [astro-ph/0211649] [INSPIRE]. 
[51] S. Colafrancesco, D. Prokhorov and V. Dogiel, Studying the leptonic structure of galaxy cluster atmospheres from the spectral properties of the SZ effect, Astron. Astrophys. 494 (2009) 1 [arXiv:0905.2752] [INSPIRE].

[52] R.M. Crutcher, Magnetic Fields in Molecular Clouds, Annu. Rev. Astron. Astr. 50 (2012) 29.

[53] A.C.d. Silva, D. Barbosa, A.R. Liddle and P.A. Thomas, Hydrodynamical simulations of the sunyaev-zel'dovich effect: the kinetic effect, Mon. Not. Roy. Astron. Soc. 326 (2001) 155 [astro-ph/0011187] [INSPIRE].

[54] R.A. Daly, Spectral distortions of the microwave background radiation resulting from the damping of pressure waves, Astrophys. J. 371 (1991) 14.

[55] L. Danese and C. Burigana, Theoretical Aspects of the CMB Spectrum, in J.L. Sanz, E. Martinez-Gonzalez and L. Cayon eds., Present and Future of the Cosmic Microwave Background, Lect. Notes Phys. 429 (1994) 28.

[56] L. Danese and G. de Zotti, The relic radiation spectrum and the thermal history of the Universe, Nuovo Cimento 7 (1977) 277.

[57] S. Das et al., The Atacama Cosmology Telescope: Temperature and Gravitational Lensing Power Spectrum Measurements from Three Seasons of Data (2013), arXiv:1301.1037 [INSPIRE].

[58] S. Das et al., Detection of the Power Spectrum of Cosmic Microwave Background Lensing by the Atacama Cosmology Telescope, Phys. Rev. Lett. 107 (2011) 021301 [arXiv:1103.2124] [INSPIRE].

[59] P. de Bernardis et al., Low-resolution spectroscopy of the Sunyaev-Zel'dovich effect and estimates of cluster parameters, Astron. Astrophys. 538 (2012) A86 [arXiv:1111.4588] [INSPIRE].

[60] J. Delabrouille et al., The pre-launch Planck Sky Model: a model of sky emission at submillimetre to centimetre wavelengths, Astron. Astrophys. 553 (2013) A96.

[61] J. Delabrouille, J.-F. Cardoso, M. Le Jeune, M. Betoule, G. Fay and F. Guilloux, A full sky, low foreground, high resolution CMB map from WMAP, Astron. Astrophys. 493 (2009) 835.

[62] J. Delabrouille, J.-B. Melin and J.G. Bartlett, Simulations of Sunyaev-Zel'dovich Maps and Their Applications, in L.-W. Chen, C.-P. Ma, K.-W. Ng and U.-L. Pen eds., AMiBA 2001: High-Z Clusters, Missing Baryons, and CMB Polarization, Astronomical Society of the Pacific Conference Series 257 (2002) 81.

[63] J.B. Dent, D.A. Easson and H. Tashiro, Cosmological constraints from CMB distortion, Phys. Rev. D 86 (2012) 023514 [arXiv: 1202.6066] [INSPIRE].

[64] K. Dolag, M. Meneghetti, L. Moscardini, E. Rasia and A. Bonaldi, Simulating the physical properties of dark matter and gas inside the cosmic web, Mon. Not. R. Astron. Soc. 370 (2006) 656.

[65] B.T. Draine, Interstellar Dust Models and Evolutionary Implications, in T. Henning, E. Grün and J. Steinacker eds., Cosmic dust: Near and far, Astronomical Society of the Pacific Conference Series 414 (2009) 453.

[66] B. Draine and B. Hensley, Magnetic Nanoparticles in the Interstella Medium: Emission Spectrum and Polarization, Astrophys. J. 765 (2013) 159 [arXiv:1205.7021] [INSPIRE].

[67] V.K. Dubrovich, Hydrogen recombination lines of cosmological origin, Sov. Ast. Lett. 1 (1975) 196.

[68] C. Dvorkin and W. Hu, Generalized Slow Roll for Large Power Spectrum Features, Phys. Rev. D 81 (2010) 023518 [arXiv:0910.2237] [INSPIRE]. 
[69] R. Easther and H. Peiris, Implications of a Running Spectral Index for Slow Roll Inflation, JCAP 09 (2006) 010 [astro-ph/0604214] [INSPIRE].

[70] B. Reichborn-Kjennerud et al., EBEX: a balloon-borne CMB polarization experiment, Society of Photo-Optical Instrumentation Engineers (SPIE) Conference Series 7741 (2010).

[71] J.L. Feng, Dark Matter Candidates from Particle Physics and Methods of Detection, Annu. Rev. Astron. Astr. 48 (2010) 495.

[72] J.L. Feng, A. Rajaraman and F. Takayama, SuperWIMP dark matter signals from the early universe, Phys. Rev. D 68 (2003) 063504 [hep-ph/0306024] [InSPIRE].

[73] D.P. Finkbeiner and N. Weiner, Exciting Dark Matter and the INTEGRAL/SPI $511 \mathrm{keV}$ signal, Phys. Rev. D 76 (2007) 083519 [astro-ph/0702587] [INSPIRE].

[74] D. Fixsen et al., The Cosmic Microwave Background spectrum from the full COBE FIRAS data set, Astrophys. J. 473 (1996) 576 [astro-ph/9605054] [INSPIRE].

[75] A. Font-Ribera, P. McDonald, N. Mostek, B.A. Reid, H.-J. Seo and A. Slosar, DESI and other dark energy experiments in the era of neutrino mass measurements, arXiv:1308.4164 [INSPIRE].

[76] A. Fraisse et al., SPIDER: Probing the Early Universe with a Suborbital Polarimeter, JCAP 04 (2013) 047 [arXiv:1106 . 3087] [INSPIRE].

[77] S. Galli et al., Constraining Fundamental Physics with Future CMB Experiments, Phys. Rev. D 82 (2010) 123504 [arXiv: 1005.3808] [INSPIRE].

[78] J. Ganc and E. Komatsu, Scale-dependent bias of galaxies and mu-type distortion of the cosmic microwave background spectrum from single-field inflation with a modified initial state, Phys. Rev. D 86 (2012) 023518 [arXiv:1204.4241] [INSPIRE].

[79] J. Geach et al., A Direct Measurement of the Linear Bias of Mid-infrared-selected Quasars at $z \approx 1$ Using Cosmic Microwave Background Lensing, Astrophys. J. 776 (2013) L41 [arXiv:1307.1706] [INSPIRE].

[80] P. Goldreich and N.D. Kylafis, On mapping the magnetic field direction in molecular clouds by polarization measurements, Astrophys. J. Lett. 243 (1981) L75.

[81] Y. Gong, A. Cooray, M. Silva, M.G. Santos, J. Bock, C.M. Bradford and M. Zemcov, Intensity Mapping of the [CII] Fine Structure Line during the Epoch of Reionization, Astrophys. J. $\mathbf{7 4 5}$ (2012) 49 [arXiv:1107.3553] [INSPIRE].

[82] M. Griffin et al., The Herschel-SPIRE instrument and its in-flight performance, Astron. Astrophys. 518 (2010) L3 [arXiv: 1005.5123] [INSPIRE].

[83] A.C. Hall and A. Challinor, Probing the neutrino mass hierarchy with cosmic microwave background weak lensing, Mon. Not. R. Astron. Soc. 425 (2012) 1170.

[84] S. Hannestad and T. Tram, Sommerfeld Enhancement of DM Annihilation: Resonance Structure, Freeze-Out and CMB Spectral Bound, JCAP 01 (2011) 016 [arXiv:1008.1511] [INSPIRE].

[85] SPTpol collaboration, D. Hanson et al., Detection of B-mode Polarization in the Cosmic Microwave Background with Data from the South Pole Telescope, Phys. Rev. Lett. 111 (2013) 141301 [arXiv: 1307.5830] [INSPIRE].

[86] M. Hilton et al., The Atacama Cosmology Telescope: the stellar content of galaxy clusters selected using the Sunyaev-Zel'dovich effect, arXiv:1301.0780 [INSPIRE].

[87] P. Hennebelle and E. Falgarone, Turbulent molecular clouds, Astron. Astrophys. Rev. 20 (2012) 55 [arXiv:1211.0637].

[88] C. Hernández-Monteagudo, Z. Haiman, R. Jimenez and L. Verde, Oxygen Pumping: Probing Intergalactic Metals at the Epoch of Reionization, Astrophys. J. Lett. 660 (2007) L85. 
[89] C. Hernández-Monteagudo, J.A. Rubiño-Martín and R.A. Sunyaev, On the influence of resonant scattering on cosmic microwave background polarization anisotropies, Mon. Not. R. Astron. Soc. 380 (2007) 1656.

[90] C. Hernandez-Monteagudo and R. Sunyaev, Cross terms and weak frequency dependent signals in the CMB sky, Mon. Not. Roy. Astron. Soc. 359 (2005) 597 [astro-ph/0405487] [INSPIRE].

[91] C. Hernandez-Monteagudo, L. Verde and R. Jimenez, Tomography of the Reionization Epoch with Multifrequency CMB Observations, Astrophys. J. 653 (2006) 1 [astro-ph/0604324] [INSPIRE].

[92] C. Hernandez-Monteagudo, L. Verde, R. Jimenez and D.N. Spergel, Correlation properties of the kinematic sunyaev-zel'dovich effect and implications for dark energy, Astrophys. J. 643 (2006) 598 [astro-ph/0511061] [INSPIRE].

[93] H. Hildebrandt et al., Inferring the mass of submillimetre galaxies by exploiting their gravitational magnification of background galaxies, Mon. Not. R. Astron. Soc. 429 (2013) 3230.

[94] C.M. Hirata, S. Ho, N. Padmanabhan, U. Seljak and N.A. Bahcall, Correlation of CMB with large-scale structure: II. Weak lensing, Phys. Rev. D 78 (2008) 043520 [arXiv:0801.0644] [INSPIRE].

[95] G. Holder et al., A Cosmic Microwave Background Lensing Mass Map and Its Correlation with the Cosmic Infrared Background, Astrophys. J. 771 (2013) L16 [arXiv:1303.5048] [INSPIRE].

[96] W. Hu, D. Scott and J. Silk, Power spectrum constraints from spectral distortions in the cosmic microwave background, Astrophys. J. 430 (1994) L5 [astro-ph/9402045] [INSPIRE].

[97] W. Hu, D. Scott and J. Silk, Reionization and cosmic microwave background distortions: A Complete treatment of second order Compton scattering, Phys. Rev. D 49 (1994) 648 [astro-ph/9305038] [INSPIRE].

[98] W. Hu and J. Silk, Thermalization and spectral distortions of the cosmic background radiation, Phys. Rev. D 48 (1993) 485 [inSPIRE].

[99] W. Hu and J. Silk, Thermalization constraints and spectral distortions for massive unstable relic particles, Phys. Rev. Lett. 70 (1993) 2661 [InSPIRE].

[100] W. Hu and N. Sugiyama, Thermal history constraints on the isocurvature baryon model, Astrophys. J. 436 (1994) 456 [astro-ph/9403031] [INSPIRE].

[101] W. Hu, Mapping the dark matter through the CMB damping tail, Astrophys. J. 557 (2001) L79 [astro-ph/0105424] [INSPIRE].

[102] W. Hu, Dark synergy: Gravitational lensing and the CMB, Phys. Rev. D 65 (2002) 023003 [astro-ph/0108090] [INSPIRE].

[103] W. Hu and T. Okamoto, Mass reconstruction with CMB polarization, Astrophys. J. 574 (2002) 566 [astro-ph/0111606] [INSPIRE].

[104] A.F. Illarionov and R.A. Sunyaev, Comptonization, the spectrum of RELICT radiation, and the thermal history of the universe, Astronomicheskii Zhurnal 51 (1974) 1162.

[105] N. Itoh, Y. Kohyama and S. Nozawa, Relativistic Corrections to the Sunyaev-Zel'dovich Effect for Clusters of Galaxies, Astrophys. J. 502 (1998) 7 [astro-ph/9712289] [INSPIRE].

[106] R. Ivison et al., Herschel-ATLAS: A Binary HyLIRG Pinpointing a Cluster of Starbursting Protoellipticals, Astrophys. J. 772 (2013) 137 [arXiv:1302.4436] [INSPIRE].

[107] K. Jedamzik, V. Katalinic and A.V. Olinto, A Limit on primordial small scale magnetic fields from CMB distortions, Phys. Rev. Lett. 85 (2000) 700 [astro-ph/9911100] [InSPIRE].

[108] M. Kawasaki, K. Kohri and T. Moroi, Big-Bang nucleosynthesis and hadronic decay of long-lived massive particles, Phys. Rev. D 71 (2005) 083502 [astro-ph/0408426] [INSPIRE]. 
[109] R. Khatri and R.A. Sunyaev, Beyond $y$ and $\mu$ : the shape of the CMB spectral distortions in the intermediate epoch, JCAP 09 (2012) 016 [arXiv: 1207.6654] [INSPIRE].

[110] R. Khatri and R.A. Sunyaev, Creation of the CMB spectrum: precise analytic solutions for the blackbody photosphere, JCAP 06 (2012) 038 [arXiv:1203.2601] [INSPIRE].

[111] R. Khatri and R.A. Sunyaev, Forecasts for $C M B \mu$ and $i$-type spectral distortion constraints on the primordial power spectrum on scales $8 \lesssim k \lesssim 10^{4} \mathrm{Mpc}^{-1}$ with the future Pixie-like experiments, JCAP 06 (2013) 026 [arXiv:1303.7212] [INSPIRE].

[112] R. Khatri, R.A. Sunyaev and J. Chluba, Does Bose-Einstein condensation of CMB photons cancel $\mu$ distortions created by dissipation of sound waves in the early Universe?, Astron. Astrophys. 540 (2012) A124 [arXiv:1110.0475] [INSPIRE].

[113] R. Khatri, R.A. Sunyaev and J. Chluba, Mixing of blackbodies: entropy production and dissipation of sound waves in the early Universe, Astron. Astrophys. 543 (2012) A136 [arXiv: 1205.2871] [INSPIRE].

[114] R. Khatri and B.D. Wandelt, Crinkles in the last scattering surface: Non-Gaussianity from inhomogeneous recombination, Phys. Rev. D 79 (2009) 023501 [arXiv:0810.4370] [INSPIRE].

[115] W.H. Kinney, Inflation: Flow, fixed points and observables to arbitrary order in slow roll, Phys. Rev. D 66 (2002) 083508 [astro-ph/0206032] [INSPIRE].

[116] L. Kuzmin, G. Yassin, S. Withington and P. Grimes, An Antenna Coupled Cold-Electron Bolometer for High Performance Cosmology Instruments, in A. Karpov ed., Eighteenth International Symposium on Space Terahertz Technology (2007) 93.

[117] G. Lagache, J.-L. Puget and H. Dole, Dusty Infrared Galaxies: Sources of the Cosmic Infrared Background, Annu. Rev. Astron. Astr. 43 (2005) 727.

[118] A. Lapi et al., Herschel-ATLAS Galaxy Counts and High Redshift Luminosity Functions: The Formation of Massive Early Type Galaxies, Astrophys. J. 742 (2011) 24 [arXiv:1108.3911] [INSPIRE].

[119] EUCLID collaboration, R. Laureijs et al., Euclid Definition Study Report, arXiv:1110.3193 [INSPIRE].

[120] C.A. Leal-Sevillano, G. Pisano, J.R. Montejo-Garai, B. Maffei, J.A. Ruiz-Cruz, M.W. Ng and J.M. Rebollar, Development of Low Loss Waveguide Filters for Radio-Astronomy Applications, Infrared Phys. Techn. 61 (2013) 224.

[121] J.-L. Lehners, Ekpyrotic Non-Gaussianity: A Review, Adv. Astron. 2010 (2010) 903907 [arXiv: 1001.3125] [INSPIRE].

[122] C. Leinert et al., The 1997 reference of diffuse night sky brightness, Astron. Astrophys. Sup 127 (1998) 1.

[123] A. Lewis, Rayleigh scattering: blue sky thinking for future CMB observations, JCAP 08 (2013) 053 [arXiv: 1307.8148] [INSPIRE].

[124] A. Lewis, J. Weller and R. Battye, The cosmic microwave background and the ionization history of the Universe, Mon. Not. R. Astron. Soc. 373 (2006) 561.

[125] A. Lewis and A. Challinor, Weak gravitational lensing of the CMB, Phys. Rept. 429 (2006) 1 [astro-ph/0601594] [INSPIRE].

[126] J.E. Lidsey, A.R. Liddle, E.W. Kolb, E.J. Copeland, T. Barreiro and M. Abney, Reconstructing the inflaton potential-an overview, Rev. Mod. Phys. 69 (19970 373.

[127] K. Lochan, S. Das and A. Bassi, Constraining CSL strength parameter $\lambda$ from standard cosmology and spectral distortions of CMBR, Phys. Rev. D 86 (2012) 065016 [arXiv: 1206.4425] [INSPIRE]. 
[128] A. Loeb, Probing the Universe after Cosmological Recombination through the Effect of Neutral Lithium on the Microwave Background Anisotropies, Astrophys. J. Lett. 555 (2001) L1

[129] LSPE collaboration, F. Piacentini et al., The LSPE expected performance, talk given at 47th ESLAB conference "Universe as seen by Planck", April 2013, http://www.rssd.esa.int/index.php?project=PLANCK\&page=47_ESLAB.

[130] LSST ScIEnCE collaboration, P.A. Abell et al., LSST Science Book, Version 2.0, arXiv:0912.0201.

[131] Y.E. Lyubarsky and R.A. Sunyaev, The spectral features in the microwave background spectrum due to energy release in the early universe, Astron. Astrophys. 123 (1983) 171.

[132] C.-P. Ma, R. Caldwell, P. Bode and L.-M. Wang, The mass power spectrum in quintessence cosmological models, Astrophys. J. 521 (1999) L1 [astro-ph/9906174] [INSPIRE].

[133] J.C. Mather et al., Measurement of the Cosmic Microwave Background spectrum by the COBE FIRAS instrument, Astrophys. J. 420 (1994) 439 [INSPIRE].

[134] P. McDonald, R.J. Scherrer and T.P. Walker, Cosmic microwave background constraint on residual annihilations of relic particles, Phys. Rev. D 63 (2001) 023001 [astro-ph/0008134] [INSPIRE].

[135] J.J. McMahon et al., SPTpol: an instrument for CMB polarization, American Institute of Physics Conference Series 1185 (2009) 511.

[136] M. McQuinn, S.R. Furlanetto, L. Hernquist, O. Zahn and M. Zaldarriaga, The Kinetic Sunyaev-Zel'dovich effect from reionization, Astrophys. J. 630 (2005) 643 [astro-ph/0504189] [INSPIRE].

[137] M. McQuinn, A. Lidz, O. Zahn, S. Dutta, L. Hernquist and M. Zaldarriaga, The morphology of HII regions during reionization, Mon. Not. R. Astron. Soc. 377 (2007) 1043.

[138] J.-B. Melin, J.G. Bartlett and J. Delabrouille, Catalog extraction in SZ cluster surveys: a matched filter approach, Astron. Astrophys. 459 (2006) 341.

[139] F. Miniati, D. Ryu, H. Kang, T.W. Jones, R. Cen and J.P. Ostriker, Properties of Cosmic Shock Waves in Large-Scale Structure Formation, Astrophys. J. 542 (2000) 608.

[140] T. Nakagawa, H. Matsuhara and Y. Kawakatsu, The next-generation infrared space telescope SPICA, Society of Photo-Optical Instrumentation Engineers (SPIE) Conference Series $\mathbf{8 4 4 2}$ (2012).

[141] A. Natarajan, N. Battaglia, H. Trac, U.-L. Pen and A. Loeb, Reionization on Large Scales II: Detecting Patchy Reionization through Cross Correlation of the Cosmic Microwave Background, Astrophys. J. 776 (2013) 82 [arXiv:1211.2822] [InSPIRE].

[142] M. Negrello et al., The Detection of a Population of Submillimeter-Bright, Strongly Lensed Galaxies, Science 330 (2010) 800.

[143] M.D. Niemack et al., ACTPol: a polarization-sensitive receiver for the Atacama Cosmology Telescope, Society of Photo-Optical Instrumentation Engineers (SPIE) Conference Series 7741 (2010).

[144] S. Nozawa, N. Itoh and Y. Kohyama, Relativistic Thermal Bremsstrahlung Gaunt Factor for the Intracluster Plasma, Astrophys. J. 507 (1998) 530 [astro-ph/9802096] [INSPIRE].

[145] S. Nozawa, N. Itoh, Y. Suda and Y. Ohhata, An improved formula for the relativistic corrections to the kinematical Sunyaev-Zeldovich effect for clusters of galaxies, Nuovo Cimento B 121 (2006) 487.

[146] S.P. Oh, A. Cooray and M. Kamionkowski, Sunyaev-Zeldovich fluctuations from the first stars?, Mon. Not. R. Astron. Soc. 342 (2003) L20. 
[147] J.P. Ostriker and C. Thompson, Distortion of the cosmic background radiation by superconducting strings, Astrophys. J. Lett. 323 (1987) L97.

[148] E. Pajer and M. Zaldarriaga, New Window on Primordial Non-Gaussianity, http://dx.doi.org/10.1103/PhysRevLett.109.021302 Phys. Revs. Lett. 109 (2012) 021302.

[149] P. Pani and A. Loeb, Constraining Primordial Black-Hole Bombs through Spectral Distortions of the Cosmic Microwave Background, Phys. Rev. D 88 (2013) 041301 [arXiv:1307.5176] [INSPIRE].

[150] P. Peebles, Recombination of the Primeval Plasma, Astrophys. J. 153 (1968) 1 [INSPIRE].

[151] M. . Peel, C. Dickinson, R.D. Davies, D.L. Clements and R.J. Beswick, Radio to infrared spectra of late-type galaxies with Planck and Wilkinson Microwave Anisotropy Probe data, Mon. Not. R. Astron. Soc. 416 (2011) L99.

[152] WMAP collaboration, H. Peiris et al., First year Wilkinson Microwave Anisotropy Probe (WMAP) observations: Implications for inflation, Astrophys. J. Suppl. 148 (2003) 213 [astro-ph/0302225] [INSPIRE].

[153] A. Penin et al., An accurate measurement of the anisotropies and mean level of the Cosmic Infrared Background at 100 and 160 um, Astron. Astrophys. 543 (2012) A123 [arXiv: 1105.1463] [INSPIRE].

[154] J.L. Pineda, W.D. Langer, T. Velusamy and P.F. Goldsmith, A Herschel [CII] Galactic plane survey I: the global distribution of ISM gas components, arXiv:1304.7770 [INSPIRE].

[155] PIPER collaboration, D.T. Chuss et al., The Primordial Inflation Polarization Explorer (PIPER), Society of Photo-Optical Instrumentation Engineers (SPIE) Conference Series 7741 (2010).

[156] Planck collaboration, J. Aatrokoski et al., Planck early results. XV. Spectral energy distributions and radio continuum spectra of northern extragalactic radio sources, Astron. Astrophys. 536 (2011) A15.

[157] PLAnCK collaboration, P.A.R. Ade et al., Planck early results. XIV. ERCSC validation and extreme radio sources, Astron. Astrophys. 536 (2011) A14.

[158] Planck collaboration, P. Ade et al., Planck 2013 results. XIV. Zodiacal emission, arXiv:1303.5074 [INSPIRE].

[159] Planck collaboration, P. Ade et al., Planck 2013 results. XVI. Cosmological parameters, arXiv:1303.5076 [INSPIRE].

[160] Planck collaboration, P. Ade et al., Planck 2013 results. XVII. Gravitational lensing by large-scale structure, arXiv:1303.5077 [INSPIRE].

[161] Planck collaboration, P. Ade et al., Planck 2013 results. XVIII. Gravitational lensing-infrared background correlation, arXiv:1303.5078 [INSPIRE].

[162] Planck collaboration, P. Ade et al., Planck 2013 results. XX. Cosmology from Sunyaev-Zeldovich cluster counts, arXiv:1303.5080 [INSPIRE].

[163] Planck collaboration, P. Ade et al., Planck 2013 results. XXI. Cosmology with the all-sky Planck Compton parameter y-map, arXiv:1303.5081 [INSPIRE].

[164] Planck collaboration, P. Ade et al., Planck 2013 results. XXII. Constraints on inflation, arXiv:1303.5082 [INSPIRE].

[165] Planck collaboration, P. Ade et al., Planck 2013 Results. XXIV. Constraints on primordial non-Gaussianity, arXiv: 1303.5084 [INSPIRE].

[166] Planck collaboration, P. Ade et al., Planck 2013 results. XXV. Searches for cosmic strings and other topological defects, arXiv:1303.5085 [INSPIRE]. 
[167] Planck collaboration, P. Ade et al., Planck intermediate results. VIII. Filaments between interacting clusters, arXiv:1208.5911.

[168] Planck collaboration, P. Ade et al., Planck Intermediate Results. XI: The gas content of dark matter halos: the Sunyaev-Zeldovich-stellar mass relation for locally brightest galaxies, arXiv: 1212.4131 [INSPIRE].

[169] Planck collaboration, P. Ade et al., Planck Early Results XVIII: The power spectrum of cosmic infrared background anisotropies, Astron. Astrophys. 536 (2011) A18 [arXiv: 1101.2028] [INSPIRE].

[170] Planck collaboration, N. Aghanim et al., Planck early results. XII. Cluster Sunyaev-Zeldovich optical scaling relations, Astron. Astrophys. 536 (2011) A12

[171] POLARBEAR collaboration, A.Lee et al., The POLARBEAR CMB polarization experiment, talk given at 4 7th ESLAB conference "Universe as seen by Planck", April 2013, http://www.rssd.esa.int/index.php?project=PLANCK\&page=47_ESLAB.

[172] P. Ponente, J. Diego, R. Sheth, C. Burigana, S.R. Knollmann and Y. Ascasibar, The cosmological free-free signal from galaxy groups and clusters, Mon. Not. Roy. Astron. Soc. 410 (2011) 2353 [arXiv: 1006.2243] [INSPIRE].

[173] M. Pospelov and A. Ritz, The galactic 511 keV line from electroweak scale WIMPs, Phys. Lett. B 651 (2007) 208 [hep-ph/0703128] [INSPIRE].

[174] B.A. Powell, Scalar runnings and a test of slow roll from CMB distortions, arXiv:1209.2024 [INSPIRE].

[175] PRISM collaboration, P. Andre et al., PRISM (Polarized Radiation Imaging and Spectroscopy Mission): A White Paper on the Ultimate Polarimetric Spectro-Imaging of the Microwave and Far-Infrared Sky, arXiv:1306.2259 [INSPIRE].

[176] D.A. Prokhorov, S. Colafrancesco, T. Akahori, K. Yoshikawa, S. Nagataki and K.-I. Seon, Can electron distribution functions be derived through the Sunyaev-Zel'dovich effect?, Astron. Astrophys. 529 (2011) A39.

[177] QUBIC collaboration, J. Hamilton et al., QUBIC: The QU bolometric interferometer for cosmology, talk given at 4 7th ESLAB conference "Universe as seen by Planck", April 2013, http://www.rssd.esa.int/index.php?project=PLANCK\&page=47_ESLAB.

[178] QUIET collaboration, D. Araujo et al., Second Season QUIET Observations: Measurements of the CMB Polarization Power Spectrum at 95 GHz, Astrophys. J. 760 (2012) 145 [arXiv: 1207.5034] [INSPIRE].

[179] QUIJOTE collaboration, R. Rebolo et al., QUIJOTE: a CMB polarization experiment, talk given at 4 'th ESLAB conference "Universe as seen by Planck", April 2013, http://www.rssd.esa.int/index.php?project=PLANCK\&page=47_ESLAB.

[180] E.P.R.G. Ramos, A.J.C. da Silva and G.-C. Liu, Cosmic Microwave Background Induced Polarization from Single Scattering by Clusters of Galaxies and Filaments, Astrophys. J. $\mathbf{7 5 7}$ (2012) 44.

[181] A. Refregier, E. Komatsu, D.N. Spergel and U.-L. Pen, Power spectrum of the Sunyaev-Zel'dovich effect, Phys. Rev. D 61 (2000) 123001 [astro-ph/9912180] [InSPIRE].

[182] C. Reichardt et al., Galaxy clusters discovered via the Sunyaev-Zel'dovich effect in the first 720 square degrees of the South Pole Telescope survey, Astrophys. J. 763 (2013) 127 [arXiv:1203.5775] [INSPIRE].

[183] Y. Rephaeli, Cosmic microwave background comptonization by hot intracluster gas, Astrophys. J. 445 (1995) 33. 
[184] Y. Rephaeli and O. Lahav, Peculiar cluster velocities from measurements of the kinematic Sunyaev-Zeldovich effect, Astrophys. J. 372 (1991) 21.

[185] D.A. Riechers et al., A Dust-Obscured Massive Maximum-Starburst Galaxy at a Redshift of 6.34, Nature 496 (2013) 329 [arXiv:1304.4256] [INSPIRE].

[186] E. Rozo, E.S. Rykoff, J.G. Bartlett and A.E. Evrard, Cluster Cosmology at a Crossroads: Neutrino Masses, arXiv:1302.5086 [INSPIRE].

[187] J.A. Rubiño-Martín, J. Chluba and R.A. Sunyaev, Lines in the cosmic microwave background spectrum from the epoch of cosmological hydrogen recombination Mon. Not. R. Astron. Soc. 371 (2006) 1939.

[188] J.A. Rubiño-Martín, J. Chluba and R.A. Sunyaev, Lines in the cosmic microwave background spectrum from the epoch of cosmological helium recombination, Astron. Astrophys. 485 (2008) 377.

[189] J.A. Rubiño-Martín, C. Hernández-Monteagudo and R.A. Sunyaev, The imprint of cosmological hydrogen recombination lines on the power spectrum of the CMB, Astron. Astrophys. 438 (2005) 461.

[190] E.S. Rykoff et al., The $L_{X}-M$ relation of clusters of galaxies, Mon. Not. R. Astron. Soc. 387 (2008) L28.

[191] S. Sazonov and R. Sunyaev, CMB radiation in the direction of a moving cluster of galaxies with hot gas: Relativistic corrections, Astrophys. J. 508 (1998) 1 [astro-ph/9804125] [INSPIRE].

[192] U. Seljak and M. Zaldarriaga, Lensing induced cluster signatures in cosmic microwave background, Astrophys. J. 538 (2000) 57 [astro-ph/9907254] [INSPIRE].

[193] B.D. Sherwin et al., The Atacama Cosmology Telescope: Cross-Correlation of CMB Lensing and Quasars, Phys. Rev. D 86 (2012) 083006 [arXiv:1207.4543] [INSPIRE].

[194] J. Smidt, A. Amblard, C.T. Byrnes, A. Cooray, A. Heavens and D. Munshi, CMB Constraints on Primordial non-Gaussianity from the Bispectrum $\left(f_{N L}\right)$ and Trispectrum $\left(g_{N L}\right.$ and $\left.\tau_{N L}\right)$ and a New Consistency Test of Single-Field Inflation, Phys. Rev. D 81 (2010) 123007 [arXiv: 1004.1409] [INSPIRE].

[195] K.M. Smith, O. Zahn and O. Dore, Detection of Gravitational Lensing in the Cosmic Microwave Background, Phys. Rev. D 76 (2007) 043510 [arXiv:0705.3980] [INSPIRE].

[196] K.M. Smith et al., CMBPol Mission Concept Study: Gravitational Lensing, AIP Conf. Proc. 1141 (2009) 121.

[197] G.F. Smoot et al., Structure in the COBE differential microwave radiometer first year maps, Astrophys. J. 396 (1992) L1 [INSPIRE].

[198] S.-C. Su, E.A. Lim and E. Shellard, CMB Bispectrum from Non-linear Effects during Recombination, arXiv:1212.6968 [INSPIRE].

[199] R.A. Sunyaev and J. Chluba, Signals from the epoch of cosmological recombination (Karl Schwarzschild Award Lecture 2008), Astronomische Nachrichten 330 (2009) 657.

[200] R.A. Sunyaev and Y.B. Zeldovich, Small scale entropy and adiabatic density perturbations Antimatter in the Universe, Asptrophys. Space Sci. 9 (1970) 368.

[201] R. Sunyaev and Y. Zeldovich, Formation of clusters of galaxies: Protocluster fragmentation and intergalactic gas heating, Astron. Astrophys. 20 (1972) 189 [INSPIRE].

[202] R.A. Sunyaev and Y.B. Zeldovich, The Observations of Relic Radiation as a Test of the Nature of X-Ray Radiation from the Clusters of Galaxies, Comments on Astrophysics and Space Physics 4 (1972) 173. 
[203] H. Tashiro, E. Sabancilar and T. Vachaspati, CMB Distortions from Superconducting Cosmic Strings, Phys. Rev. D 85 (2012) 103522 [arXiv:1202.2474] [INSPIRE].

[204] H. Tashiro, E. Sabancilar and T. Vachaspati, CMB Distortions from Damping of Acoustic Waves Produced by Cosmic Strings, JCAP 08 (2013) 035 [arXiv:1212.3283] [INSPIRE].

[205] H. Tashiro, J. Silk and D.J.E. Marsh, Constraints on primordial magnetic fields from CMB distortions in the axiverse, Phys. Rev. D 88 (2013) 125024 [arXiv:1308.0314] [INSPIRE].

[206] Planck HFI Core Team, Planck early results. IV. First assessment of the High Frequency Instrument in-flight performance, Astron. Astrophys. 536 (2011) A4.

[207] T. Treu, Strong Lensing by Galaxies, Annu. Rev. Astron. Astr. 48 (2010) 87.

[208] A. Vallinotto, The synergy between the Dark Energy Survey and the South Pole Telescope, Astrophys. J. 778 (2013) 108 [arXiv:1304.3474] [INSPIRE].

[209] A. Vallinotto, S. Das, D.N. Spergel and M. Viel, Lenses in the forest: cross-correlation of the Lyman-alpha flux with CMB lensing, Phys. Rev. Lett. 103 (2009) 091304 [arXiv:0903.4171] [INSPIRE].

[210] C. van de Bruck, J. Morrice and S. Vu, Constraints on Disformal Couplings from the Properties of the Cosmic Microwave Background Radiation, arXiv:1303.1773 [INSPIRE].

[211] A. van Engelen et al., A measurement of gravitational lensing of the microwave background using South Pole Telescope data, Astrophys. J. 756 (2012) 142 [arXiv:1202.0546] [INSPIRE].

[212] L. Verde, H. Peiris and R. Jimenez, Optimizing CMB polarization experiments to constrain inflationary physics, JCAP 01 (2006) 019 [astro-ph/0506036] [INSPIRE].

[213] M. Viero et al., HerMES: Cosmic Infrared Background Anisotropies and the Clustering of Dusty Star-Forming Galaxies, Astrophys. J. 772 (2013) 77 [arXiv: 1208.5049] [INSPIRE].

[214] L. Wang et al., HerMES: detection of cosmic magnification of submillimetre galaxies using angular cross-correlation, Mon. Not. R. Astron. Soc. 414 (2011) 596.

[215] J.-Q. Xia, M. Negrello, A. Lapi, G. De Zotti, L. Danese and M. Viel, Clustering of submillimetre galaxies in a self-regulated baryon collapse model, Mon. Not. R. Astron. Soc. 422 (2012) 1324.

[216] Q.-J. Yu, D.N. Spergel and J.P. Ostriker, Rayleigh scattering and microwave background fluctuations, Astrophys. J. 558 (2001) 23 [astro-ph/0103149] [INSPIRE].

[217] M. Zaldarriaga and U. Seljak, Reconstructing projected matter density from cosmic microwave background, Phys. Rev. D 59 (1999) 123507 [astro-ph/9810257] [INSPIRE].

[218] Y.B. Zeldovich, V.G. Kurt and R.A. Syunyaev, Recombination of Hydrogen in the Hot Model of the Universe, Zh. Eksp. Teor. Fiz. 55 (1968) 278.

[219] Y.B. Zeldovich and R.A. Sunyaev, The Interaction of Matter and Radiation in a Hot-Model Universe, Asptrophys. Space Sci. 4 (1969) 301.

[220] P. Zhang, U.-L. Pen and H. Trac, The temperature of the intergalactic medium and the Compton y parameter, Mon. Not. R. Astron. Soc. 355 (2004) 451. 


\section{The PRISM collaboration}

Philippe André, ${ }^{1}$ Carlo Baccigalupi, ${ }^{2}$ Anthony Banday, ${ }^{3,4}$ Domingos Barbosa,${ }^{5}$ Belen Barreiro, ${ }^{6}$ James Bartlett, ${ }^{7,8}$ Nicola Bartolo, ${ }^{9,10}$ Elia Battistelli, ${ }^{11}$ Richard Battye, ${ }^{12}$ George Bendo, ${ }^{13}$ Alain Benoît, ${ }^{14}$ Jean-Philippe Bernard, ${ }^{4}$ Marco Bersanelli, ${ }^{16,17}$ Matthieu Béthermin, ${ }^{18}$ Pawel Bielewicz, ${ }^{2}$ Anna Bonaldi, ${ }^{12}$ Fran, cois Bouchet, ${ }^{19,20}$ Fran, cois Boulanger, ${ }^{21}$ Jan Brand, ${ }^{22,23}$ Martin Bucher, ${ }^{7}$ Carlo Burigana, ${ }^{23,24}$ Zhen-Yi Cai, ${ }^{2,25,26}$ Philippe Camus, ${ }^{15}$ Francisco Casas, ${ }^{6}$ Viviana Casasola, ${ }^{23}$ Guillaume Castex,${ }^{7}$ Anthony Challinor, ${ }^{19,30,31}$ Jens Chluba, ${ }^{32}$ Gayoung Chon, ${ }^{33}$ Sergio Colafrancesco, ${ }^{34}$ Barbara Comis, ${ }^{35}$ Francesco Cuttaia, ${ }^{23}$ Giuseppe D'Alessandro, ${ }^{11}$ Antonio Da Silva, ${ }^{36}$ Richard Davis, ${ }^{12}$ Miguel de Avillez, ${ }^{37,38}$ Paolo de Bernardis, ${ }^{11}$ Marco de Petris, ${ }^{11}$ Adriano de Rosa, ${ }^{23}$ Gianfranco de Zotti, ${ }^{39,2}$ Jacques Delabrouille, ${ }^{7}$ Fran, cois-Xavier Désert, ${ }^{40}$ Clive Dickinson, ${ }^{12}$ Jose Maria Diego, ${ }^{6}$ Joanna Dunkley, ${ }^{41}$ Torsten Enßlin, ${ }^{42}$ Josquin Errard ${ }^{43}$ Edith Falgarone, ${ }^{44}$

Pedro Ferreira, ${ }^{41}$ Katia Ferrière, ${ }^{4}$ Fabio Finelli, ${ }^{23,45}$ Andrew Fletcher, ${ }^{46}$ Pablo Fosalba, ${ }^{47}$ Gary Fuller ${ }^{12}$ Silvia Galli, ${ }^{19,20}$ Ken Ganga, ${ }^{7}$ Juan García-Bellido, ${ }^{48}$ Adnan Ghribi, ${ }^{7}$ Martin Giard, ${ }^{3,4}$ Yannick Giraud-Héraud, ${ }^{7}$ Joaquin Gonzalez-Nuevo, ${ }^{6,2}$ Keith Grainge, ${ }^{12}$ Alessandro Gruppuso, ${ }^{23}$ Alex Hall, ${ }^{49}$ Jean-Christophe Hamilton, ${ }^{7}$ Marijke Haverkorn, ${ }^{50,51}$ Carlos Hernandez-Monteagudo, ${ }^{52}$ Diego Herranz, ${ }^{6}$ Mark Jackson, ${ }^{7}$ Andrew Jaffe, ${ }^{53}$ Rishi Khatri, ${ }^{42}$ Martin Kunz, ${ }^{54,55}$ Luca Lamagna, ${ }^{11}$ Massimiliano Lattanzi, ${ }^{24,22}$ Paddy Leahy, ${ }^{12}$ Julien Lesgourgues, ${ }^{56,57,58}$ Michele Liguori, ${ }^{9}$ Elisabetta Liuzzo, ${ }^{23}$ Marcos Lopez-Caniego, ${ }^{6}$ Juan Macias-Perez, ${ }^{35}$ Bruno Maffei, ${ }^{12}$ Davide Maino, ${ }^{16}$ Anna Mangilli, ${ }^{19}$ Enrique Martinez-Gonzalez, ${ }^{6}$ Carlos J.A.P. Martins, ${ }^{60}$ Silvia Masi, ${ }^{11}$ Marcella Massardi, ${ }^{23}$ Sabino Matarrese, ${ }^{9,10}$ Alessandro Melchiorri, ${ }^{11,59}$ Jean-Baptiste Melin, ${ }^{60}$ Aniello Mennella, ${ }^{16}$ Arturo Mignano, ${ }^{23}$

Marc-Antoine Miville-Deschênes, ${ }^{21}$ Alessandro Monfardini, ${ }^{15}$ Anthony Murphy, ${ }^{62}$ Pavel Naselsky, ${ }^{63,64}$ Federico Nati, ${ }^{11}$ Paolo Natoli, ${ }^{24,22,23,65}$ Mattia Negrello, ${ }^{39}$ Fabio Noviello, ${ }^{12}$ Créidhe O'Sullivan, ${ }^{62}$ Francesco Paci, ${ }^{2}$ Luca Pagano, ${ }^{11,59}$ Rosita Paladino, ${ }^{23,28}$ Nathalie Palanque-Delabrouille, ${ }^{61}$ Daniela Paoletti, ${ }^{23,17}$ Hiranya Peiris,${ }^{66}$ Francesca Perrotta,${ }^{2}$ Francesco Piacentini, ${ }^{11}$ Michel Piat, ${ }^{7}$ Lucio Piccirillo, ${ }^{13,67}$ Giampaolo Pisano, ${ }^{12,68}$ Gianluca Polenta, ${ }^{69,70}$ Agnieszka Pollo, ${ }^{71,72}$ Nicolas Ponthieu, ${ }^{15}$ Mathieu Remazeilles, ${ }^{12,7}$ Sara Ricciardi, ${ }^{23}$ Matthieu Roman, ${ }^{7}$ Cyrille Rosset, ${ }^{7}$ Jose-Alberto Rubino-Martin, ${ }^{73,74}$ Maria Salatino, ${ }^{11}$

Alessandro Schillaci, ${ }^{11}$ Paul Shellard, ${ }^{31}$ Joseph Silk, ${ }^{19,32,41}$ Alexei Starobinsky, ${ }^{75}$ Radek Stompor, ${ }^{7}$ Rashid Sunyaev, ${ }^{42}$ Andrea Tartari, ${ }^{7}$ Luca Terenzi, ${ }^{23}$ Luigi Toffolatti, ${ }^{76,6}$ Maurizio Tomasi, ${ }^{16,17}$ Neil Trappe, ${ }^{62}$ Matthieu Tristram, ${ }^{77}$ Tiziana Trombetti, ${ }^{23}$ Marco Tucci, ${ }^{54}$ Rien Van de Weijgaert, ${ }^{78}$ Bartjan Van Tent, ${ }^{79}$ Licia Verde, ${ }^{80,81}$ Patricio Vielva, ${ }^{6}$ Ben Wandelt, ${ }^{19,82}$ Robert Watson, ${ }^{12}$ Stafford Withington, ${ }^{83}$

1 Laboratoire d'Astrophysique de Paris-Saclay, Gif-sur-Yvette Cedex, France

2 SISSA, Via Bonomea 265, 34136, Trieste, Italy

3 Université de Toulouse, UPS-OMP, IRAP, F-31028 Toulouse cedex 4, France

4 CNRS, IRAP, 9 Av. colonel Roche, BP 44346, F-31028 Toulouse cedex 4, France

${ }^{5}$ Grupo de Radio Astronomia Basic Sciences 8 Enabling Technologies Instituto de

Telecomunicações, Campus Universitario de Santiago, 3810-193 Aveiro, Portugal

6 Instituto de Fisica de Cantabria (CSIC-Universidad de Cantabria) Avda. de los Castros s/n, 39005 Santander, Spain 
7 APC, AstroParticule et Cosmologie, Université Paris Diderot, CNRS/IN2P3, CEA/lrfu,

Observatoire de Paris, Sorbonne Paris, Cité, 10, rue Alice Domon et Léonie Duquet, 75205 Paris

Cedex 13, France

8 Jet Propulsion Laboratory, California Institute of Technology, 4800 Oak Grove Drive, Pasadena, California, U.S.A.

9 Dipartimento di Fisica e Astronomia "G. Galilei", Università degli studi di Padova, via Marzolo

8, I-35131, Padova, Italy

10 INFN, Sezione di Padova, via Marzolo 8, I-35131, Padova, Italy

11 Dipartimento di Fisica, Università di Roma La Sapienza, P.le A. Moro 2, 00185 Roma, Italy

12 Jordell Bank Centre for Astrophysics, School of Physics $\&$ Astronomy, University of Manchester,

Oxford Road, Manchester M13 9PL, U.K.

13 U.K. ALMA Regional Centre Node, Jordell Bank Centre for Astrophysics, School of Physics and Astronomy, University of Manchester, Oxford Road, Manchester M13 9PL, United Kingdom

14 Physics 85 Astronomy, University of Manchester, Oxford Road, Manchester M13 9PL, U.K.

15 Institut Néel, CNRS, Université Joseph Fourier Grenoble I, 25 rue des Martyrs, Grenoble, France

16 Dipartimento di Fisica, Università degli Studi di Milano, Via Celoria, 16, Milano, Italy

17 INAF/IASF Milano, Via E. Bassini 15, Milano, Italy

18 European Southern Observatory, Karl-Schwarzschild-Str. 2, 85748 Garching, Germany

19 Institut d'Astrophysique de Paris, CNRS (UMR7095), 98 bis Boulevard Arago, F-75014, Paris, France

20 UPMC Univ Paris 06, UMR7095, 98 bis Boulevard Arago, F-75014, Paris, France

21 Institut d'Astrophysique Spatiale, CNRS (UMR8617) Université Paris-Sud 11, Bâtiment 121, Orsay, France

22 INFN, Sezione di Ferrara, Ferrara, Italy

23 INAF - Istituto di Astrofisica Spaziale e Fisica Cosmica, via Gobetti 101, 40129, Bologna, Italy

24 Dipartimento di Fisica e Scienze della Terra, Università di Ferrara, Via Saragat 1, 44122

Ferrara, Italy

25 Department of Astronomy, Xiamen University, Xiamen 361005, China

${ }^{26}$ Center for Astrophysics, University of Science and Technology of China, Hefei 230026, China

27 INAF - Osservatorio Astronomico di Arcetri, Largo Enrico Fermi 5, 50125 Firenze, Italy

28 Dipartimento di Fisica e Astronomia, Università di Bologna, Viale Berti Pichat 6/2, 40127,

Bologna, Italy

29 Institute of Astronomy, University of Cambridge, Madingley Road, Cambridge CBз 0HA, U.K.

${ }^{30}$ Kavli Institute for Cosmology Cambridge, Madingley Road,Cambridge, CB3 OHA, U.K.

31 Centre for Theoretical Cosmology, DAMTP, University of Cambridge, Wilberforce Road, Cambridge CB3 0WA U.K.

32 Johns Hopkins University, Bloomberg Center 435, 3400 N Charles St., Baltimore, 21231 MD, U.S.A.

${ }^{33}$ Max-Planck-Institut Für Extraterrestriche Physik, D-85748 Garching, Germany

34 School of Physics, University of the Witwatersrand, 1 Jan Smuts Avenue, Braamfontein, Johannesburg, 2050 South Africa

35 Laboratoire de Physique Subatomique et de Cosmologie, Université Joseph Fourier Grenoble I, CNRS/IN2P3, Institut National Polytechnique de Grenoble, 53 rue des Martyrs, 38026 Grenoble cedex, France

${ }^{36}$ Centro de Astrofísica, Universidade do Porto, Rua das Estrelas,4149-761 Porto, Portugal

37 Department of Mathematics, University of Evora, R. Romao Ramalho 58, 6999-670 Evora,

Portugal

38 Zentrum für Astronomie und Astrophysik, Technische Universität Berlin, Hardenbergstr. 36,

D-10622 Berlin, Germany

39 INAF - Osservatorio Astronomico di Padova, Vicolo dell'Osservatorio 5, Padova, Italy

40 IPAG: Institut de Planétologie et d'Astrophysique de Grenoble, Université Joseph Fourier,

Grenoble 1 / CNRS-INSU, UMR 5272, Grenoble, F-38039, France

41 Astrophysics, University of Oxford, Keble Road, Oxford OX1 3RH, U.K. 
42 Max-Planck-Institut für Astrophysik, Karl-Schwarzschild-Str. 1, 85739 Garching, Germany

43 Lawrence Berkeley National Laboratory 1, Cyclotron Rd, MS 48R4047 Berkeley, CA 94718, 8151

U.S.A.

${ }^{44}$ LERMA, CNRS, Observatoire de Paris, 59 Avenue de l'Observatoire, Paris, France

45 INFN, Sezione di Bologna, Via Irnerio 44, I-40124, Bologna, Italy

46 Newcastle University, U.K.

47 Institut de Ciencies de L'Espai (IEEC-CSIC), Facultat de Ciencies, Campus UAB, Torre C5, par 2 a planta, 8191 Cerdanyola (Barcelona), Spain

48 Instituto de Fisica Teorica CSIC-UAM, c/ Nicolas Cabrera, 13 Universidad Autonoma de Madrid, Cantoblanco 28047, Madrid, Spain

49 Institute for Astronomy, University of Edinburgh, Royal Observatory, Blackford Hill, Edinburgh, EH9 3HJ, U.K.

${ }^{50}$ Department of Astrophysics/IMAPP, Radboud University Nijmegen, P.O. Box 9008, 6498 GL

Nijmegen, The Netherlands

51 Leiden Observatory, Leiden University, P.O. Box 9511, 2298 RA Leiden, The Netherlands

52 Centro de Estudios de Fisica del Cosmos de Aragon (CEFCA) Plaza San Juan 1, planta 2,

E-43999 Teruel, Spain

53 Blackett Laboratory, Imperial College, Prince Consort Road, London SWr 2AZ U.K.

54 Département de Physique Théorique and Center for Astroparticle Physics, Université de Genève, 24 quai Ernest Ansermet, CH-1209 Genève 4, Switzerland

55 African Institute for Mathematical Sciences, 6 Melrose Road, Muizenberg, 7943, South Africa

56 ITP, Ècole Polytechnique Fèdèrale de Lausanne, CH-1013 Lausanne, Switzerland

57 CERN, Theory Division, CH-1209 Geneva 23, Switzerland

58 LAPTh, Universitéde Savoie and CNRS, BP108, 74939 Annecy-le-Vieux Cedex, France

59 INFN, Sezione di Roma 1, Università di Roma Sapienza, Piazzale Aldo Moro 2, 183, Roma, Italy

60 CAUP, Rua das Estrelas s/n, 4148-760 Porto, Portugal

61 DSM/Irfu/SPP, CEA-Saclay, F-91189 Gif-sur-Yvette Cedex, France

62 Department of Experimental Physics, National University of Ireland, Maynooth, Ireland

63 Niels Bohr Institute, Blegdamsvej 17, Copenhagen, Denmark

64 Discovery Center for particle physics and cosmology, Blegdamsvej, 19, Copenhagen, Denmark

65 Agenzia Spaziale Italiana Science Data Center, c/o ESRIN, via Galileo Galilei, 42 Frascati, Italy

66 Department of Physics and Astronomy, University College London, Gower Street, London WC1E 6BT, United Kingdom

67 Photon Science Institute University of Manchester, U.K.

68 Cardiff University, U.K.

69 ASI Science Data Center, via del Politecnico snc, 130, Roma, Italy

70 INAF - Osservatorio Astronomico di Roma, via di Frascati 33, 39, Monte Porzio Catone, Italy

71 Astronomical Observatory of the Jagiellonian Universityul. Orla 168, 30-241 Kraków, Poland

72 National Centre for Nuclear Research (NCBJ), ul. Hoża 66 00-678 Warszawa, Poland

73 Instituto de Astrofisica de Canarias, E-38197 La Laguna, Tenerife, Spain

74 Departamento de Astrofisica, Universidad de La Laguna, E-38203 La Laguna, Tenerife, Spain

75 L.D. Landau Institute for Theoretical Physics RAS, Moscow, 119331, Russia

76 Department of Physics, University of Oviedo, avda. calvo Sotelo s/n, 33004 Oviedo, Spain

77 LAL, Université Paris-Sud, CNRS/IN2P3, Orsay, France

78 Kapteyn Astronomical Institute, University of Groningen, P.O. Box 797, 9744 AV Groningen, The Netherlands

${ }^{79}$ Laboratoire de Physique Théorique, Université Paris-Sud 11 and CNRS, Bâtiment 20\%, 91402 Orsay Cedex, France

${ }^{80}$ ICREA $\&$ Instituto de Ciencias del Cosmos (ICC-UB IEEC) Universidad de Barcelona Marti $i$ Franques 1, 8025-E Barcelona, Spain

81 Institute of Theoretical Astrophysics, University of Oslo, P.O. Box 1026 Blindern, N-312 Oslo, Norway 
${ }^{82}$ Institut Lagrange de Paris (ILP), Sorbonne Universités, 95bis boulevard Arago, F-75011 Paris, France

${ }^{83}$ University of Cambridge, Cavendish Laboratory, J.J. Thomson Avenue, Cambridge CB3 OHE, U.K.

\section{The PRISM Science Case and White Paper Coordination}

The preparation of the science case submitted to ESA has been coordinated by: James Bartlett, François Bouchet, François Boulanger, Martin Bucher, Anthony Challinor, Jens Chluba, Paolo de Bernardis (spokesperson), Gianfranco de Zotti, Jacques Delabrouille (coordinator), Pedro Ferreira, Bruno Maffei

The writing of the extended version of the PRISM white paper has been coordinated by:

Martin Bucher, Jacques Delabrouille

\section{The PRISM Steering Committee}

France: François Bouchet, Martin Bucher, Jacques Delabrouille, Martin Giard Germany: Jens Chluba, Rashid Sunyaev

Ireland: Anthony Murphy

Italy: Marco Bersanelli, Carlo Burigana, Paolo de Bernardis

Netherlands: Rien van de Weijgaert

Portugal: Carlos Martins

Spain: Enrique Martínez-González, José Alberto Rubiño-Martín, Licia Verde

Switzerland: Martin Kunz

United Kingdom: Anthony Challinor, Joanna Dunkley, Bruno Maffei 